\title{
Self-Sufficiency Incomes and Increasing Precarity in South Dakota's Job Market, 2006-2015
}

\author{
Karen M. Al-Ahmin, Doctoral Candidate \\ Argosy University Twin Cities, Eagan, MN
}

This longitudinal study examined the labor market of South Dakota, a state with an exceptionally low unemployment rate, over the ten years spanning 2006 to 2015. Analysis focused on the questions, what proportion of the market is made up of low wage jobs? and are the numbers of low wage workers increasing or decreasing over time? Data collected by the U. S. Census Bureau and U. S. Department of Commerce's Bureau of Labor Statistics (BLS) was dissected on a micro level to determine which occupations held disproportionate numbers of low paying jobs. A threshold for households to live free of subsidies was set, then compared to workers' incomes. Detailed data mining revealed information that contributes to the literature of employment and labor fields, strategic economic development, and the role of public policy in helping citizens get and stay independent of subsidies.

\section{PROBLEM BACKGROUND}

There is considerable disagreement about the current state of domestic labor markets and the status of the American worker. Blue collar factory work that once supported America's manufacturing base is now considered part of a bygone era (Forrant, 2002). The current domestic labor market rarely offers high school graduates the ability to secure unionized work offering middle-class wages, job security, pension, and healthcare benefits (Figueroa, Grabelsky, \& Lamare, 2011). Further exacerbated by the financial collapse of 2008, free market globalization produced a preponderance of precarious jobs (Kalleberg, 2009) while simultaneously eroding the middle-class (Boushey, Fremstad, Gragg, \& Waller, 2007).

Despite this changed landscape, immigrants worldwide still look to America as the land of opportunity (Stoll, 2009). For those living here, however, the American Dream may be a little less attainable than the ideal would suggest. Kalleberg (2009) defined precarious work as any "employment that is uncertain, unpredictable, and risky from the point of view of the worker" (p. 2). European researcher, Olsthoorn (2014), described precariousness as the intersection of three components: (a) insecure employment, such as fixed-term contracts and temporary agency work; (b) unsupportive entitlements, particularly during unemployment periods; and (c) vulnerable employees, those without other means of subsistence or a partner with a significant income. Figure 1 reproduces Olsthoorn's (2014) conceptual framework. The author summarized "precarious employment as the linking of vulnerable workers to insecure jobs and unsupportive [employment relation] entitlements" (p. 424). 


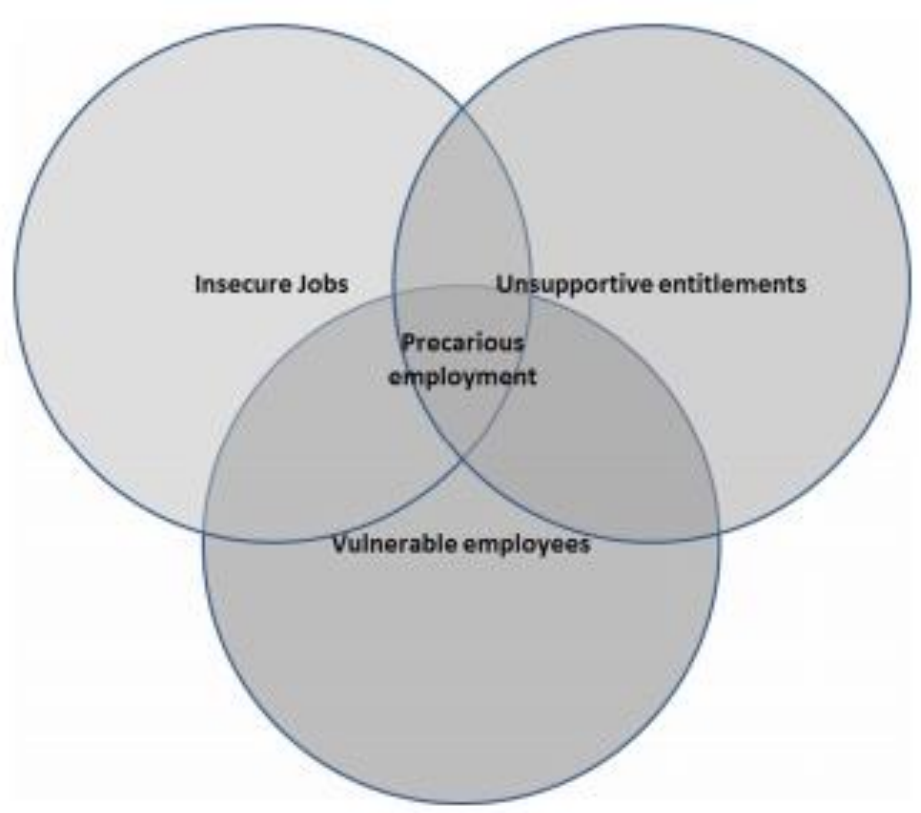

Figure.1. Conceptual framework showing precarious employment as the intersection of vulnerable employees in insecure jobs with unsupportive entitlements.

Reference: Olsthoorn, M. (2014). Measuring precarious employment: A proposal for two indicators of precarious employment based on set-theory and tested with Dutch labor market data. Social Indicators Research: An International and Interdisciplinary Journal for Quality-of-Life Measurement, 119(1), 421-441. doi:10.1007/s11205-013-0480-y. Reprinted with permission of Springer.

\section{Turnover}

Some economic indicators, like high turnover rates, do not fully convey the negative effects for workers. Generally, high turnover rates are considered a sign of a good economy (Gustafson, 2014). When skilled workers can freely transfer jobs to more lucrative positions or shift to contract work that would pay higher project rates than could be earned as a permanent employee of an organization, turnover can be beneficial. On the other end of the spectrum, however, low paying jobs with high turnover rates result in far different effects for workers.

Jobs with the lowest pay also often require minimal qualifications. With little to differentiate one job from another, they become interchangeable - not only what workers think of jobs, but what employers think of workers. To illustrate, part-time retail experienced an average 74.9\% turnover rate in 2013 (Gustafson, 2014). Where employees did receive ongoing training, however, a two-year study found sales performance increases of $69 \%$ to $123 \%$ compared to less engaged retail personnel (Netessine, 2014). Despite the significant revenue boosting benefit to retaining employees, employers continue to offer part-time work at or near minimum hourly wages (Netessine, 2014). 
These employers simultaneously complain about the costs and effort associated with high turnover rates, yet continue to offer the kinds of low paying precarious jobs that nearly guarantee such turnover. In fact, over the last two decades, low skilled jobs have not experienced significant wage increases beyond federally mandated minimum wage raises (Brown, 2016). Employers offering these low wage positions do so with little advancement opportunities, and no regular or substantial hourly wage increases to encourage loyalty in workers (Netessine, 2014).

One study showed nonwage compensation factors, like healthcare benefits, normally represented $28 \%$ of total compensation for U. S. private workers (Lettau \& Buchmueller, 1999); yet, part-time workers received few, if any, of these benefits. Researchers found, where healthcare benefits were offered, for example, they were structured so that part-time workers paid more for less coverage than their full-time coworkers (Lettau \& Buchmueller, 1999). The lack of equity between workers extends further into the stagnation of low wage positions when compared to more demanding jobs.

From 1990 to 2015, Brown (2016) found the average hourly wage for jobs with higher analytical and social skills increased by four dollars per hour. Jobs requiring greater physical skills rose two dollars per hour over the same period (Brown, 2016). With such dramatic discrepancies between increases for low wage work compared to more academically or physically demanding jobs, some researchers have called for interventions to make things more equitable (Boushey et al., 2007; Kalleberg, 2009).

Boushey et al. (2007) argued that chronic low wage work threatens national prosperity in the long-term by oppressing whole sectors of the population. What Olsthoorn (2014) called polarized labor markets, he explained as the result of "changing patterns of production and consumption, slower economic growth and substantive changes in social and economic policy in recent decades" (p. 422). Olsthoorn warned that those in low paying jobs with little job security are also at a "disproportionately high risk of adverse psychological and physiological effects and are in the disempowered segment of the dualized labor market" (p. 422).

An adverse psychological effect can be found in the interchangeable attitudes of both employers and employees. Lack of advancement opportunities can discourage whole segments of the population, forcing workers to trade one entry level job for another - further retarding career advancement (Netessine, 2014). To combat these unproductive attitudes and actions, Kalleberg (2009) believed reform would require legislators to enact initiatives targeted toward quality job growth. Through changes in public policy, voluntary turnover rates could be reduced "to tackle the causes and consequences of precarity and to rebuild the social contract" (p. 16).

As European nations attempt to address the issues of a growing precarious work force, America stands as an example of where temporary employment agencies get larger after each recession (Broughton et al., 2016). With more workers contractually employed, the insecure nature of precarity spreads into the skilled work force. European researchers offered suggestions to "combat marginal part-time work and encourage an increase in working hours for those that want to work more" (p. 173). These included raising entitlement thresholds and supplementing 
social security and pension benefits. The researchers emphasized that workers without substantial wages and without job security have difficulty planning, including retirement (Broughton et al., 2016).

\section{Subsidies}

An examination of South Dakota's labor force demonstrates a gap between being employed and being self-sufficient (Ruggles, Genadek, Goeken, Grover, \& Sobek, 2015). Although South Dakota has a nationally low unemployment rate, $2.9 \%$ in 2016, a considerable portion of available work does not reach the threshold of self-sufficiency in terms of wages, hours, or both (BLS, 2016b). Therefore, these families may end up turning to government programs for assistance.

Even though it is not a standard for independence, the federal poverty threshold assigns a value for the level of income required by households of differing compositions to meet their basic needs. This standard uses the U. S. Department of Agriculture's Thrifty food plan, intended "for temporary or emergency use when funds are low" (Eberstadt, 2006, p. 22). Despite being based on a value intended for temporary use, the federal poverty threshold routinely serves as the eligibility requirement for many federally funded programs. The most utilized of these programs include Medicaid, Supplemental Nutrition Assistance Program (SNAP), Temporary Assistance for Needy Families (TANF), subsidized housing, and school meal programs (HHS, 2016).

Each state administers these entitlements, but the programs are paid for by the federal government (Schott, Pavetti, \& Floyd, 2015). Unfortunately, restructuring these programs in 1996 into block grants for state discretionary use has not had the intended effect. In fact, the Center on Budget and Policy Priorities reported only $26 \%$ of TANF funds were paid out as aid to the needy by states in 2014 (Schott et al., 2015). A minimum of $41 \%$ of grant money involves funding for social workers, managers, upper administration, and "to fill state budget holes, and in some cases to substitute for existing state spending" (p. 1). Although each state's subsidy programs are funded by the federal government, each can choose to define eligibility qualifications differently.

In Minnesota, persons applying for free Medicaid coverage need to make no more than $133 \%$ of the federal poverty level for their family size (Minnesota, 2017). For 2016, a single person with no children could qualify for healthcare with an income up to $\$ 1,315$ per month ( $\$ 15,800$ per year); a household of four could earn up to $\$ 2,699$ per month ( $\$ 32,398$ per year) in income. If applicants exceed these thresholds, Minnesota has healthcare options for people earning up to $400 \%$ of the poverty level (Minnesota, 2017).

In South Dakota, however, Medicaid requires income levels of extreme poverty to qualify (HHS, 2016). In 2016, a household of one could not have a gross income greater than $\$ 613$ per month (\$7,356 per year); a household of four was limited to $\$ 986$ per month $(\$ 11,832$ per year). 
South Dakota sets Medicaid limitations to approximately $48 \%$ of the federal poverty guideline (Medicaid-help, 2017). Information from the U. S. Census Bureau's American Community Survey (ACS) for 2011-2015 indicated 14.48\% (119,501) of South Dakota's 825,506 population still met this extreme poverty requirement to receive Medicaid benefits. Additionally, children represented $25 \%(206,488)$ of the general population, but a disproportionate $33.19 \%(68,524)$ of all children received Medicaid (U. S. Census, 2016a).

After reforms made in 1996, federal cash entitlements can only be relied upon temporarily (DSS, 2017). To reduce the occurrences of the stereotypical welfare mom, someone who stays on public assistance by having additional children every few years, each adult now has 60 months of lifetime TANF benefits regardless of new births into the household (DSS, 2017). TANF, also called welfare or cash assistance, exists for families with no cash income. South Dakota's TANF, however, requires adults in the household to secure work with a minimum of 30 hours per week to qualify (DSS, 2017). This work requirement is problematic in that many low wage jobs do not offer 30 hours per week, and working at minimum wage for the required hours would cancel any cash benefit received by the household.

A snapshot for January 2016 reported South Dakota paid out \$1,292,093 in TANF benefits that month to 3,027 families, averaging $\$ 426.86$ per family (DSS, 2017). One factor notable in the data was that five of South Dakota's 66 counties received $59 \%$ of all benefits. These counties represent Indian reservations and the two major metropolitan areas. Pine Ridge Indian Reservation spans across Oglala Lakota and Pennington counties. Pennington also includes the Rapid City metropolitan area. Todd County houses the Rosebud Indian Reservation. Dewey County has the Cheyenne River Indian Reservation. Minnehaha County includes the Sioux Falls metropolitan area (DSS, 2017). These five counties represent both the poorest and most dense populations in the state. It is not surprising, therefore, these same areas also have the highest number of children qualified for free or reduced meals at school (SD Kids Count, 2017).

Figure 2 shows the percentage of children eligible to receive free or reduced breakfast and lunch for the school years 2001-2002 to 2015-2016 (SD Kids Count, 2017). Prior to the financial collapse of September 2008, the number of children qualified for the meal program consistently fell in the upper 30th percentile. With an immediate unemployment spike in 2008, participation crossed into the 40th percentile. Curiously, despite multiple years of recovery to end the Great Recession, low unemployment rates have not returned South Dakota's qualifying children below the 40th percentile (SD Kids Count, 2017). 


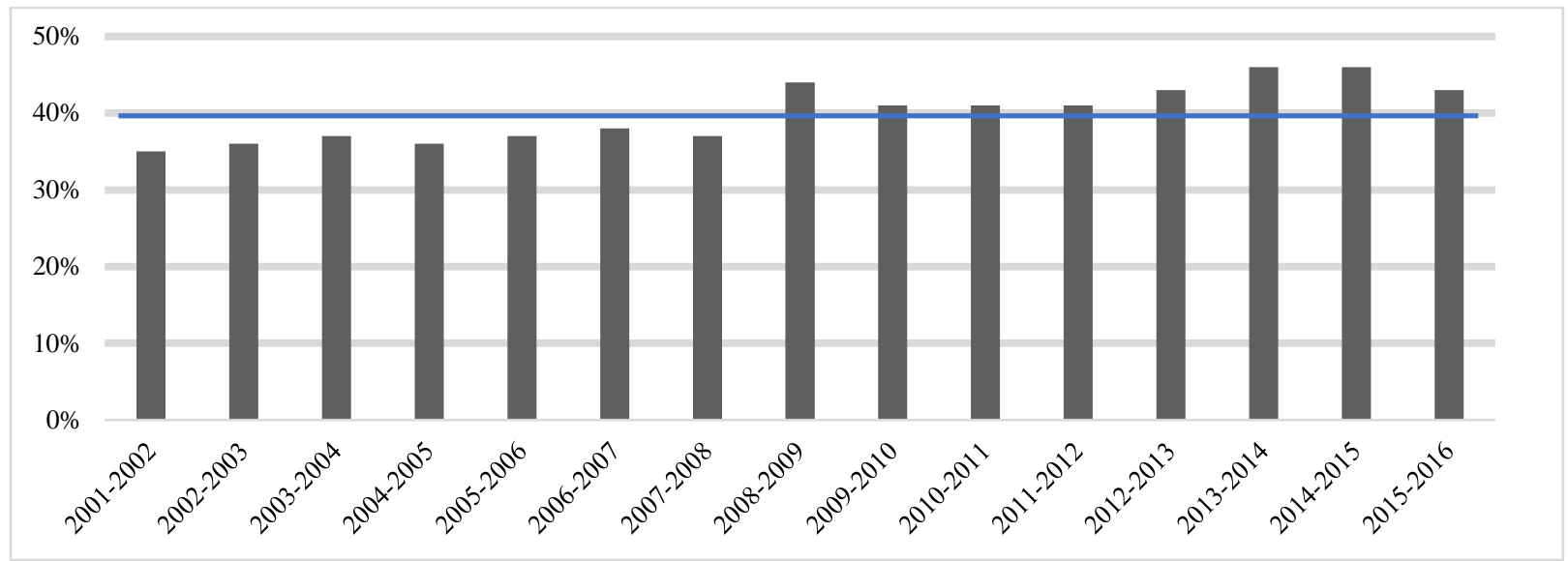

Figure 2. South Dakota children qualified for school meal programs 2001 to 2016. Adapted from South Dakota Kids Count. (2017, February 15), Child and Adult Nutrition Services, South Dakota Department of Education. Raw data retrieved from http://doe.sd.gov/cans/nslp.asp.

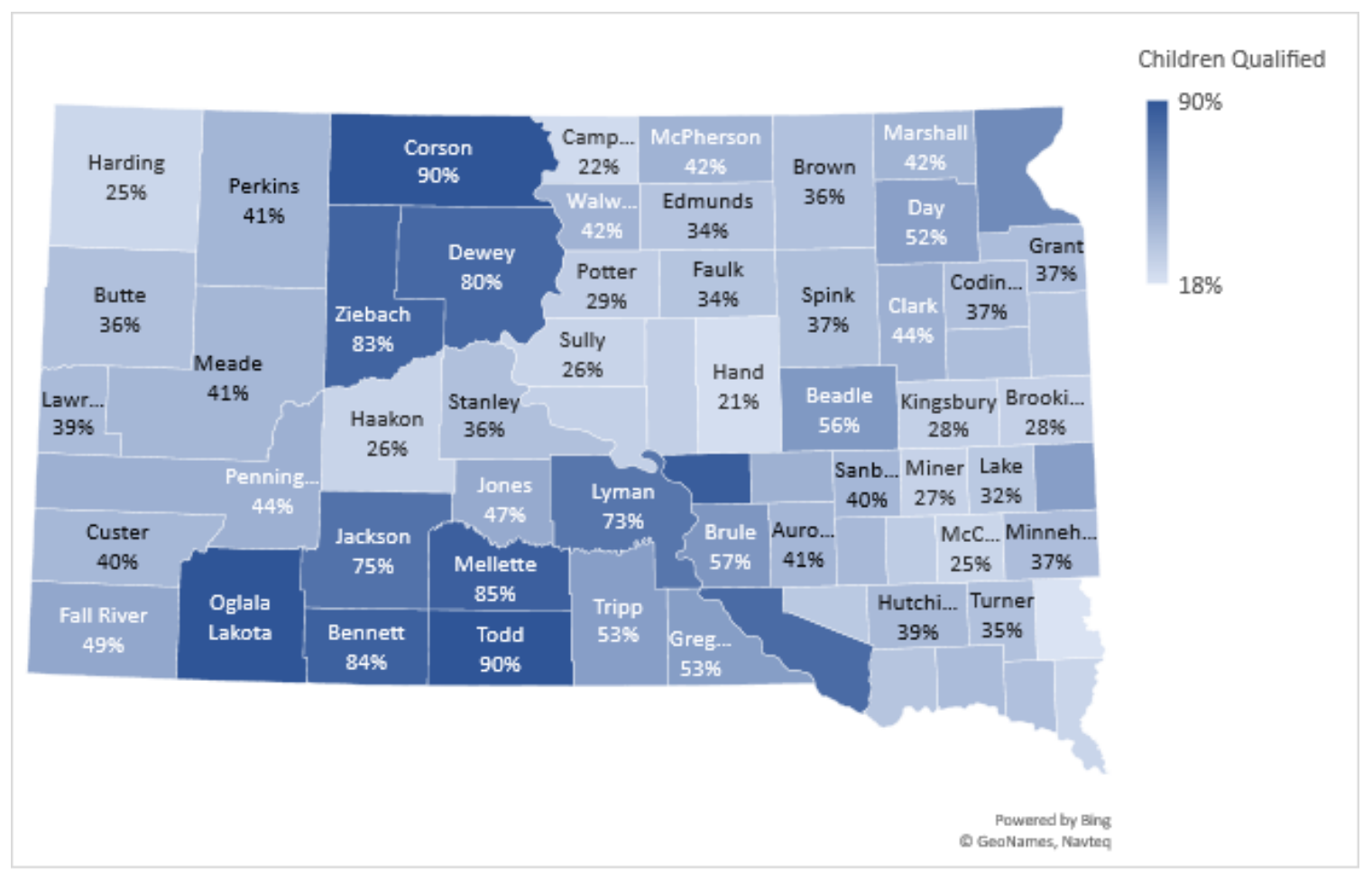

Figure 3. Children qualified for meal programs, by county, for 2015-2016 school year. Adapted from South Dakota Kids Count. (2017, February 15), Child and Adult Nutrition Services, South Dakota Department of Education. Raw data retrieved from http://doe.sd.gov/cans/nslp.asp. 
Figure 3 indicates the concentration of qualified children by county; this map closely mirrors the dispersion of TANF benefits discussed for January 2016 (DSS, 2017). In the higher concentration areas, food service program data indicated participation as $90 \%$ and above, rather than exact numbers on the upper values. Figure 3 lists these simply as 90\% (SD Kids Count, 2017). Clearly, low unemployment is not synonymous with incomes offering independence from subsidies.

\section{LITERATURE REVIEW}

Precarity has been well documented as a major concern globally, although the term has not been used extensively in the United States (Choi \& Mattoni, 2010). Building on the basic definition Kalleberg (2009) offered, where precarity is any "employment that is uncertain, unpredictable, and risky from the point of view of the worker" (p. 2), Choi and Mattoni (2010) defined "precarious work as all those forms of atypical employment characterized by short-term contracts and lacking social protection" (p. 215). Olsthoorn (2014) summarized "precarious employment as the linking of vulnerable workers to insecure jobs and unsupportive [employment relation] entitlements" (p. 424). This section looks at examples of how precarity can be mitigated through international research, union participation, and a consideration of income disparity.

\section{International Research}

Global attention into precarious work has prompted unionization to pool otherwise disparate groups into a block with representation (Choi \& Mattoni, 2010). In Russia, Bernabé and Kolev (2005) studied the composition of jobless and working poor in the Kyrgyz region. Not surprisingly, the researchers determined that a disproportionate number of youth, women, and internal migrants had a greater likelihood of unemployment and job instability. Among those who were employed, nearly $80 \%$ were subsistence farmers making far below the poverty level (Bernabé \& Kolev, 2005).

Russia is an example of a country with an entire class of workers considered unregistered and discouraged (Bernabé \& Kolev, 2005). Discouraged unemployed are those unemployed or underemployed for greater than two years, and who have given up on the idea of getting a decent job. In keeping with the International Labour Organization's (ILO) definition of unemployment, national statistics routinely exclude the discouraged unemployed - giving those statistics lower values than would be accurate. Once unemployment benefits expire, the ILO considers that individual no longer ranks among the unemployed - although that person most likely still is (Bernabé \& Kolev, 2005). As described, similarities between Russian discouraged workers and American chronically underemployed become apparent.

With Bernabé and Kolev (205), the purpose of their study was identification of groups in need of legislative intervention. To revitalize the Kyrgyz region, the researchers believed better

DOI: 10.17605/OSF.IO/QF4HY 
informed leaders could enact reforms and programs intended to stimulate the economy by providing jobs with stability and livable wages for its citizenry (Bernabé \& Kolev, 2005). Their work showed the relevance of academic research as a mechanism for public policy reform.

Reform, however, can work for or against the precarious. As a means of lowering high unemployment rates in Italy, employment was deregulated through legislative reforms in the 1980s (Choi \& Mattoni, 2010). Choi and Mattoni reported employers in 2010 had 40 "fixed-term contract options available" (p. 215), making the Italian labor market one of the most flexible in the European Union (EU). Statistics from 2000 through 2007 (pre-global recession) indicated three to four million workers did not have stable, secure employment. At that time, part-time and fixed-term work represented $25 \%$ of the work force. Although the Italian market had a large percentage of precarious workers, the country had slightly better percentages than the contemporary EU15 average (Choi \& Mattoni, 2010).

Despite having work contracts, Choi and Mattoni (2010) found precarious workers in Italy still dealt with lower wages, worse working conditions, and greater instability than permanent workers. In response to the growing proportion of flexible work arrangements, "all three Italian union federations created special unions for atypical workers in 1998" (p. 217). Briefly, unionization followed three strategic goals: "(1) re-regulation through collective bargaining, (2) political and legal actions, and (3) organizing and shop-floor representation" (Choi \& Mattoni, 2010, p. 220).

By 2014, the EU had expanded from 15 to 28 . In a study on precarious employment trends and patterns in 2014, as much as $25 \%$ of all EU28 jobs were temporary, part-time, or some combination (Broughton et al., 2016). Only 59\% of all jobs were characterized as both full-time and permanent. Additionally, without job security, many freelancers (11\%) and self-employed with employees (4\%) experienced less than full-time earnings. Even though these independent working options do offer a greater level of autonomy than other forms of employment, they potentially increase the precarious work force to as much as $41 \%$ of all workers (Broughton et al., 2016).

Most directly impactful to America, Buchanan and Chaparro (2008) highlighted the function of legal advocacy groups like the North American Agreement on Labor Cooperation (NAALC) within the North American Free Trade Agreement (NAFTA) to aid precarious workers. NAALC may have little power to remedy situations of complaint regarding the working conditions and organizing rights of precarious workers, but it does offer a platform to make these complaints into public awareness vehicles. Each of the three countries within NAFTA (Mexico, United States, Canada) must abide by the labor laws of each other. Where there are violations, advocacy lawyers file complaints. For example, Mexican workers in the apple orchards of Idaho complained of wage and working conditions. Of interest is that migrant workers are really all that is discussed per NAFTA. The agreement, however, intends to protect workers through the right to organize and collectively bargain at a time when unionization within the United States, ironically, has eroded by $95 \%$ of its pre-WWII levels (Buchanan \& Chaparro, 2008).

DOI: 10.17605/OSF.IO/QF4HY 


\section{Union Participation}

Even though unions have become less influential as a representation of the American work force over the last five decades, their infrastructures can offer new options to public and private work projects (Figueroa et al., 2011). Figueroa et al. determined "coalitions of unions and community organizations have succeeded in advancing employment and career models for demographic groups that have been traditionally underrepresented in the construction industry" (p. 2). Community Work Force Agreements (CWAs) produced Project Labor Agreements (PLAs) with expressed provisions of "targeted hiring and career development" (p. 2). What the researchers found was a mechanism for creating a career path in populations otherwise unlikely to attain a middle-class status on their own (Figueroa et al., 2011).

As a practical option, CWAs offered low income, at-risk, minority, unemployed, women, and other disenfranchised populations a route to a middle-class career (Figueroa et al., 2011). Pre-apprenticeship programs in high schools and community colleges bring students' skills up to the level needed to enter formal apprenticeship programs offered by unions. This system represents a hybrid between all-union and all-employer on-the-job training. Figueroa et al. (2011) found multiple construction companies contributed to the apprenticeship program run by unions, so that no one company supplied its competition.

\section{Income Disparity}

Nobel Laureate Michael Spence with Sandile Hlatshwayo (2011), in a working paper for the Council on Foreign Relations, examined the U. S. economy as a grouping of tradable and nontradable industries. In general, with the advent of an open global economy and advanced technologies, many tradable jobs efficiently have shifted to points in the value supply chain with cheaper costs. Outsourcing and offshoring represent the two major forms of this job transfer (Spence \& Hlatshwayo, 2011).

Primary among the reasons for acting on the distributional gap between wage earners is the social contract at the heart of America. Spence and Hlatchwayo (2011) reasoned:

Addressing inequality is a complex challenge; almost certainly a multipronged approach will be needed. But the challenge should not be ducked, because the availability of quality employment and the rising gaps in income distribution are politically and socially salient issues, and opportunity is a core piece of the social contract. The absence of rewarding employment opportunities in the lowerand middle-income ranges breaks an important part of the social contract in America, which holds that you are largely on your own but that if you work hard the opportunities will be there (p. 35).

Nontradable, often service related, positions remain domestically, but at stagnant wage increases (Netessine, 2014; Spence \& Hlatchwayo, 2011). Spence and Hlatchwayo (2011) 
offered several potential policy initiatives to help leaders decrease the resultant wage disparity between those in the upper wage category and those in the increasingly growing low wage category. One suggestion was "altering the trends in the tradable sector" (p. 35) by shifting marginal incentives in favor of the U.S. market.

Overall, along with infrastructure and technology improvements, the most important investments into America's ability to afford opportunity for its citizenry are higher education and fundamental research (Spence \& Hlatchwayo, 2011). This conclusion mirrors that of Choi and Mattoni's (2010). To reduce the aspects of vulnerability experienced in the precarious labor force, the authors determined public policy would need to take the form of "pro-employment policies with education reforms and a better enforcement of gender equity measures" (p. 426).

\section{Living Standards}

To ascertain the extent of low wage jobs in South Dakota, this investigation reproduced a study of low wage earners and low wage jobs conducted for the Greater Boston area (Argyres, Holgate, \& Moir, 2012). Argyres et al. utilized data from Occupational Employment Statistics (OES), a database compiled by the BLS, and the ACS, compiled by the U. S. Census Bureau. The OES presents data reported by employers regarding number of employees, hours worked, and wages paid (Argyres et al., 2012). The ACS gathers its "data through annual surveys of individuals who are asked about their current employment" (p. 3). Using data from both employers and employees attempts to mitigate inaccurate reporting, such as identifying one person with two jobs as two workers. The first step for this study is to adopt an income measure of self-sufficiency for households.

The difference between low wages and decent wages has been defined in many ways (Argyres et al., 2012). Argyres et al. defined decent wages as the ability of a full-time worker to meet his or her family's needs without receiving any public subsidies. For a family of four, with two children and two working adults, each adult would need to work full-time at an above minimum wage level to achieve financial independence. Table 1 shows four significant measures of what a threshold wage could be: (a) the Federal Poverty Level, (b) Social Inclusion, (c) Family Economic Self-Sufficiency Standard, and (d) the 1979 Median Wage for Men (Argyres et al., 2012). 
Table 1

Measures of Low Wages and the Corresponding Hourly Wage (2016 dollars) for a Family of Four, requiring 176 hours per month per working adult

\begin{tabular}{|c|c|c|}
\hline Measurement & $\begin{array}{c}\text { Hourly Wage } \\
\text { (2016) }\end{array}$ & Definition \\
\hline $\begin{array}{c}\text { Federal Poverty Level } \\
\$ 24,300 / y e a r\end{array}$ & $\$ 11.68$ & $\begin{array}{l}\text { Computed by the U. S. Department of Health and } \\
\text { Human Services ( } 2016 \text { ), this figure is the result of } \\
\text { dividing the HHS guidelines by } 2080 \text { working } \\
\text { hours in one year. }\end{array}$ \\
\hline $\begin{array}{c}\text { Social Inclusion } \\
\text { Less than two-thirds of } \$ 22.88 / \text { hour } \\
\text { median wage for men }\end{array}$ & $\$ 15.25$ & $\begin{array}{l}\text { Two-thirds of the median wage published by the } \\
\text { BLS (2016a). Examples explained in Boushey et } \\
\text { al. (2007). }\end{array}$ \\
\hline $\begin{array}{c}\text { Family Economic } \\
\text { Self-Sufficiency Standard }\end{array}$ & $\$ 10.86$ & $\begin{array}{l}\text { Adjusted to } 2016 \text { dollars from a University of } \\
\text { Washington study of South Dakota (Pearce \& } \\
\text { Brooks, 2000), derived as a weighted average of } \\
\text { all counties. }\end{array}$ \\
\hline $\begin{array}{c}\text { 1979 Median Wage for Men } \\
\$ 33.59 / \text { hour for a single wage earner as } \\
\text { in } 1979\end{array}$ & $\$ 16.79$ & $\begin{array}{l}\text { Adjusted to } 2016 \text { dollars from a } \\
\text { U. S. Department of Commerce (1979) report } \\
\text { detailing household income, then divided by two } \\
\text { working adults. }\end{array}$ \\
\hline
\end{tabular}

\section{Poverty Guidelines}

The Federal Poverty Level, as defined by the U. S. Department of Health and Human Services (2016), indicated $\$ 24,300$ was needed in 2016 to be at the poverty threshold for a family of four. Therefore, decent wages would be $200 \%$ of that level, or $\$ 48,600$ per year. To attain this level and to avoid dependence on subsidies, a minimum of the poverty level would need to be earned per adult.

\section{Social Inclusion}

Boushey et al. (2007) explained Social Inclusion as a measure that "avoids limitations of the basic income formulas by using a comparison with other jobs to define low-wage work" (p. 4). A job has low wages if it "pays less than two-thirds of the median wage for men" (p. 4); using the wage for men, rather than all workers, removes gender inequity. The BLS published that men averaged $\$ 915$ per week in 2016 , while women averaged $82 \%$ of male wages with $\$ 749$ per week (BLS, 2016a). Therefore, basing the measure on men's wages, the median wage for 2016 would be $\$ 22.88$ per hour (\$915 divided by 40 hours).

Social Inclusion determines that any pay equal to two-thirds $(\$ 15.25)$ or less of the median (\$22.88) would constitute low wages (Boushey et al., 2007). Using the same definition of decent wage as compared to the Federal Poverty Level, if one member of a two-earner 
household had a job at the Social Inclusion level, the burden on the second working adult would be reduced. For example, using the median and Social Inclusion values listed here, the second worker would need to earn only $\$ 8.11$ per hour to reach the subsidy-free level of household income. This type of partnership offers a better chance at an improved, and self-sufficient, standard of living in households with two earners.

\section{Family Economic Self-Sufficiency Standard}

Pearce and Brooks, from the University of Washington's Center for Women's Welfare (CWW), developed the Family Economic Self-Sufficiency Standard (FESS) as a comprehensive valuation of the real costs to sustain different household types without using government subsidies (http://selfsufficiencystandard.org). The researchers have evaluated 39 states since 1996, many several times. South Dakota was evaluated, county by county, once in 2000 (Appendix A).

To validate a statewide FESS value using the most expensive region (MER) was compared to the results of finding a weighted average (WA) for each state. Using the population of each county within each state to assemble a WA yielded a more realistic, statewide composite FESS value. Using Alabama as a test (Table 2), where the only evaluation was done in 2003, the FESS hourly rate for a single earner was weighted by county as a proportion of its contribution to the entire state's population. The same was done after using the Consumer Price Index (CPI) calculator to bring each value to 2011 and 2016 dollars, producing the following results. The MER per hour in 2003 equaled \$9.69, with CPI increases to \$11.74 in 2011 and \$12.63 in 2016. Using the WA method equaled per hour rates of $\$ 8.09$ in 2003, $\$ 9.83$ in 2011, and $\$ 10.62$ in 2016.

Table 2

Alabama Test Case Results

\begin{tabular}{lllllll}
\hline Year & $\begin{array}{l}\text { Most Expensive } \\
\text { Region (MER) } \\
\text { per hour }\end{array}$ & $\begin{array}{l}\text { Weighted } \\
\text { Average (WA) } \\
\text { per hour }\end{array}$ & Change & $\begin{array}{l}\text { Low Wage } \\
\text { Proportion } \\
\text { (MER) }\end{array}$ & $\begin{array}{l}\text { Low Wage } \\
\text { Proportion } \\
\text { (WA) }\end{array}$ & Change \\
\hline 2003 & $\$ 9.69$ & $\$ 8.09$ & $-16.51 \%$ & & & \\
2011 & $\$ 11.74$ & $\$ 9.83$ & $-16.27 \%$ & $54.69 \%$ & $48.24 \%$ & $-11.79 \%$ \\
2016 & $\$ 12.63$ & $\$ 10.62$ & $-15.91 \%$ & $55.57 \%$ & $48.59 \%$ & $-12.56 \%$ \\
\hline $\begin{array}{l}\text { Average change } \\
\text { MER to WA }\end{array}$ & & $-16.23 \%$ & & & $-12.18 \%$ \\
\hline
\end{tabular}

Source: Report published by the Center for Women's Welfare, University of Washington, at http://www.selfsufficiencystandard.org/state-data, and data extracted from Integrated Public Use Microdata Series (IPUMS) USA database for 2006-2010 and 2011-2015, at https://usa.ipums.org.

DOI: 10.17605/OSF.IO/QF4HY 
Alabama's test in Table 2 resulted in an average decline of $16.23 \%$ for self-sufficiency hourly wages from 2011 to 2016. The population under the low wage single earner threshold dropped an average of $12.18 \%$. Workers no longer designated as low wage were 240,169 and 259,007 in 2011 and 2016, respectively. Continuing the WA method for each of the remaining states produced results more salient to national comparison than the MER method, and are discussed at length in the Methodology section.

Compared to other standards options, the FESS attempts to adjust for variations of family size, type, and location by examining every county and major metropolitan area in each state (Pearce \& Brooks, 2000). Researchers from CWW at the University of Washington investigated 39 states to date since 1996, some several times. The dominant areas Pearce and Brooks (2000) found in South Dakota were Rapid City/Pennington County, Sioux Falls/Minnehaha County, Spink County, and Todd County. Keeping with the example of two working adults with two children, the weighted average FESS in 2016 would be $\$ 10.86$ per hour per adult. Two adults working eight hours each day for 22 days each month would generate a monthly income of $\$ 3,823$, or $\$ 45,873$ annually. Table 3 lists different household compositions, in 2016 dollars.

Table 3

Self-Sufficiency Wages in 2016 by Household Structure for South Dakota

\begin{tabular}{lc}
\hline \multicolumn{1}{c}{ Type of Family/Household } & Hourly Wage per Full-Time Working Adult \\
\hline One adult, no children & $\$ 9.06$ \\
$\begin{array}{l}\text { Two adults and two children (one preschool age } \\
\text { and one school age) }\end{array}$ & $\$ 10.86$ \\
$\begin{array}{l}\text { One adult and two children (one preschool age and } \\
\text { one school age) }\end{array}$ & $\$ 20.10$ \\
\hline
\end{tabular}

Note: Reproduced from Pearce and Brooks (2000), The Self-Sufficiency Standard for South Dakota, Table 1 (p. 11); adjusted to 2016 dollars with the CPI calculator on the BLS website (https://data.bls.gov/cgi-bin/cpicalc.pl). Two adult household was calculated as a weighted average of every county within South Dakota.

\section{Median Wage for Men}

Although the current generation of workers enjoys a more technologically advanced world, reaching the standard of living and discretionary income commensurate to a previous generation would call for a comparison to the 1979 Median Wage for Men. From 1979's U. S. Department of Commerce report, "the median income of households maintained by a male householder was $\$ 20,140$, while that of households maintained by a female householder was $\$ 8,520 "$ (p. 3). When adjusted from January 1979 to January 2016 dollars, the median income for men would equal $\$ 69,861$, or $\$ 33.59$ per hour ( $\$ 69,861$ divided by 2080 working hours). In a 
household with two working adults to share the burden of earning this income, each would need average wages of at least $\$ 16.79$ per hour. Argyres et al. (2012) reminded readers that the 1979 median wage also would "need to be accompanied by health insurance and pension benefits to qualify as a good job" (p. 2).

Among potential criticisms of the 1979 Median Wage for Men as a relevant standard is that a person earning nearly $\$ 70,000$ per year in 2016 would put him or her in the top 40th income percentile. Actual national median income for men in 2016 was $\$ 47,580$ (BLS, 2016a), indicating wages have not kept pace with inflation. In fact, median earning in 2016 equaled only $68 \%(47,580 / 69,861)$ of 1979's inflation-adjusted value. This means over the past 37 years, inflation rose $32 \%$ higher than corresponding wages. On the surface, it would appear workers slowly incurred greater costs of living as time passed; however, this conclusion must be balanced by looking at how these workers did and do live.

Responsible reporting must include a consideration as to whether this median wage differential indicates undue stagnation or is a result of radically different needs in 1979 and 2016 populations. As previously mentioned, basic technologies between the two groups have very little in common. The presence of smartphones, cable television, microwave ovens, and energy efficient appliances are but a few of the examples not shared by these groups. Time efficiency and cost of operations are also factors. Due to economies of scale and countless breakthroughs, some jobs no longer exist, and the job descriptions of others have been reinvented. As a result, simply increasing the 1979 median wage by the overall economy's inflation rate over 37 years ignores structural changes in the labor market, transformation of the overall economy, and changes in capital and human stock over multiple generations of workers.

To achieve an accurate and relevant examination of the South Dakota labor market, Integrated Public Use Microdata Series (IPUMS) USA database information could only be useful if a threshold was applied to determine low wage proportions of the work force. Four types of living wages were discussed: (a) the Federal Poverty Level, (b) Social Inclusion, (c) FESS, and (d) the 1979 Median Wage for Men. By utilizing a WA method, FESS prevailed as the most comprehensive and realistic threshold to use when evaluating South Dakota.

\section{METHODOLOGY}

This analysis combined the efforts of two separate university studies in its design. The University of Washington's CWW developed a wage standard used for this analysis's low wage single earner and household level thresholds (Pearce \& Brooks, 2000). Argyres et al.'s (2012) example from the University of Massachusetts Boston enumerated the multiple aspects critical to a thorough evaluation of a labor market (Argyres et al., 2012). Its tables were reproduced, specific to South Dakota. 


\section{University of Washington}

At the University of Washington, Pearce and Brooks (2000) constructed the FESS as a more realistic alternative to the federal minimum wage. The researchers painstakingly determined the appropriate hourly wage each working adult in a South Dakota household needed to earn for that household to be independent of subsidies. Every combination of household, from one adult with no children to two adults with three children of varying ages, was evaluated and assigned an FESS value. Just as convention often uses a family of four when discussing the federal poverty threshold, two adults with one preschool and one school age child was used as this study's benchmark (Pearce \& Brooks, 2000).

Through the CWW, Pearce and Brooks calculated FESS values for 38 states and the District of Columbia. The authors were commissioned to evaluate one or more every year since 1996. CWW's website (http://selfsufficiencystandard.org) provides links to each study, except for the most recent done for Iowa. CWW was contacted to access Iowa's report for this study, but this author received no response. Therefore, Iowa was the only market with an FESS examination not included in this study. Several states have been examined multiple times, others only once, and still a minority of others not at all. The researchers measured each county for households of varying compositions. Even children were identified in different combinations: (a) infant, (b) preschooler, (c) school age, and (d) teenager. Each combination of earners was considered with each possible combination of children (Pearce \& Brooks, 2000).

FESS analyzes the actual cost of living, by county, to determine what income is necessary for each size family to live free of subsidies (Pearce $\&$ Brooks, 2000). The resulting hourly wage consistently exceeded that set by federal minimum wage and federal poverty guidelines for similar households. For this study's national comparison, FESS was used as the threshold for examination in each of these same 38 markets. FESS was examined on a county by county basis, factored with inflation adjustments, but given a WA to establish a state-level minimum hourly wage. A second, compelling reason to use FESS as the threshold for this study was its adoption by University of Massachusetts Boston researchers in their work (Argyres et al., 2012).

\section{University of Massachusetts Boston}

Researchers at the University of Massachusetts Boston explored low wage earners as a proportion of the overall Greater Boston MSA labor force (Argyres et al., 2012). One area of emphasis detected which occupations had a disproportionate number of low wage jobs.

Revealing this information could help identify where income standards, perhaps, needed to be raised to meet the requirements of these workers and their families through the employment workers were already doing. Argyres et al.'s (2012) probe was used as the template for this study's in-depth analysis of South Dakota. 
South Dakota was evaluated on a microdata level to determine what portion of the labor force was not only low wage earners, but also what proportions were:

- full-time (35 or more hrs/wk) versus part-time

- full year (50 or more wks/year)

- public versus private jobs

- $\quad$ self-employed

- unpaid family workers

- military

Data sets were scrutinized in five-year blocks, 2011-2015 and 2006-2010, as a longitudinal effort to identify wage and proportion of labor market trends for each occupation (Ruggles et al., 2015).

OES interquartile wage ranges established a median hourly rate for each of 22 occupational categories (BLS, 2016b). Microdata values for annual income provided low wage concentrations of the labor market, as a statewide percentage and within each occupation (Ruggles et al., 2015). Argyres et al. (2012) charted the types of detailed analysis required to identify areas of development capable of being addressed by public policy

\section{DATA MINING}

To determine South Dakota's composition, U. S. Census and BLS data were analyzed on both the aggregate and microdata levels. IPUMS USA database was accessed on their website (https://usa.ipums.org/usa/), where extracts were compiled for each five-year period. Some of these demographics included such factors as gender, income, location, occupation, and employment variations.

\section{South Dakota Labor Force}

Table 4 lists five-year estimates for "demographic, income, and employment data [in South Dakota] derived from regular surveys of a sample of housing units" (Argyres et al., 2012, p. 3). Overall labor figures were taken from the most recent ACS data set, 2011-2015, and Data Profile Table DP03 (Appendix B). Full-time, part-time, full year, and annual income figures were taken from ACS 2011-2015 Public Use Table S2303, limited to South Dakota, (Appendix C). Both sources were available through the U. S. Census Bureau website. For comparison over time, Table 5 relates the corresponding data for 2006-2010. 
Table 4

Employment Statistics of All and Low Wage Earners in South Dakota (2011-2015)

\begin{tabular}{|c|c|c|c|c|c|}
\hline \multirow[b]{2}{*}{ Characteristics } & \multicolumn{2}{|c|}{ All Workers } & \multicolumn{3}{|c|}{ Low Wage Earners } \\
\hline & $\begin{array}{r}\text { Number } \\
\text { of } \\
\text { workers } \\
\end{array}$ & $\begin{array}{r}\text { Percent of all } \\
\text { workers }\end{array}$ & $\begin{array}{r}\text { Number of low } \\
\text { wage earners }\end{array}$ & $\begin{array}{r}\text { Percent of low } \\
\text { wage earners }\end{array}$ & $\begin{array}{r}\text { Percent of all } \\
\text { workers in } \\
\text { category }\end{array}$ \\
\hline Total workers & 417,540 & $100 \%$ & 178,680 & $100 \%$ & $42.79 \%$ \\
\hline $\begin{array}{l}\text { Employed full-time (35+ } \\
\text { hours/week) }\end{array}$ & 326,516 & $78.20 \%$ & 87,310 & $48.86 \%$ & $26.74 \%$ \\
\hline Employed 1-34 hrs/wk & 95,324 & $22.83 \%$ & 83,752 & $46.87 \%$ & $87.86 \%$ \\
\hline \multicolumn{6}{|c|}{$100 \%$} \\
\hline $\begin{array}{l}\text { Employed full year (50+ } \\
\text { weeks/year) }\end{array}$ & 318,917 & $76.38 \%$ & 104,793 & $58.65 \%$ & $31.38 \%$ \\
\hline $\begin{array}{l}\text { Employed full-time, full } \\
\text { year }\end{array}$ & 276,597 & $66.24 \%$ & 51,413 & $28.77 \%$ & $17.75 \%$ \\
\hline Private sector & 317,372 & $76.01 \%$ & 121,913 & $68.23 \%$ & $38.41 \%$ \\
\hline Public sector & 64,635 & $15.48 \%$ & 36,504 & $20.43 \%$ & $56.48 \%$ \\
\hline Self-employed & 34,405 & $8.24 \%$ & 19,244 & $10.77 \%$ & $55.93 \%$ \\
\hline Unpaid family worker & 1,128 & $0.27 \%$ & 1,019 & $0.57 \%$ & $90.34 \%$ \\
\hline & & $100 \%$ & \multicolumn{3}{|c|}{$100 \%$} \\
\hline
\end{tabular}

Source: ACS Data Profile Table DP03 for 2011-2015 and ACS Public Use Table S2303, limited to South Dakota (U. S. Census, 2016). Low wage earner percentages and numbers generated through microdata examination of IPUMS USA extracts (Ruggles et al., 2015).

Table 5

Employment Statistics of All and Low Wage Earners in South Dakota (2006-2010)

\begin{tabular}{|c|c|c|c|c|c|}
\hline \multirow[b]{2}{*}{ Characteristics } & \multicolumn{2}{|c|}{ All Workers } & \multicolumn{3}{|c|}{ Low Wage Earners } \\
\hline & $\begin{array}{r}\text { Number } \\
\text { of } \\
\text { workers }\end{array}$ & $\begin{array}{r}\text { Percent of all } \\
\text { workers }\end{array}$ & $\begin{array}{l}\text { Number of low } \\
\text { wage earners }\end{array}$ & $\begin{array}{r}\text { Percent of low } \\
\text { wage earners }\end{array}$ & $\begin{array}{r}\text { Percent of all } \\
\text { workers in } \\
\text { category }\end{array}$ \\
\hline Total workers & 392,680 & $100 \%$ & 171,823 & $100 \%$ & $43.76 \%$ \\
\hline $\begin{array}{l}\text { Employed full-time (35+ } \\
\text { hours/week) }\end{array}$ & 262,310 & $66.8 \%$ & 68,489 & $39.86 \%$ & $26.11 \%$ \\
\hline Employed 1-34 hrs/wk & 130,370 & $33.2 \%$ & 101,858 & $59.28 \%$ & $78.13 \%$ \\
\hline \multicolumn{6}{|c|}{$100 \%$} \\
\hline $\begin{array}{l}\text { Employed full year (50+ } \\
\text { weeks/year) }\end{array}$ & 245,818 & $62.6 \%$ & 75,343 & $43.85 \%$ & $30.65 \%$ \\
\hline $\begin{array}{l}\text { Employed full-time, full } \\
\text { year }\end{array}$ & 213,225 & $54.3 \%$ & 36,867 & $21.46 \%$ & $17.29 \%$ \\
\hline Private sector & 291,290 & $74.18 \%$ & 114,657 & $66.73 \%$ & $39.36 \%$ \\
\hline Public sector & 62,711 & $15.97 \%$ & 35,980 & $20.94 \%$ & $57.37 \%$ \\
\hline Self-employed & 36,873 & $9.39 \%$ & 20,258 & $11.79 \%$ & $54.94 \%$ \\
\hline Unpaid family worker & 1,806 & $0.46 \%$ & 928 & $0.54 \%$ & $51.38 \%$ \\
\hline & & $100 \%$ & \multicolumn{3}{|c|}{$100 \%$} \\
\hline
\end{tabular}

Source: ACS Data Profile Table DP03 for 2006-2010 and ACS Public Use Table S2303, limited to South Dakota (U. S. Census, 2016). Low wage earner percentages and numbers generated through microdata examination of IPUMS USA extracts (Ruggles et al., 2015).

DOI: $10.17605 / O S F . I O / Q F 4 H Y$ 
ACS data from Table S2303 (Appendix C) estimated South Dakota's population between 16 to 64 years as 532,191, with 80,997 not working for various reasons. This resulted in a labor force of 451,194, slightly off from the 451,073 values from Table DP03 (Appendix B). After careful examination of the microdata aggregated for these estimates, this author's analysis of the labor force reduced it further by removing military, unemployed, and retired individuals. Consistent with OES occupational categories, South Dakota had 417,540 workers across the 22 designations.

Appendix C, for the 12 months ending May 2016, indicated full-time workers comprised $78.20 \%$ of the work force yielding a total of 326,516 workers. Of those working full-time, $276,597(86.73 \%)$ did so full year. The remaining $13.27 \%$ employed full year ([318,917-276,597]/318,917) maintained only part-time employment. These figures approximate $66.24 \%$ of South Dakota's labor force had full wages throughout the year (Table 4).

Iteration One. To determine the values for Low Wage earners on Table 4 and Table 6, data was extracted from IPUMS for the 5-year ACS period of 2011-2015 (Ruggles et al., 2015). Table 5 and Table 7 reflect similar data extracted for 2006-2010. Within these data sets were variables for census year (YEAR), data set number (DATANUM), household serial number (SERIAL), household weight (HHWT), group quarters status (GQ), person number in sample unit (PERNUM), person weight (PERWT), age (AGE), occupational labels for U. S. Census surveys (OCC2010) and BLS surveys (OCCSOC), and wage and salary income (INCWAGE). Microdata was exported into Excel for analysis.

OES established new codes in 2010 to expand the job titles explicitly assigned within each occupation; this new variable is OCC2010. Line items were sorted by OCC2010, then separated into the 22 categories identified by older OES data (OCCSOC) category codes. Line items with OCC2010 code 9920 (chronically unemployed) and 9950 (never worked) were excluded. To ensure data was separated accurately, OCC2010 and OCCSOC codes were randomly compared. Ten-line items were compared for each category, except when an occupational code did not have 10 job titles, or the job titles of participants were not varied sufficiently. For the IPUMS 2011-2015, 201 occupational titles were verified; for the IPUMS 2006-2010, 208 occupational titles were verified.

Although not a variable within the IPUMS data, frequency was calculated by multiplying person number with person weight. To get an accurate labor force count, line items with zero or blank income values were excluded. Each occupational category was sorted by income in ascending order. Low wage earners were identified as having annual incomes less than the FESS value necessary to take care of a family of four in South Dakota, in 2016 dollars.

Two threshold levels were set - one for each of two working adults in a household, and one with the household's need met by only one income. The microdata sets were for five-year periods, while OES and other ACS data were for snapshots in 2016 and 2011, respectively. Although versions of Table 6 and Table 7 were created with this data, the results could not support the completion of all elements in Table 4 and Table 5. Information regarding public 
Table 6

South Dakota's Low-Wage Earners by Occupation, May 2016

\begin{tabular}{|c|c|c|c|c|c|}
\hline \multirow[b]{2}{*}{ Occupation } & \multicolumn{2}{|c|}{$\begin{array}{c}\text { All Workers } \\
\text { (OES wage data) }\end{array}$} & \multicolumn{3}{|c|}{$\begin{array}{c}\text { Low Wage Earners } \\
\text { (below } \$ 10.86 / \mathrm{hr}, \text { FESS per earner) }\end{array}$} \\
\hline & $\begin{array}{c}\text { Workers in } \\
\text { category }\end{array}$ & $\begin{array}{l}\text { Percent of All } \\
\text { workers }\end{array}$ & $\begin{array}{c}\text { Low wage } \\
\text { workers }\end{array}$ & $\begin{array}{l}\text { Percent of All low } \\
\text { wage workers }\end{array}$ & $\begin{array}{l}\text { Percent of } \\
\text { occupation }\end{array}$ \\
\hline Management & 11,580 & $2.77 \%$ & 2,609 & $1.46 \%$ & $22.53 \%$ \\
\hline $\begin{array}{l}\text { Business \& Financial } \\
\text { Operations }\end{array}$ & 18,630 & $4.46 \%$ & 2,798 & $1.57 \%$ & $15.02 \%$ \\
\hline Computer \& Mathematical & 7,830 & $1.88 \%$ & 1,034 & $0.58 \%$ & $13.20 \%$ \\
\hline $\begin{array}{l}\text { Architecture \& } \\
\text { Engineering }\end{array}$ & 4,840 & $1.16 \%$ & 505 & $0.28 \%$ & $10.43 \%$ \\
\hline $\begin{array}{l}\text { Life, Physical, \& Social } \\
\text { Sciences }\end{array}$ & 4,070 & $0.97 \%$ & 705 & $0.40 \%$ & $17.32 \%$ \\
\hline $\begin{array}{l}\text { Community \& Social } \\
\text { Service }\end{array}$ & 6,550 & $1.57 \%$ & 1,563 & $0.87 \%$ & $23.86 \%$ \\
\hline Legal & 1,660 & $0.40 \%$ & 324 & $0.18 \%$ & $19.49 \%$ \\
\hline $\begin{array}{l}\text { Education, Training \& } \\
\text { Library }\end{array}$ & 24,170 & $5.79 \%$ & 8,921 & $4.99 \%$ & $36.91 \%$ \\
\hline $\begin{array}{l}\text { Arts, Design, } \\
\text { Entertainment, Sports, \& } \\
\text { Media }\end{array}$ & 5,560 & $1.33 \%$ & 2,680 & $1.50 \%$ & $48.21 \%$ \\
\hline $\begin{array}{l}\text { Healthcare Practitioners \& } \\
\text { Technical }\end{array}$ & 29,800 & $7.14 \%$ & 4,664 & $2.61 \%$ & $15.65 \%$ \\
\hline Healthcare Support & 10,540 & $2.52 \%$ & 6,565 & $3.67 \%$ & $62.29 \%$ \\
\hline Protective Service & 6,470 & $1.55 \%$ & 2,278 & $1.28 \%$ & $35.21 \%$ \\
\hline $\begin{array}{l}\text { Food Preparation \& } \\
\text { Serving Related }\end{array}$ & 41,410 & $9.92 \%$ & 37,248 & $20.85 \%$ & $89.95 \%$ \\
\hline $\begin{array}{l}\text { Building \& Grounds } \\
\text { Cleaning \& Maintenance }\end{array}$ & 16,780 & $4.02 \%$ & 11,902 & $6.66 \%$ & $70.93 \%$ \\
\hline Personal Care \& Service & 14,320 & $3.43 \%$ & 9,460 & $5.29 \%$ & $66.06 \%$ \\
\hline Sales \& Related & 47,440 & $11.36 \%$ & 23,269 & $13.02 \%$ & $49.05 \%$ \\
\hline $\begin{array}{l}\text { Office \& Administrative } \\
\text { Support }\end{array}$ & 64,720 & $15.50 \%$ & 26,904 & $15.06 \%$ & $41.57 \%$ \\
\hline $\begin{array}{l}\text { Farming, Fishing, \& } \\
\text { Forestry }\end{array}$ & 1,630 & $0.39 \%$ & 719 & $0.40 \%$ & $44.13 \%$ \\
\hline Construction \& Extraction & 21,940 & $5.25 \%$ & 7,916 & $4.43 \%$ & $36.08 \%$ \\
\hline $\begin{array}{l}\text { Installation, Maintenance, } \\
\text { \& Repair }\end{array}$ & 16,450 & $3.94 \%$ & 3,501 & $1.96 \%$ & $21.28 \%$ \\
\hline Production & 31,600 & $7.57 \%$ & 11,319 & $6.34 \%$ & $35.82 \%$ \\
\hline $\begin{array}{l}\text { Transportation \& Material } \\
\text { Moving }\end{array}$ & 29,550 & $7.08 \%$ & 11,796 & $6.60 \%$ & $39.92 \%$ \\
\hline TOTAL & 417,540 & $100.00 \%$ & 178,680 & $100.00 \%$ & $42.79 \%$ \\
\hline
\end{tabular}

Source: Proportions of each category established by analysis of IPUMS USA (Ruggles et al., 2015) data for 5-year

ACS 2011-2015, then applied to OES snapshots for occupational categories (BLS, 2016b).

(1) Estimates for detailed occupations do not sum to the totals because the totals include occupations not shown separately. Estimates did not include self-employed workers, but low wage proportions did factor in this sector.

(2) Occupations with low wage percentages above $42.79 \%$ indicate low wage workers are over-represented in that industry.

DOI: 10.17605/OSF.IO/QF4HY 
Table 7

South Dakota's Low-Wage Earners by Occupation, May 2011

\begin{tabular}{|c|c|c|c|c|c|}
\hline \multirow[b]{2}{*}{ Occupation } & \multicolumn{2}{|c|}{$\begin{array}{c}\text { All Workers } \\
\text { (OES wage data) }\end{array}$} & \multicolumn{3}{|c|}{$\begin{array}{c}\text { Low-Wage Earners } \\
\text { (below } \$ 9.91 / \mathrm{hr}, \text { FESS per earner) }\end{array}$} \\
\hline & $\begin{array}{c}\text { Workers in } \\
\text { category }\end{array}$ & $\begin{array}{l}\text { Percent of All } \\
\text { workers }\end{array}$ & $\begin{array}{l}\text { Low wage } \\
\text { workers }\end{array}$ & $\begin{array}{l}\text { Percent of All low } \\
\text { wage workers }\end{array}$ & $\begin{array}{l}\text { Percent of } \\
\text { occupation }\end{array}$ \\
\hline Management & 11,820 & $3.01 \%$ & 2,983 & $1.74 \%$ & $25.24 \%$ \\
\hline $\begin{array}{l}\text { Business \& Financial } \\
\text { Operations }\end{array}$ & 16,180 & $4.12 \%$ & 2,527 & $1.47 \%$ & $15.62 \%$ \\
\hline $\begin{array}{l}\text { Computer \& } \\
\text { Mathematical }\end{array}$ & 6,130 & $1.56 \%$ & 1,068 & $0.62 \%$ & $17.42 \%$ \\
\hline $\begin{array}{l}\text { Architecture \& } \\
\text { Engineering }\end{array}$ & 4,360 & $1.11 \%$ & 975 & $0.58 \%$ & $22.37 \%$ \\
\hline $\begin{array}{l}\text { Life, Physical, \& Social } \\
\text { Sciences }\end{array}$ & 3,780 & $0.96 \%$ & 1,161 & $0.68 \%$ & $30.71 \%$ \\
\hline $\begin{array}{l}\text { Community \& Social } \\
\text { Services }\end{array}$ & 6,030 & $1.54 \%$ & 1,157 & $0.67 \%$ & $19.19 \%$ \\
\hline Legal & 1,650 & $0.42 \%$ & 240 & $0.14 \%$ & $14.52 \%$ \\
\hline $\begin{array}{l}\text { Education, Training \& } \\
\text { Library }\end{array}$ & 23,430 & $5.97 \%$ & 7,676 & $4.47 \%$ & $32.76 \%$ \\
\hline $\begin{array}{l}\text { Arts, Design, } \\
\text { Entertainment, Sports, } \\
\text { \& Media }\end{array}$ & 5,530 & $1.41 \%$ & 2,891 & $1.68 \%$ & $52.28 \%$ \\
\hline $\begin{array}{l}\text { Healthcare Practitioners } \\
\& \text { Technical }\end{array}$ & 26,020 & $6.63 \%$ & 4,049 & $2.36 \%$ & $15.56 \%$ \\
\hline Healthcare Support & 10,840 & $2.76 \%$ & 6,740 & $3.92 \%$ & $62.18 \%$ \\
\hline Protective Service & 6,120 & $1.56 \%$ & 2,197 & $1.28 \%$ & $35.90 \%$ \\
\hline $\begin{array}{l}\text { Food Preparation \& } \\
\text { Serving Related }\end{array}$ & 38,590 & $9.83 \%$ & 33,851 & $19.70 \%$ & $87.72 \%$ \\
\hline $\begin{array}{l}\text { Building \& Grounds } \\
\text { Cleaning \& } \\
\text { Maintenance }\end{array}$ & 15,790 & $4.02 \%$ & 11,392 & $6.63 \%$ & $72.15 \%$ \\
\hline Personal Care \& Service & 12,320 & $3.14 \%$ & 8,785 & $5.11 \%$ & $71.31 \%$ \\
\hline Sales \& Related & 45,100 & $11.49 \%$ & 22,442 & $13.06 \%$ & $49.76 \%$ \\
\hline $\begin{array}{l}\text { Office \& Administrative } \\
\text { Support }\end{array}$ & 66,150 & $16.85 \%$ & 27,512 & $16.01 \%$ & $41.59 \%$ \\
\hline $\begin{array}{l}\text { Farming, Fishing, \& } \\
\text { Forestry }\end{array}$ & 1,380 & $0.35 \%$ & 902 & $0.52 \%$ & $65.36 \%$ \\
\hline $\begin{array}{l}\text { Construction \& } \\
\text { Extraction }\end{array}$ & 20,290 & $5.17 \%$ & 7,272 & $4.23 \%$ & $35.84 \%$ \\
\hline $\begin{array}{l}\text { Installation, } \\
\text { Maintenance, \& Repair }\end{array}$ & 14,520 & $3.70 \%$ & 4,121 & $2.40 \%$ & $28.38 \%$ \\
\hline Production & 27,920 & $7.11 \%$ & 9,350 & $5.44 \%$ & $33.49 \%$ \\
\hline $\begin{array}{l}\text { Transportation \& } \\
\text { Material Moving }\end{array}$ & 28,730 & $7.32 \%$ & 12,532 & $7.29 \%$ & $43.62 \%$ \\
\hline TOTAL & 392,680 & $100.0 \%$ & 171,823 & $100.00 \%$ & $43.76 \%$ \\
\hline
\end{tabular}

Source: Proportions of each category established by analysis of IPUMS USA (Ruggles et al., 2015) data for 5-year ACS 2006-2010, then applied to OES snapshots for occupational categories (BLS, 2016c).

(1) Estimates for detailed occupations do not sum to the totals because the totals include occupations not shown separately. Estimates did not include self-employed workers, but low wage proportions did factor in this sector.

(2) Occupations with low wage percentages above $43.76 \%$ indicate low wage workers are over-represented in that industry. 
versus private occupations, as well as information for self-employed persons and those designated as unpaid family workers within each occupation were not evident. To better understand how these factors for Table 4 and Table 5 were determined, this researcher contacted the authors of the Greater Boston labor force study (Argyres et al., 2012). Dr. Susan Moir and Dr. Brandynn Holgate were able to respond quickly to the inquiry.

Iteration Two. Dr. Holgate revealed their study's data (Argyres et al., 2012) involved extracting more variables than this research had obtained. Values for employment status (EMPSTAT), class of worker (CLASSWKR), weeks worked (WKSWORK2), and usual hours worked (UHRSWORK) offered the necessary information to determine full-time, full year, public, private, self-employed, and unpaid family worker sectors for Table 4. Further, Dr. Holgate argued removing self-employed individuals from Iteration One excluded "an important pool of low-wage workers" (personal communications, July 24, 2017). In deference to her expertise, new data was extracted from IPUMS for each of the five-year periods. The additional variable of earned income (INCEARN) replaced income from wages (INCWAGE), as INCEARN included both wages and other forms of income generation.

One surprisingly pleasant effect of having the additional variables was the ability for this researcher to remove all previous subjectivity used to include and exclude line items.

Employment status (EMPSTAT) had four designations: (a) 0, never worked; (b) 1, employed; (c) 2, unemployed; and (d) 3, not in labor force. Given the ages of a number of those listed as EMPSTAT $=3$, many in this group were retired. EMPSTAT $=0$ were exclusively minors under the age of sixteen. By excluding all line items with 0 or 3 , the only data remaining were employed and unemployed. Calculations for frequency yielded population counts for these two groups.

Separation of unemployed persons allowed for a total count, used as a comparison to snapshot data to verify proportions within the labor force. Having only EMPSTAT = 1 values remaining for occupational counts and income evaluation enabled this researcher to proceed with greater confidence in the resultant values. Class of worker (CLASSWKR) identified public (government and nonprofit) sector, private sector, unpaid family workers, and military. Each of these sectors were separated and counted before reintegration for occupational category evaluation. Military line items remained excluded; OCC2010 codes military with a 9800 series, which is not included in the overall 22 categories.

Microdata established the appropriate proportion of low wage earners within each occupational category. The proportion was applied to the snapshot values listed in Table 6 and Table 7, for example, to determine the number of individual participants in each category considered low wage earners (Appendix D). Appendix E summarizes the author's analysis of wage earners in South Dakota, with the previously described line item exceptions, to establish proportions applied to OES data in Tables 6 and 7. 
Pay Scales. May 2016 interquartile wages for multiple occupations in one geographical area, the state of South Dakota, were accessed through the OES database (BLS, 2016b; Appendix D). Upper and lower values of each wage range were calculated to establish boundaries for the occupational categories, as illustrated in Figure 4. The dark horizontal line represents an approximation of South Dakota's FESS threshold of \$10.86 per hour. Using the Management category as an example, the 10th percentile hourly wage for this occupation was $\$ 22.22$; therefore, the lowest wage in the category would be $\$ 20.20$ (\$22.22/1.1). Similarly, the 90th percentile hourly wage was $\$ 85.00$, resulting in the maximum wage of $\$ 94.44(\$ 85.00 / 0.9)$. Each occupation has a vertical line on Figure 4, spanning the range of wages reported in that category. A triangle on each vertical line marks the occupation's median wage; for Management, the median wage is $\$ 44.14$ per hour. In all categories, Figure 4 illustrates the median wage as significantly below the mean wage for each occupation.

May 2011 interquartile wages also were accessed from the OES database (BLS, 2016c; Appendix D). Values were standardized to 2011 dollars to yield an FESS value of \$9.91 per hour for each of two wage earners in a household. The combined annual income of these wages would need to equal $\$ 41,860$; this value assumes equal contribution of $\$ 20,930$ per year (\$9.91/hour x 8 hours/day x 22 days/month x 12 months) by each adult. Realistically, either or both wage earners may not reach full-time, full year status - consistently working 35 or more hours each week for the entire year.

South Dakota's Department of Labor and Regulation (2016) announced a cost of living increase of 1.06 percent for 2016, resulting in the state minimum wage increasing to $\$ 8.65$ per hour beginning January 1, 2017. Only occupations with wage ranges near the minimum wage, however, directly are affected by the increase. There is no mandate for all wages to increase by 1.06 percent. Figure 4 shows only the Food Service category had a median wage below the 2016 FESS threshold of \$10.86 per hour; Figure 5 indicates the same for the 2011 FESS threshold of $\$ 9.91$ per hour. Given often minimal requisites for employment in this occupation, it is not surprising the Food Service category had the third largest number $(41,410)$ of workers; only Office and Administrative Support $(64,720)$ and Sales $(47,440)$ had more workers.

Food Preparation and Serving had the smallest wage range, indicating little opportunity to make substantial increases in hourly wages through advancements. This category's wages ranged from $\$ 8.07$ to $\$ 15.09$ per hour, in 2016 (Figure 4). Due to the nature of servers receiving tips, Food Service is one occupation where employers legally can pay less than the state minimum wage. South Dakota's Department of Labor and Regulation (2016) explained the hourly minimum wage for tipped employees is half the wage for non-tipped employees. "Wages and tips combined must equal at least the minimum wage" (para. 3). This structure assumes all servers will earn at least half the minimum wage in tips each hour. 


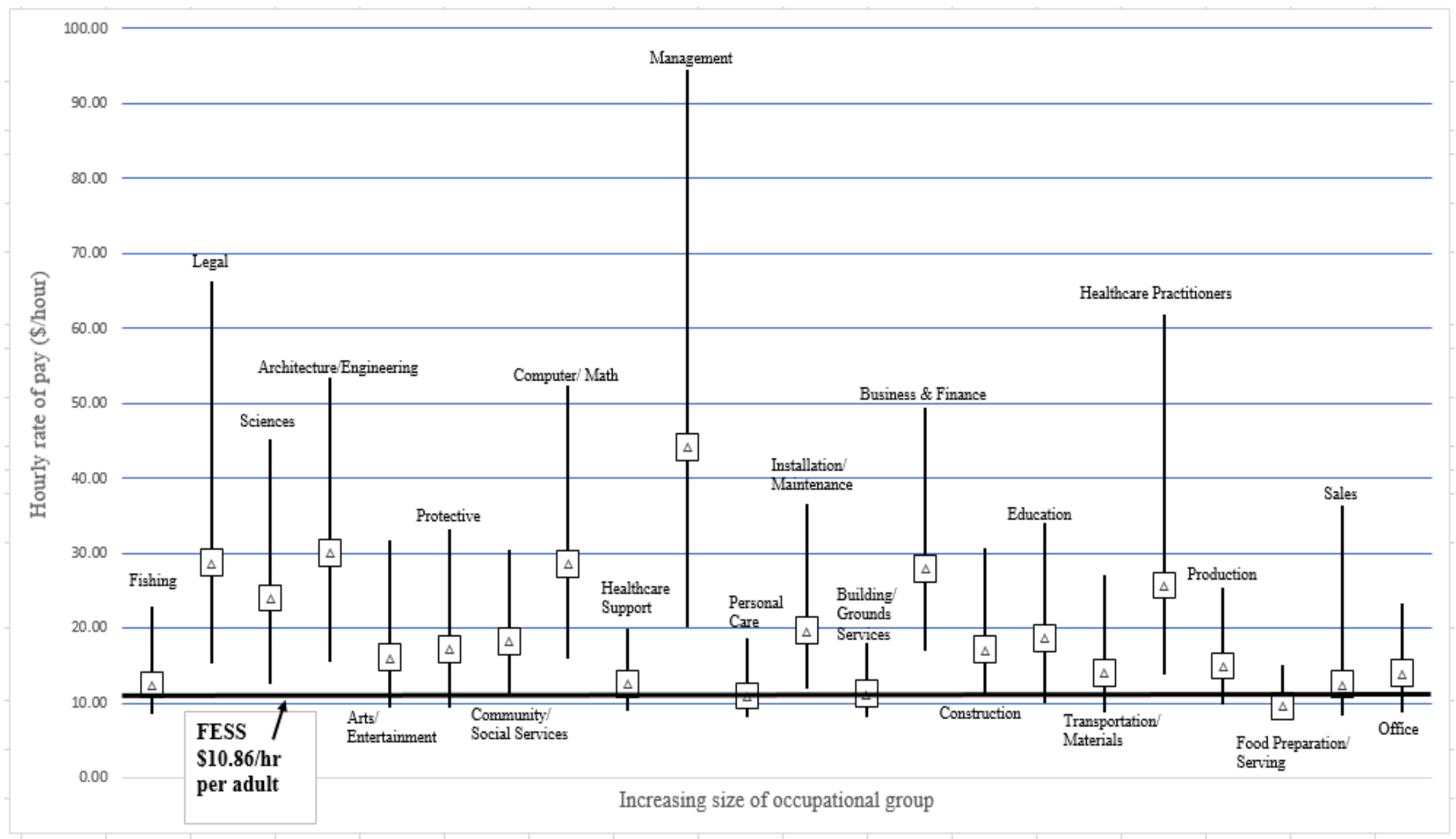

Figure 4. Interquartile hourly wage ranges by major occupations f South Dakota, 2016. Reference: Bureau of Labor Statistics. (2016b). Occupational Employment Statistics (OES) data tables. Multiple occupations for one geographical area, South Dakota, May 2016. Retrieved from https://data.bls.gov/oes/ 


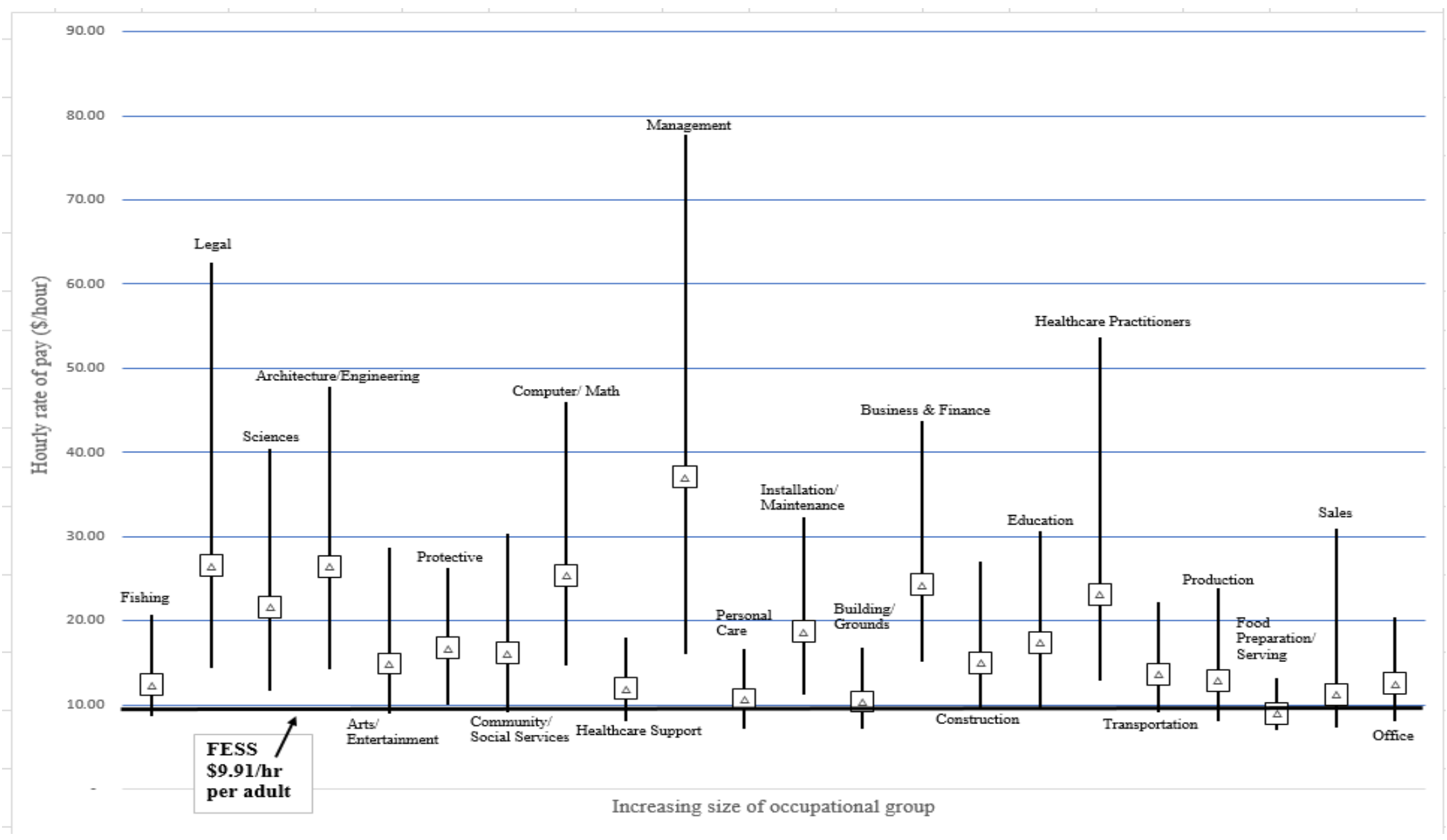

Figure 5. Interquartile hourly wage ranges by major occupations for South Dakota, 2011. Source information obtained from Bureau of Labor Statistics. (2016c). Occupational Employment Statistics (OES) data tables. Multiple occupations for one geographical area, South Dakota, May 2011. Retrieved from https://data.bls.gov/oes/ 


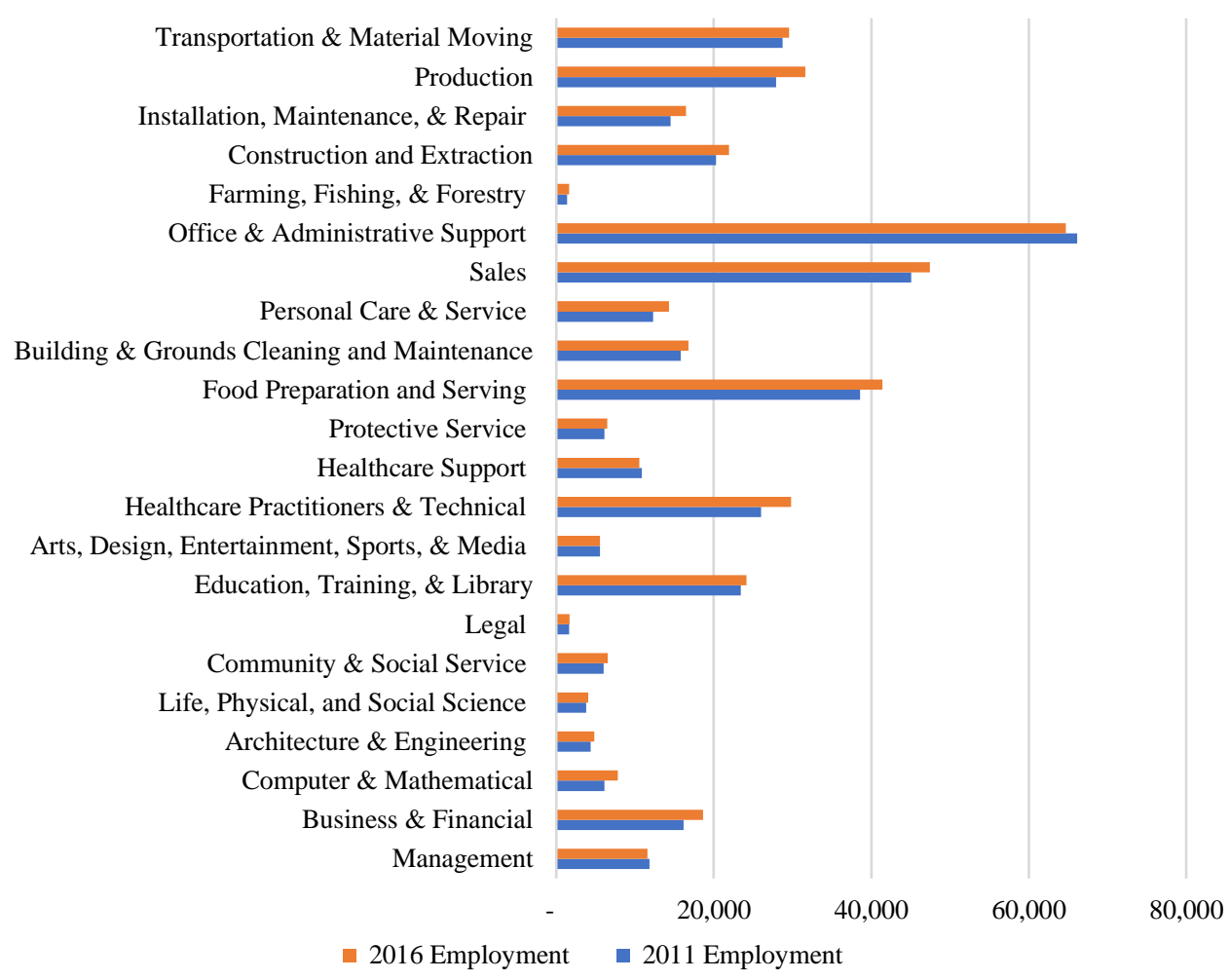

Figure 6. Population by occupation 2011 through 2016. Information represents author's analysis of OES survey data for 2011 and 2016 (BLS, 2016b; BLS, 2016c).

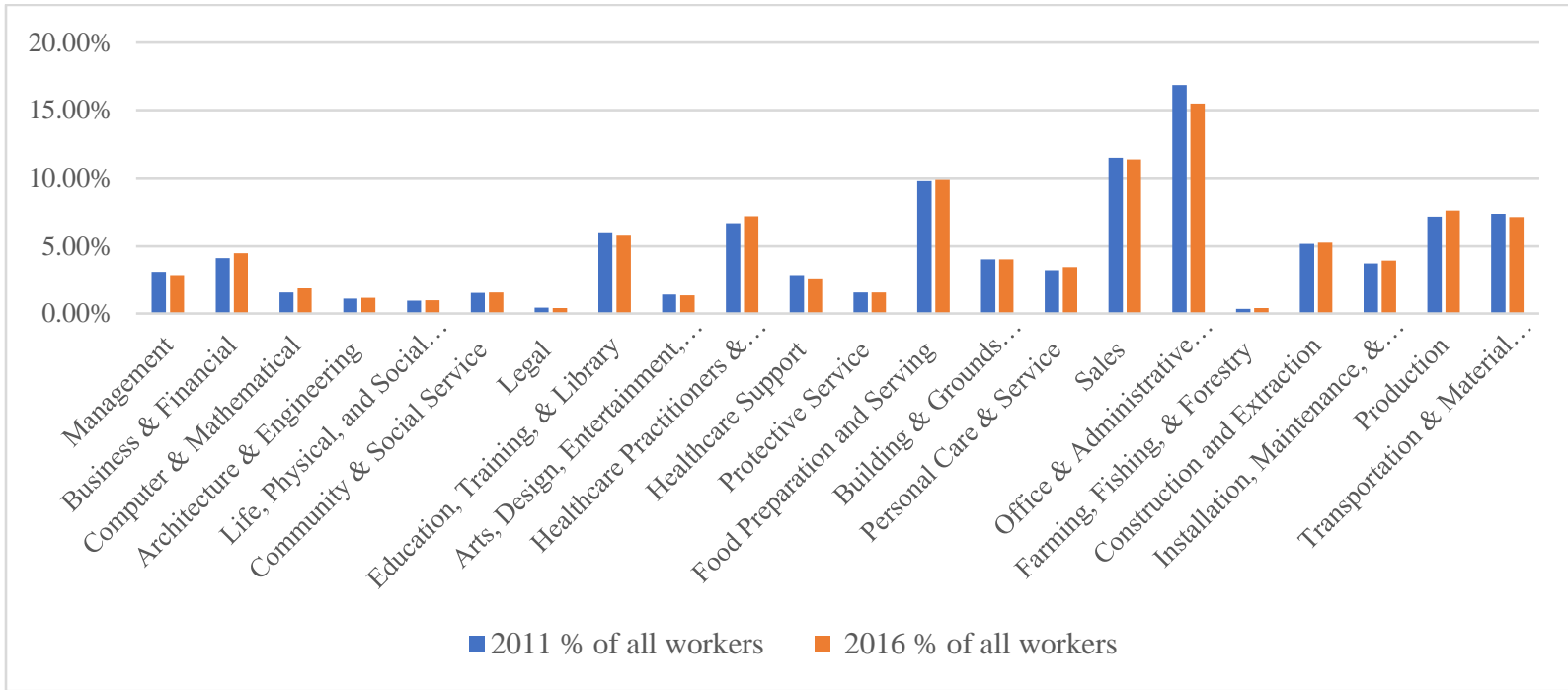

Figure 7. South Dakota labor force in all occupations from 2011 to 2016. Information represents author's analysis of OES survey data for 2011 and 2016 (BLS, 2016b; BLS, 2016c).

DOI: $10.17605 / \mathrm{OSF} . \mathrm{IO} / \mathrm{QF} 4 \mathrm{HY}$ 


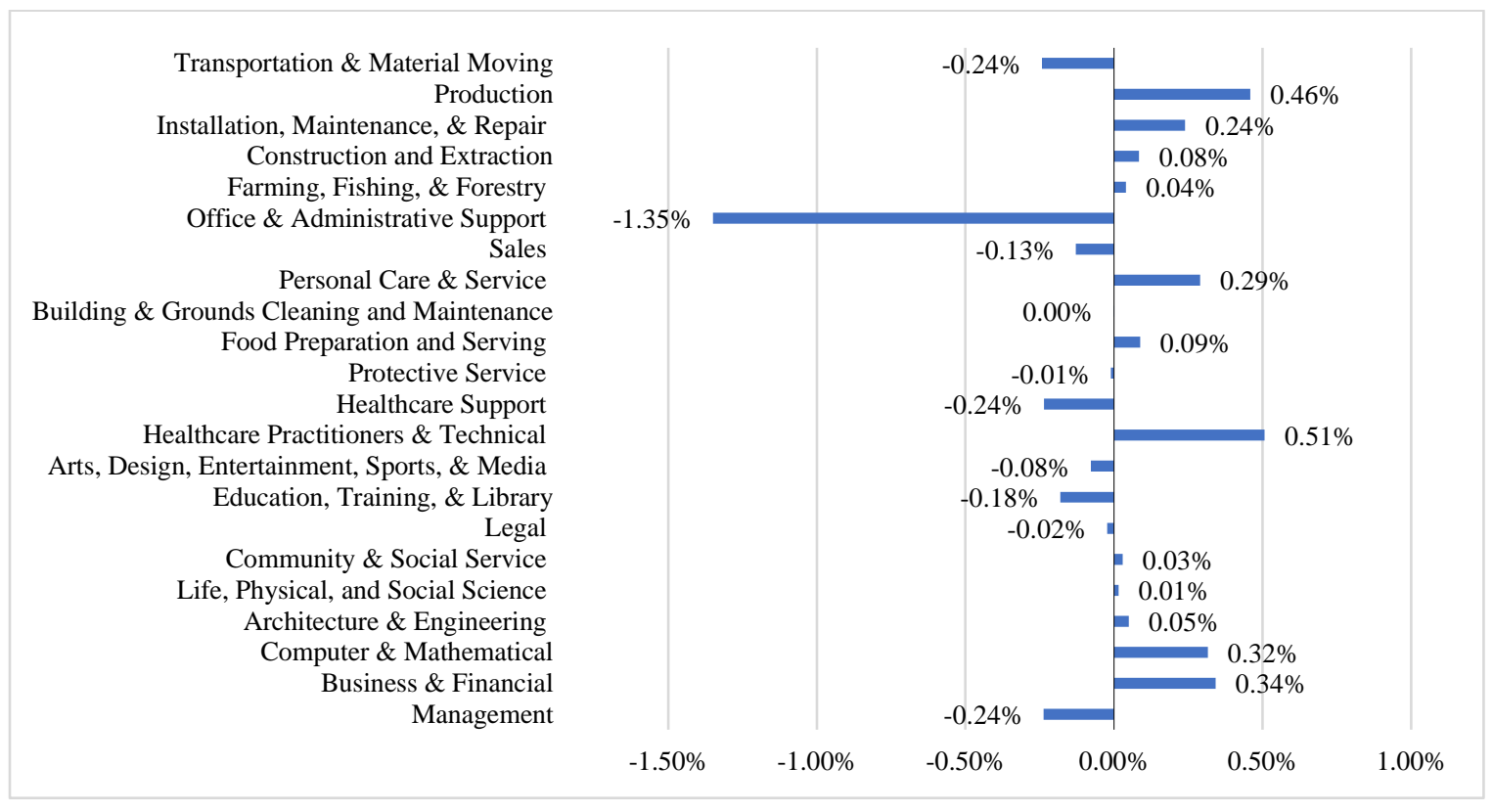

Figure 8. Percentage change in labor force by occupation between 2011 to 2016. Information represents author's analysis of OES survey data for 2011 and 2016 (BLS, 2016b; BLS, 2016c).

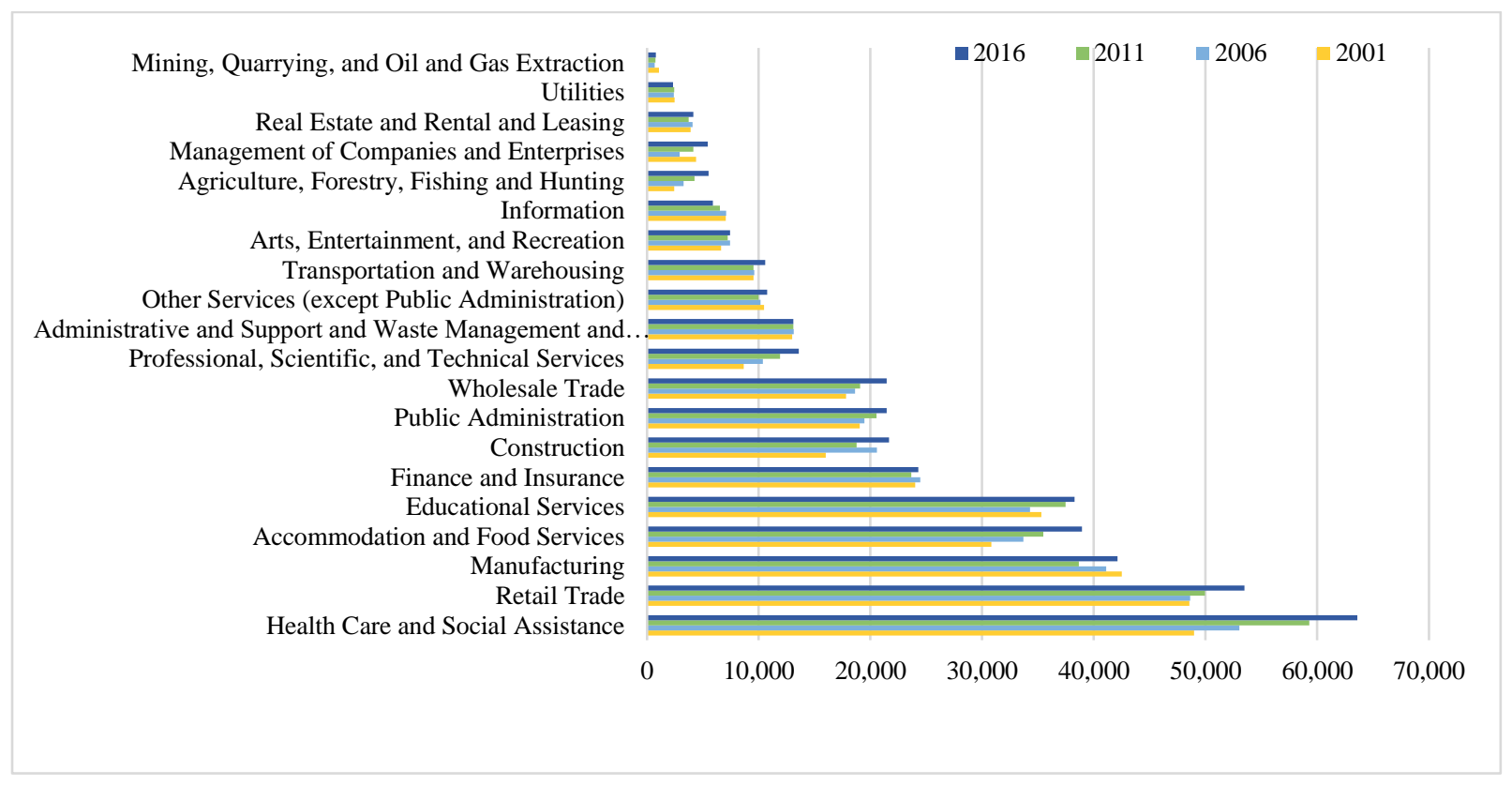

Figure 9. Long-term changes in sector populations, South Dakota 2001-2016. Adapted from U. S. Census Bureau's Longitudinal Employer-Household Dynamics (LEHD, 2017). Quarterly workforce indicators (QWI) data extraction, limited to quarter 2 in years 2001, 2006, 2011, and 2016. 
By comparison, the occupation with the largest pay range was Management; wages for managers in South Dakota were reported as \$20.20 to \$94.44 per hour (Figure 4 ). For a typical 2,080-hour work year, a restaurant host may earn $\$ 16,786$ per year $(\$ 8.07 / \mathrm{hr})$ while some business executives report earning $\$ 196,435$ per year $(\$ 94.44 / \mathrm{hr})$ - before bonuses and options. With occupational ranges much wider in the professional fields than in support staff positions, and the observation that occupational median wages fall below the mean of each range, delving into the raw data for South Dakota's labor force became necessary to get a clearer picture. Figures 6 through 9 show changes in each occupation over time.

Typologies. When examining an occupational category, distribution of jobs within that occupation would indicate an ability for workers to advance from entry level to higher paying ranks. Interquartile median wages were examined to organize each of the 22 occupational categories into one of three tiers - Low Wage, Second Tier, and Higher Wage. These typologies adhere to the example Argyres et al. (2012) set with their investigation of Boston's labor force.

An occupation was selected as Low Wage when its median wage fell below the FESS threshold of $\$ 10.86$ per hour for 2016 , and $\$ 9.91$ per hour for 2011. A version of Social Inclusion theory (Boushey et al., 2007) qualified an occupation as Second Tier if two-thirds of its median wage fell below the FESS threshold. The remaining occupational categories composed the Higher Wage typology, where two-thirds the median wage was above the FESS threshold. Higher Wage occupations do not necessarily indicate incomes equal to upper middle-class wealth, but do suggest wage earners may be able to pay for more than their equitable half of the family's needs.

Of considerable note is that a large proportion of those workers in Second Tier occupations, with better hourly wages, may still be part of the low wage earners listed on OES and ACS data. Although the hourly wages may exceed the self-sufficiency threshold, workers may not have the ability, or desire, to work 35 or more hours each week. Therefore, Table 6 and Table 7 must be viewed in conjunction with Table 8 and Table 9. All 22 occupational groups fit into three possible typologies. Table 8 and Table 9 describe these typologies for 2016 and 2011, respectively.

Second Tier occupations encompass many service-oriented positions, often characterized by less than full-time hours. These categories represent low enough median wages that two-thirds the occupation's median wage does not meet the FESS threshold. These at-risk, and questionably lucrative occupations, with their median wages in 2016 are:

- $\quad$ Personal Care $(\$ 10.97 /$ hour $)$

- $\quad$ Building and Grounds Services (\$11.25/hour)

- $\quad$ Farming, Fishing, and Forestry $(\$ 12.52 /$ hour $)$

- Transportation and Material Moving $(\$ 14.06 /$ hour $)$

- $\quad$ Production ( $\$ 14.98 /$ hour)

- $\quad$ Arts, Entertainment, Sports, and Media ( $\$ 16.15 /$ hour)

DOI: 10.17605/OSF.IO/QF4HY 
Table 8

South Dakota Employment and Wages by Occupational Typology, 2016

$\begin{array}{cccccc}\begin{array}{c}\text { Occupational } \\ \text { Typology }\end{array} & \text { Occupational Title } & \begin{array}{c}\text { Number } \\ \text { Employed }\end{array} & \begin{array}{c}\text { Percent of } \\ \text { Total } \\ \text { Employment }\end{array} & \begin{array}{c}\text { Median } \\ \text { Hourly } \\ \text { Wage }\end{array} & \begin{array}{c}\text { Median } \\ \text { Median }\end{array}\end{array}$

FESS threshold value for $2016=\$ 10.86 /$ hour

\begin{tabular}{|c|c|c|c|c|c|}
\hline \multirow{2}{*}{ 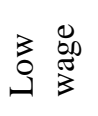 } & Food Preparation/Serving & 41,410 & $9.92 \%$ & \multirow[t]{2}{*}{$\$ 9.62$} & \\
\hline & Low wage total & 41,410 & $9.92 \%$ & & \\
\hline \multirow{11}{*}{ 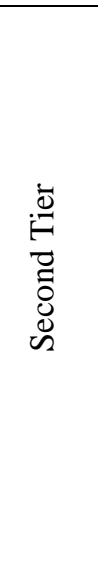 } & Personal Care & 14,320 & $3.43 \%$ & $\$ 10.97$ & $\$ 7.31$ \\
\hline & Building/Grounds Services & 16,780 & $4.02 \%$ & $\$ 11.25$ & $\$ 7.50$ \\
\hline & Sales & 47,440 & $11.36 \%$ & $\$ 12.49$ & $\$ 8.33$ \\
\hline & Farming, Fishing, \& & 1,630 & $0.39 \%$ & $\$ 12.52$ & $\$ 8.35$ \\
\hline & Forestry & & & & \\
\hline & Healthcare Support & 10,540 & $2.52 \%$ & $\$ 12.63$ & $\$ 8.42$ \\
\hline & $\begin{array}{l}\text { Office \& Administrative } \\
\text { Support }\end{array}$ & 64,720 & $15.50 \%$ & $\$ 14.00$ & $\$ 9.33$ \\
\hline & $\begin{array}{l}\text { Transportation \& Material } \\
\text { Moving }\end{array}$ & 29,550 & $7.08 \%$ & $\$ 14.06$ & $\$ 9.37$ \\
\hline & Production & 31,600 & $7.57 \%$ & $\$ 14.98$ & $\$ 9.99$ \\
\hline & Arts/Entertainment & 5,560 & $1.33 \%$ & $\$ 16.15$ & $\$ 10.77$ \\
\hline & Second Tier total & 222,140 & $53.20 \%$ & & \\
\hline \multirow{14}{*}{ 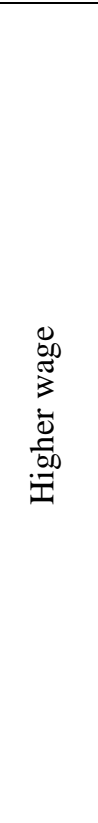 } & Construction \& Extraction & 21,940 & $5.25 \%$ & $\$ 17.13$ & $\$ 11.42$ \\
\hline & Protective Service & 6,470 & $1.55 \%$ & $\$ 17.21$ & $\$ 11.47$ \\
\hline & $\begin{array}{l}\text { Community \& Social } \\
\text { Service }\end{array}$ & 6,550 & $1.57 \%$ & $\$ 18.26$ & $\$ 12.17$ \\
\hline & $\begin{array}{l}\text { Education, Training, \& } \\
\text { Library }\end{array}$ & 24,170 & $5.79 \%$ & $\$ 18.66$ & $\$ 12.44$ \\
\hline & $\begin{array}{l}\text { Installation, Maintenance } \\
\text { \& Repair }\end{array}$ & 16,450 & $3.94 \%$ & $\$ 19.67$ & $\$ 13.11$ \\
\hline & $\begin{array}{l}\text { Life, Physical, \& Social } \\
\text { Sciences }\end{array}$ & 4,070 & $0.97 \%$ & $\$ 24.03$ & $\$ 16.02$ \\
\hline & Healthcare Practitioners & 29,800 & $7.14 \%$ & $\$ 25.70$ & $\$ 17.13$ \\
\hline & Business \& Financial & 18,630 & $4.46 \%$ & $\$ 27.93$ & $\$ 18.62$ \\
\hline & Computer \& Mathematical & 7,830 & $1.88 \%$ & $\$ 28.63$ & $\$ 19.09$ \\
\hline & Legal & 1,660 & $0.40 \%$ & $\$ 28.76$ & $\$ 19.09$ \\
\hline & $\begin{array}{l}\text { Architecture \& } \\
\text { Engineering }\end{array}$ & 4,840 & $1.16 \%$ & $\$ 30.09$ & $\$ 20.06$ \\
\hline & Management & 11,580 & $2.77 \%$ & $\$ 44.14$ & $\$ 29.43$ \\
\hline & Higher wage total & 153,990 & $36.88 \%$ & & \\
\hline & All Jobs & 417,540 & $100.00 \%$ & & \\
\hline
\end{tabular}

Source: Bureau of Labor Statistics. (2016b). Occupational Employment Statistics (OES) data tables. Multiple occupations for one geographical area, South Dakota, May 2016. Retrieved from https://data.bls.gov/oes/ 
Table 9

South Dakota Employment and Wages by Occupational Typology, 2011

$\begin{array}{cccccc}\begin{array}{c}\text { Occupational } \\ \text { Typology }\end{array} & \text { Occupational Title } & \begin{array}{c}\text { Number } \\ \text { Employed }\end{array} & \begin{array}{c}\text { Percent of } \\ \text { Total } \\ \text { Employment }\end{array} & \begin{array}{c}\text { Median } \\ \text { Hourly } \\ \text { Wage }\end{array} & \begin{array}{c}2 / 3 \\ \text { Median }\end{array}\end{array}$

FESS threshold value for $2011=\$ 9.91 /$ hour

\begin{tabular}{|c|c|c|c|c|c|}
\hline \multirow{2}{*}{ 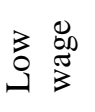 } & Food Preparation/Serving & 38,590 & $9.83 \%$ & \multirow[t]{2}{*}{$\$ 8.95$} & \\
\hline & Low wage total & $\mathbf{3 8 , 5 9 0}$ & $9.83 \%$ & & \\
\hline \multirow{9}{*}{ 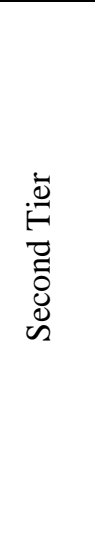 } & Personal Care & 12,320 & $3.14 \%$ & $\$ 10.22$ & $\$ 6.81$ \\
\hline & Building/Grounds & 15,790 & $4.02 \%$ & $\$ 10.40$ & $\$ 6.93$ \\
\hline & $\begin{array}{l}\text { Services } \\
\text { Sales }\end{array}$ & 45,100 & $11.49 \%$ & $\$ 11.23$ & $\$ 7.49$ \\
\hline & Healthcare Support & 10,840 & $2.76 \%$ & $\$ 11.46$ & $\$ 7.64$ \\
\hline & $\begin{array}{l}\text { Farming, Fishing, \& } \\
\text { Forestry }\end{array}$ & 1,380 & $0.35 \%$ & $\$ 12.43$ & $\$ 8.29$ \\
\hline & $\begin{array}{l}\text { Office \& Administrative } \\
\text { Support }\end{array}$ & 66,150 & $16.85 \%$ & $\$ 12.52$ & $\$ 8.35$ \\
\hline & $\begin{array}{l}\text { Transportation \& Material } \\
\text { Moving }\end{array}$ & 28,730 & $7.32 \%$ & $\$ 12.89$ & $\$ 8.59$ \\
\hline & Production & 27,920 & $7.11 \%$ & $\$ 13.68$ & $\$ 9.12$ \\
\hline & Second Tier total & 208,230 & $53.04 \%$ & & \\
\hline \multirow{15}{*}{ 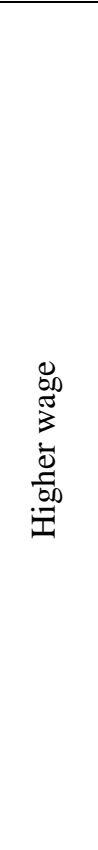 } & Arts/Entertainment & 5,530 & $1.41 \%$ & $\$ 14.88$ & $\$ 9.92$ \\
\hline & Construction \& Extraction & 20,290 & $5.17 \%$ & $\$ 14.98$ & $\$ 9.99$ \\
\hline & Protective Service & 6,120 & $1.56 \%$ & $\$ 16.13$ & $\$ 10.75$ \\
\hline & $\begin{array}{l}\text { Community \& Social } \\
\text { Service }\end{array}$ & 6,030 & $1.54 \%$ & $\$ 16.73$ & $\$ 11.15$ \\
\hline & $\begin{array}{l}\text { Education, Training, \& } \\
\text { Library }\end{array}$ & 23,430 & $5.97 \%$ & $\$ 17.44$ & $\$ 11.63$ \\
\hline & $\begin{array}{l}\text { Installation, Maintenance } \\
\text { \& Repair }\end{array}$ & 14,520 & $3.70 \%$ & $\$ 18.70$ & $\$ 12.47$ \\
\hline & $\begin{array}{l}\text { Life, Physical, \& Social } \\
\text { Sciences }\end{array}$ & 3,780 & $0.96 \%$ & $\$ 21.61$ & $\$ 14.41$ \\
\hline & Healthcare Practitioners & 26,020 & $6.63 \%$ & $\$ 23.10$ & $\$ 15.40$ \\
\hline & Business \& Financial & 16,180 & $4.12 \%$ & $\$ 24.28$ & $\$ 16.19$ \\
\hline & Computer \& Mathematical & 6,130 & $1.56 \%$ & $\$ 25.37$ & $\$ 16.91$ \\
\hline & $\begin{array}{l}\text { Architecture \& } \\
\text { Engineering }\end{array}$ & 4,360 & $1.11 \%$ & $\$ 26.45$ & $\$ 17.63$ \\
\hline & Legal & 1,650 & $0.42 \%$ & $\$ 26.52$ & $\$ 17.68$ \\
\hline & Management & 11,820 & $3.01 \%$ & $\$ 37.08$ & $\$ 24.72$ \\
\hline & Higher wage total & 145,860 & $37.16 \%$ & & \\
\hline & All Jobs & 392,680 & $100.03 \%$ & & \\
\hline
\end{tabular}

Source: Bureau of Labor Statistics. (2016c). Occupational Employment Statistics (OES) data tables. Multiple occupations for one geographical area, South Dakota, May 2011. Retrieved from https://data.bls.gov/oes/ 
Comparing Table 8 and Table 9 reveals that over the 2006-2015 period, only Arts, Entertainment, Sports, and Media changed its tier status. Arts and Entertainment dropped from the lowest category in the Higher Wage tier in 2011, to the highest paid category in the Second Tier in 2016. When less than one dollar per hour can change an occupational category from a somewhat secure income (Higher Wage) to a questionably adequate income (Second Tier), further investigation becomes necessary to determine how much control workers have regarding their hours.

Do most members of an occupation work in the lower range, with a handful of highly paid individuals at the top pulling the median wage higher? When examining individual earnings, will large portions of Second Tier job holders earn low incomes annually? Are employees working the hours they want to make the income they need? How remarkable is South Dakota's labor market when compared to other states?

Workers by Age. Determining the age levels of workers could aid policymakers by highlighting populations in need of economic development initiatives, such as forewarn of an aging population with few retirement alternatives. Building on the examination of occupational typologies, and with consideration to information expressed as "interesting to know" by South Dakota State Senator Reynold Nesiba during an informal interview (April 20, 2017), the IPUMS data previously separated into occupations was dissected further into age groups. Five major groups, with the ideal perceptions of each stage, follow:

(a) 16-29 years of age - presumably at the beginning of a career

(b) 30-39 years of age - building credibility and status within a career

(c) 40-49 years of age - secure in a career and approaching its pinnacle

(d) 50-64 years of age - mature in a career and approaching retirement

(e) 65 years of age and above - working as a distraction in retirement

This author acknowledges the changing landscape that does not confine individuals to career dimensions based solely on age, as evidenced by the increased numbers of individuals pursuing advanced educations later in life. Grouping South Dakota's work force into these five categories, along with investigating the income levels of each group may still offer relevant information. Appendix F details IPUMS data, converted to OES parameters, for each age group within each occupation by worker count and percentage of total work force.

Figure 10 presents age groups as proportions of the entire labor market. Table 10 breaks down each age group by income typology. Figure 11 shows the changes in all and low wage earners, by age groups, for each five-year block of data. Table 11 restates counts of age groups, income levels, and change in each over the ten years analyzed. Although 2016 OES data (BLS, 2016a) indicated an increase of 24,860 workers over 2011 figures, age groups did not progress equally.

DOI: 10.17605/OSF.IO/QF4HY 


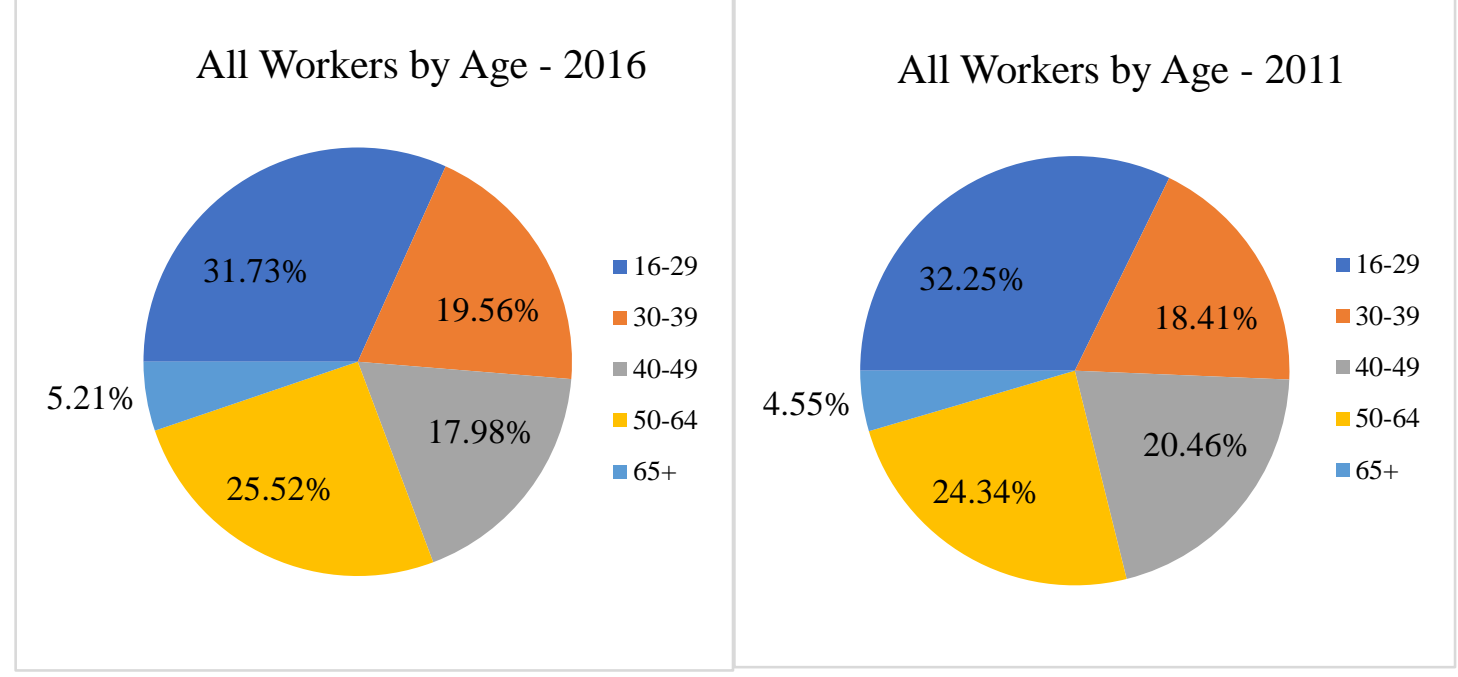

Figure 10. Percentage of all workers by age groups, 2011 and 2016. Source: IPUMS ACS 5-yr data for periods 2006-2010 and 2011-2015 (Ruggles et al., 2015) converted to OES snapshot labor force totals for 2011 and 2016 (BLS, 2016b; BLS, 2016c).

The largest age group, 16-29, lost $0.52 \%$ of the total labor market (Figure 10). Presumably more workers aged into the 30-39 age group than children grew up to join the 16-29 group. This graduation from age group to age group can be seen in Figure 11, which illustrates the 40-49 age group lost more workers than aged up into the next group. By percentage, $2.48 \%$ of the labor force left the $40-49$ age group; only $1.84 \%$ (1.18\% increase in 50-64 age group, and 0.66\% increase in 65 and over age group) aged up. Two possible scenarios may explain the missing $0.64 \%$ of the labor market.

If all migration between age groups assumed no outside influence, that all workers remained in South Dakota, then all changes must equal the net increase of 24,860 workers between data sets (Table 11). Working backward, the increase of 7,991 in the 65 and over age group would come directly from the 50-64 age group. The 50-64 age group would receive its increase of 18,967 workers from the 40-49 group, to offset its contribution to the next group but increase by the amount in Table 10. To account for its loss and 2016 population, the 40-49 age group received 13,692 workers from the 30-39 group. The 30-39 age group must have received 23,096 from the 16-29 group. These movements would require 28,927 new workers to enter the 16-29 age group by 2016 (Table 11).

The second alternative would be workers leaving the state for better employment opportunities. As described above, the 40-49 age group are generally the experts in their fields. If they are emigrating to more lucrative economies, South Dakota's skilled resource pool will also deplete. 


\section{Table 10}

\section{Workers by Age Group and Proportion of Work Force}

\begin{tabular}{|c|c|c|c|c|c|c|c|}
\hline & & \multicolumn{6}{|c|}{ 16-29 } \\
\hline & & \multicolumn{2}{|c|}{ Number of Workers } & & \multicolumn{3}{|c|}{$\%$ of All Workers } \\
\hline & & 2011 & 2016 & & 2011 & 2016 & Change \\
\hline Low Wage & & 85,132 & 88,019 & 2,887 & $21.68 \%$ & $21.08 \%$ & $-0.60 \%$ \\
\hline Household Level & & 119,606 & 123,321 & 3,715 & $30.46 \%$ & $29.54 \%$ & $-0.92 \%$ \\
\hline \multirow[t]{5}{*}{ Higher Wage } & & 7,040 & 9,156 & 2,116 & $1.79 \%$ & $2.19 \%$ & $0.40 \%$ \\
\hline & Totals & 126,646 & 132,477 & 5,830 & $32.25 \%$ & $31.73 \%$ & $-0.52 \%$ \\
\hline & & \multicolumn{6}{|c|}{ 30-39 } \\
\hline & & \multicolumn{3}{|c|}{ Number of Workers } & \multicolumn{3}{|c|}{$\%$ of All Workers } \\
\hline & & 2011 & 2016 & & 2011 & 2016 & Change \\
\hline Low Wage & & 19,476 & 23,847 & 4,371 & $4.96 \%$ & $5.71 \%$ & $0.75 \%$ \\
\hline Household Level & & 52,007 & 60,061 & 8,138 & $13.24 \%$ & $14.38 \%$ & $1.14 \%$ \\
\hline \multirow[t]{5}{*}{ Higher Wage } & & 20,278 & 21,628 & 1,266 & $5.16 \%$ & $5.18 \%$ & $0.02 \%$ \\
\hline & Totals & 72,285 & 81,689 & 9,404 & $18.41 \%$ * & $19.56 \%$ & $1.16 \%$ \\
\hline & & \multicolumn{6}{|c|}{$40-49$} \\
\hline & & \multicolumn{2}{|c|}{ Number of Workers } & & \multicolumn{3}{|c|}{$\%$ of All Workers } \\
\hline & & 2011 & 2016 & & 2011 & 2016 & Change \\
\hline Low Wage & & 19,961 & 18,305 & $-1,656$ & $5.08 \%$ & $4.38 \%$ & $-0.70 \%$ \\
\hline Household Level & & 54,973 & 49,381 & $-5,562$ & $13.99 \% *$ & $11.83 \%$ & $-2.16 \% *$ \\
\hline \multirow[t]{5}{*}{ Higher Wage } & & 25,367 & 25,684 & 304 & $6.46 \%$ & $6.15 \%$ & $-0.31 \%$ \\
\hline & Totals & 80,340 & 75,065 & $-5,258$ & $20.46 \%$ & $17.98 \%$ & $-2.48 \%$ \\
\hline & & \multicolumn{6}{|c|}{$50-64$} \\
\hline & & \multicolumn{2}{|c|}{ Number of Workers } & & \multicolumn{3}{|c|}{$\%$ of All Workers } \\
\hline & & 2011 & 2016 & & 2011 & 2016 & Change \\
\hline Low Wage & & 27,734 & 29,675 & 1,941 & $7.06 \%$ & $7.11 \%$ & $0.04 \% *$ \\
\hline Household Level & & 66,404 & 72,373 & 5,969 & $16.91 \%$ & $17.33 \%$ & $0.43 \% *$ \\
\hline \multirow[t]{5}{*}{ Higher Wage } & & 29,166 & 34,173 & 5,007 & $7.43 \%$ & $8.18 \%$ & $0.75 \%$ \\
\hline & Totals & 95,570 & 106,546 & 10,976 & $24.33 \% *$ & $25.52 \%$ & $1.18 \% *$ \\
\hline & & \multicolumn{6}{|c|}{$65+$} \\
\hline & & \multicolumn{2}{|c|}{ Number of Workers } & & \multicolumn{3}{|c|}{$\%$ of All Workers } \\
\hline & & 2011 & 2016 & & 2011 & 2016 & Change \\
\hline Low Wage & & 11,597 & 12,359 & 737 & $2.95 \%$ & $2.96 \%$ & $0.01 \%$ \\
\hline Household Level & & 15,440 & 17,706 & 2,266 & $3.93 \%$ & $4.24 \%$ & $0.31 \%$ \\
\hline \multirow[t]{2}{*}{ Higher Wage } & & 2,399 & 4,058 & 5,725 & $0.61 \%$ & $0.97 \%$ & $0.36 \%$ \\
\hline & Totals & 17,839 & 21,764 & 7,991 & $4.55 \% *$ & $5.21 \%$ & $0.66 \% *$ \\
\hline
\end{tabular}

Note: * denotes error due to rounding. Calculations carried forward from Excel pivot table. Source: IPUMS ACS 5-yr data for periods 2006-2010 and 2011-2015 (Ruggles et al., 2015) converted to OES snapshot labor force totals for 2011 and 2016 (BLS, 2016a). 

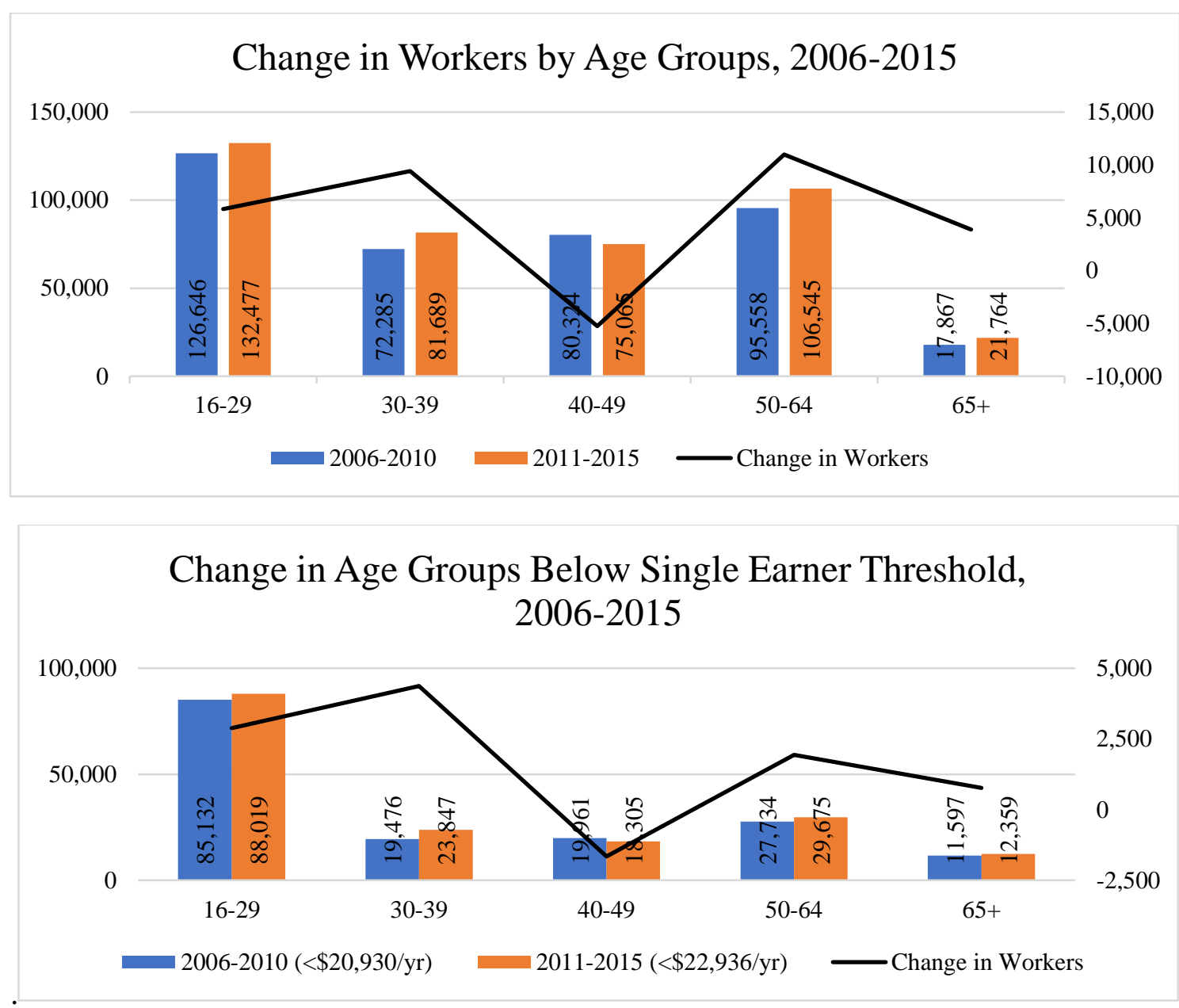

Figure 11. Change in all workers by age groups and below single earner threshold, 2006-2015. Source: IPUMS ACS 5-yr data for periods 2006-2010 and 2011-2015 (Ruggles et al., 2015) converted to OES snapshot labor force totals for 2011 and 2016 (BLS, 2016a). 
Table 11

Workers by Age under Single Earner and Household FESS Thresholds

\begin{tabular}{lcccccc}
\hline \multicolumn{7}{c}{ All Workers } \\
\hline AGE GROUPS & $\mathbf{2 0 0 6 - 2 0 1 0}$ & $\begin{array}{c}\text { Percent of All } \\
\text { Workers }\end{array}$ & $\mathbf{2 0 1 1 - 2 0 1 5}$ & $\begin{array}{c}\text { Percent of All } \\
\text { Workers }\end{array}$ & $\begin{array}{c}\text { Change in } \\
\text { Workers }\end{array}$ & $\begin{array}{c}\text { Percent } \\
\text { Change }\end{array}$ \\
\hline $16-29$ & 126,646 & $32.25 \%$ & 132,477 & $31.73 \%$ & $5,830^{*}$ & $-0.52 \%$ \\
$30-39$ & 72,285 & $18.41 \%$ & 81,689 & $19.56 \%$ & 9,404 & $1.15 \%$ \\
$40-49$ & 80,340 & $20.46 \%$ & 75,065 & $17.98 \%$ & $-5,275$ & $-2.48 \%$ \\
$50-64$ & 95,570 & $24.34 \%$ & 106,545 & $24.54 \%$ & 10,975 & $0.21 \% *$ \\
$65+$ & 17,839 & $4.55 \%$ & 21,764 & $6.19 \%$ & 3,925 & $1.64 \% *$ \\
\hline Totals & $\mathbf{3 9 2 , 6 8 0}$ & $100 \%$ & $\mathbf{4 1 7 , 5 4 0}$ & $100 \%$ & $\mathbf{2 4 , 8 5 9}$ & \\
\hline
\end{tabular}

Low Wage Earners

\begin{tabular}{|c|c|c|c|c|c|c|}
\hline AGE GROUPS & $\begin{array}{c}\mathbf{2 0 0 6 - 2 0 1 0} \\
(<\$ 20,930 / \mathrm{yr})\end{array}$ & $\begin{array}{c}\text { Percent of Low } \\
\text { Wage Earners }\end{array}$ & $\begin{array}{c}\mathbf{2 0 1 1 - 2 0 1 5} \\
(<\$ 22,936 / \mathrm{yr})\end{array}$ & $\begin{array}{c}\text { Percent of Low } \\
\text { Wage Earners }\end{array}$ & $\begin{array}{c}\text { Change in } \\
\text { Workers }\end{array}$ & $\begin{array}{l}\text { Percent } \\
\text { Change }\end{array}$ \\
\hline $16-29$ & 85,132 & $51.94 \%$ & 88,019 & $51.11 \%$ & 2,887 & $-0.83 \%$ \\
\hline $30-39$ & 19,476 & $11.88 \%$ & 23,847 & $13.85 \%$ & 4,371 & $1.97 \%$ \\
\hline $40-49$ & 19,961 & $12.18 \%$ & 18,305 & $10.63 \%$ & $-1,656$ & $-1.55 \%$ \\
\hline $50-64$ & 27,734 & $16.92 \%$ & 29,675 & $17.23 \%$ & $1,940 *$ & $0.31 \%$ \\
\hline $65+$ & 11,597 & $7.08 \%$ & 12,359 & $7.18 \%$ & 762 & $0.10 \%$ \\
\hline Totals & 163,900 & $100 \%$ & 172,204 & $100 \%$ & 8,304 & \\
\hline \multicolumn{7}{|c|}{ Less than Household Level Earners } \\
\hline AGE GROUPS & $\begin{array}{c}\mathbf{2 0 0 6 - 2 0 1 0} \\
(<\$ 41,860 / \mathrm{yr})\end{array}$ & $\begin{array}{c}\text { Percent of } \\
\text { Household } \\
\text { Level Earners }\end{array}$ & $\begin{array}{c}\mathbf{2 0 1 1 - 2 0 1 5} \\
(<\$ 45,873 / \mathrm{yr})\end{array}$ & $\begin{array}{c}\text { Percent of } \\
\text { Household } \\
\text { Level Earners }\end{array}$ & $\begin{array}{c}\text { Change in } \\
\text { Workers }\end{array}$ & $\begin{array}{l}\text { Percent } \\
\text { Change }\end{array}$ \\
\hline $16-29$ & 119,606 & $38.80 \%$ & 123,321 & $38.20 \%$ & 3,715 & $-0.60 \%$ \\
\hline $30-39$ & 52,007 & $16.84 \% *$ & 60,061 & $18.60 \%$ & 8,054 & $1.76 \%$ \\
\hline $40-49$ & 54,973 & $17.82 \%$ & 49,381 & $15.30 \%$ & $-5,592$ & $-2.52 \%$ \\
\hline $50-64$ & 66,404 & $21.53 \%$ & 72,373 & $22.42 \%$ & 5,969 & $0.89 \%$ \\
\hline $65+$ & 15,440 & $5.01 \%$ & 17,706 & $5.48 \%$ & 2,266 & $0.47 \%$ \\
\hline Totals & 308,430* & $100.00 \%$ & 322,843 & $100 \%$ & 14,412 & \\
\hline
\end{tabular}

Note: * denotes error due to rounding. Calculations carried forward from Excel pivot table. Source: IPUMS ACS 5-yr data for periods 2006-2010 and 2011-2015 (Ruggles et al., 2015) converted to OES snapshot labor force totals for 2011 and 2016 (BLS, 2016a). 
The importance of using microdata, made available by IPUMS, can be seen in Figure 12. From 2011 to 2016, the number of workers in South Dakota had a net increase of 24,860 . The combined Under 40 age groups comprised $51.29 \%$ of the work force in 2016, up $0.63 \%$ since 2011 (Figure 12). The actual increase in workers Under 40 was 15,234 (Table 11), or $61.28 \%$ of all new workers. Workers Over 40 appeared to be reduced, dropping from $49.34 \%$ to $48.71 \%$ of the labor market (Figure 12). Looking to Figure 12 for aggregated proportions of over and under 40 does not accurately convey worker trends.

Table 11 shows the reduced number of Over 40 workers indicates few older workers are leaving the labor force. The aggregated decrease in the $O v e r 40$ group was due to a net loss of 5,258 workers in the 40-49 age group. Over 5,000 more workers aged into the 50-64 age group than aged from the 30-39 age group (Figure 11).
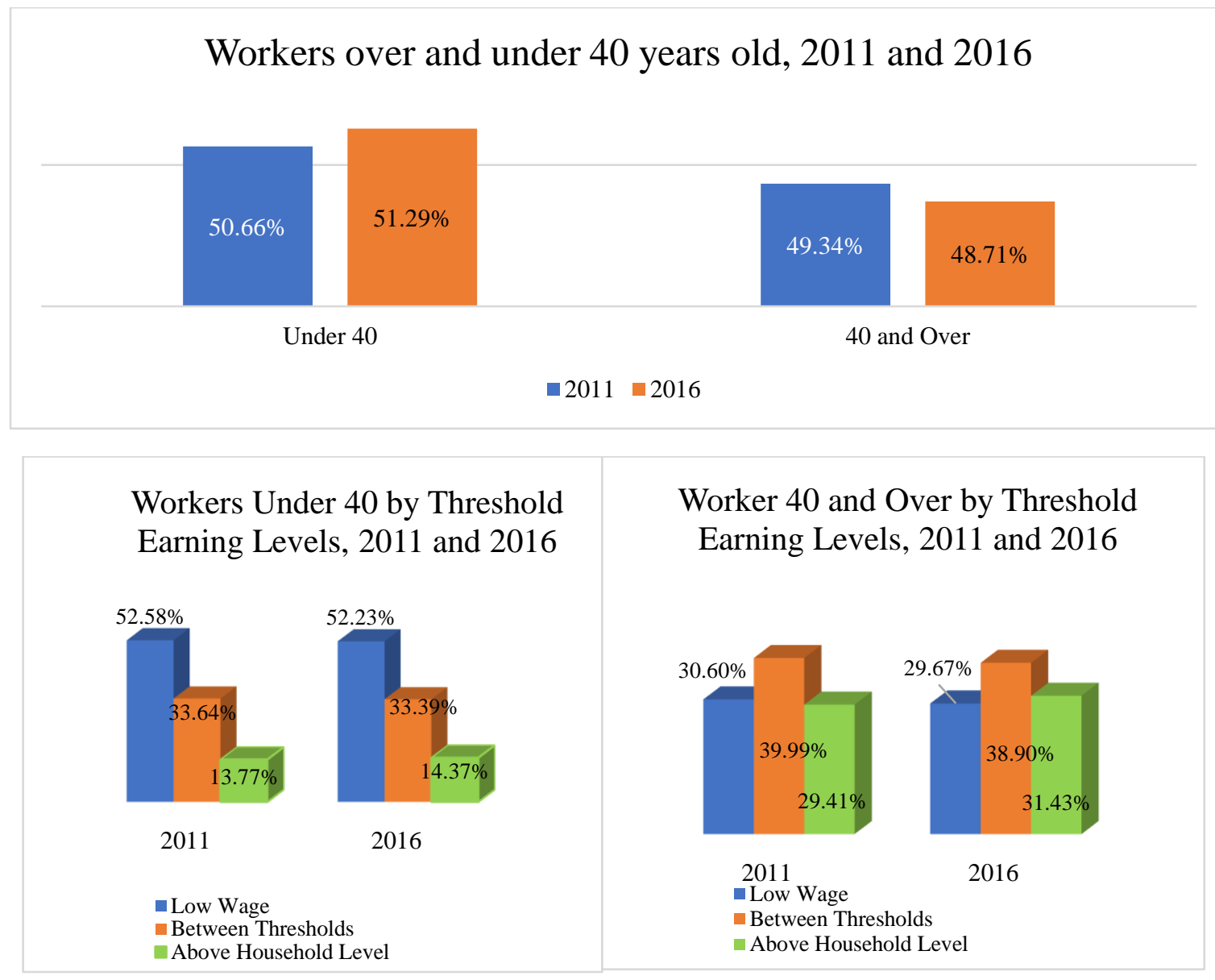

Figure 12. Workers over and under 40 years old and by threshold earning levels, 2011 and 2016. Source: IPUMS ACS 5-yr data for periods 2006-2010 and 2011-2015 (Ruggles et al., 2015) converted to OES snapshot labor force totals for 2011 and 2016 (BLS, 2016a).

DOI: $10.17605 / O S F . I O / Q F 4 H Y$ 


\section{National Comparison}

For 2016, South Dakota had an unemployment rate of 2.9\% (BLS, 2016b). The 2016 national unemployment rate averaged 4.9\%, 0.3\% higher than the average for 2007 (BLS, 2017). At least initially, South Dakota appears to have a thriving economy where nearly everyone willing to work can find a job. What is not clear is the quality of those jobs, nor whether other states have the same composition of labor markets. Using the FESS threshold, as applied to South Dakota, to evaluate the WA hourly wage and percentage of labor force for the other 37 markets reviewed by Pearce and Brooks should enable an informed comparison. Analysis followed six major activities.

Step One. FESS reports were accessed via the CWW website (http://selfsufficiencystandard.org/) for each state; each report provided a county by county calculation. Where multiple reports have been commissioned, such as New Jersey, the most recent report prior to the years of concern for this study were accessed. For example, New Jersey commissioned Pearce and Brooks to evaluate the state five times (1999, 2002, 2005, 2008, and 2011). The years of interest for this study's comparison were 2011 and 2016. Therefore, the 2011 report was accessed, and FESS values were recorded for each county into an Excel spreadsheet. U. S. Census American Fact Finder data provided the county level populations for each year in each state.

Step Two. Following a WA process, each county's population was weighted as a proportion of the state's total population. That weighted value was multiplied by the FESS value listed on the respective report. All counties' line item weighted wages were summed to yield a statewide minimum wage. In the case of 2011, the report was for one of the years desired, so it required no further data manipulation.

Step Three. For 2016 values, however, there were no New Jersey reports specific to that year. In fact, there were no reports done after 2011. As a result, the CPI calculator was used to bring each county's FESS value from 2011 to 2016, factoring in only the increase of inflation and 2016 populations for weighting. A statewide minimum wage was generated by following the same weighting process, but with the inflated hourly values.

Step Four. With an hourly threshold wage calculated, the same formula used by Pearce and Brooks (2000) was applied for annual household totals. In 2011, New Jersey's weighted value was $\$ 16.30$ per hour for a single earner within a two-earner household. This means it would take two adults working full-time to produce a minimum of $\$ 68,851$ per year $(16.30 \times 8$ hrs/day x 22 days/month x 12 months $x 2$ adults) to avoid using subsidies. Increasing each county value by inflation before weighting equaled $\$ 17.54$ per hour per worker for 2016 , or $\$ 74,089$ annually per household.

Step Five. Two separate IPUMS extracts were created for each state, ACS 5-year for 2011-2015 and ACS 5-year for 2006-2010 (Ruggles et al., 2015). The data sets were limited to values of EMPSTAT = 1, employed persons regardless of class of worker. Once extracted, each 
download was imported into Excel. The only line items excluded were those with OCC2010 codes in the 9800s, military personnel.

Step Six. A column for frequency (person number x person weight) was added to get a population count. Each extract was organized by earned income in ascending order. Frequency subtotals established the number of workers with annual incomes below that state's FESS hourly wage for a full-time worker, and for the number of workers with annual incomes sufficient to meet the entire family's need. Frequency was totaled for the whole labor force, enabling percentages to be established for each low wage threshold. This process was repeated for all states evaluated by FESS researchers.

In keeping with the New Jersey example, $49.99 \%$ of the state's labor market earned less than $\$ 34,426$ in 2011. Single earners below the FESS threshold of $\$ 37,045$ in 2016 reached $53.31 \%$. In this case, $3.32 \%$ more of the working population was potentially in need of subsidies to meet family basic needs by 2016 . Table 12 shows a ranking of all 38 labor markets by highest concentration of low wage workers. Single worker threshold values were used, that of one full-time working adult in a two-working adult household, to rank percentages of labor force earning less than FESS minimum wage for the year. The highest percentage of low wage workers ranked at the top of the list. For comparison, percentages established in Step Six above were used to rank states in both 2011 and 2016.

To illustrate low wage jobs as a proportion of the labor market, Figure 13 conveys lower percentages as bright green, with color turning red as percentages increase. These concentrations were recorded for 2011 and 2016, with the net changes conveyed on the heat map. Overall, most states improved (lessened) the number of low paying jobs except for some eastern states (Figure 13). Figure 14 uses the second threshold value calculated in Step Six, workers earning enough to meet the needs of the household with one income; this heat map shows the percentage of improvement between 2011 and 2016. The lowest percentages of labor market earning less than household threshold level was $68.71 \%$ in 2011 and $67.81 \%$ in 2016; in both years, this highest paying market was Louisiana. Table 13 lists the 2011 and 2016 calculated FESS WA minimum wages. Appendix G details the author's analysis of low wage population concentrations from IPUMS extracts for each of the 38 markets. 
Table 12

States with the Highest Proportion of Low Wage Workers, using FESS Weighted Average Thresholds for 2011 and 2016

\begin{tabular}{|c|c|c|}
\hline 2011 & & 2016 \\
\hline California & 1 & Connecticut \\
\hline Utah & 2 & California \\
\hline New York & 3 & Utah \\
\hline Pennsylvania & 4 & New York \\
\hline Illinois & 5 & Florida \\
\hline Florida & 6 & New Jersey \\
\hline New Jersey & 7 & Colorado \\
\hline Massachusetts & 8 & North Carolina \\
\hline Arizona & 9 & Virginia \\
\hline Connecticut & 10 & Arizona \\
\hline Colorado & 11 & Oregon \\
\hline Maryland & 12 & Maryland \\
\hline Hawaii & 13 & Illinois \\
\hline Montana & 14 & Massachusetts \\
\hline Kentucky & 15 & Washington \\
\hline Washington & 16 & Hawaii \\
\hline Oregon & 17 & Indiana \\
\hline Ohio & 18 & Pennsylvania \\
\hline Nebraska & 19 & Ohio \\
\hline Wisconsin & 20 & Wisconsin \\
\hline Oklahoma & 21 & Kentucky \\
\hline Texas & 22 & Nevada \\
\hline Indiana & 23 & Montana \\
\hline Tennessee & 24 & Texas \\
\hline Virginia & 25 & Georgia \\
\hline Georgia & 26 & South Carolina \\
\hline South Carolina & 27 & Tennessee \\
\hline Alabama & 28 & Nebraska \\
\hline South Dakota & 29 & Alabama \\
\hline Missouri & 30 & Oklahoma \\
\hline Washington, D. C. & 31 & Missouri \\
\hline North Carolina & 32 & Wyoming \\
\hline Nevada & 33 & Delaware \\
\hline Delaware & 34 & South Dakota \\
\hline West Virginia & 35 & Mississippi \\
\hline Wyoming & 36 & West Virginia \\
\hline Mississippi & 37 & Louisiana \\
\hline Louisiana & 38 & Washington, D. C \\
\hline
\end{tabular}

Source: Author's analysis of weighted average FESS data from Center for Women's Welfare reports, at http://www.selfsufficiencystandard.org, and IPUMS ACS 5-yr data for periods 2006-2010 and 2011-2015 (Ruggles et al., 2015). 


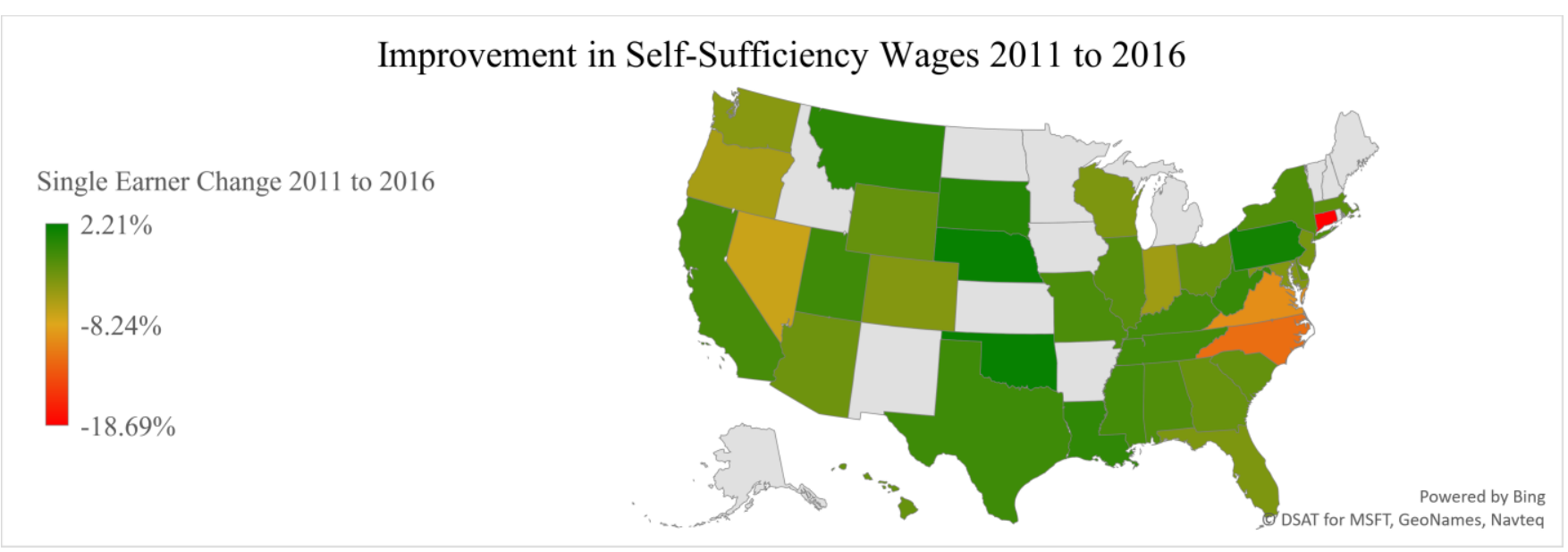

Figure 13. Improvement in work force composition earning less than single worker self-sufficiency wages, by state, from 2011 to 2016. Information represents author's analysis of weighted average FESS data from Center for Women's Welfare reports, at http://www.selfsufficiencystandard.org, and IPUMS USA ACS 5-yr data for periods 2006-2010 and 2011-2015 (Ruggles et al., 2015).

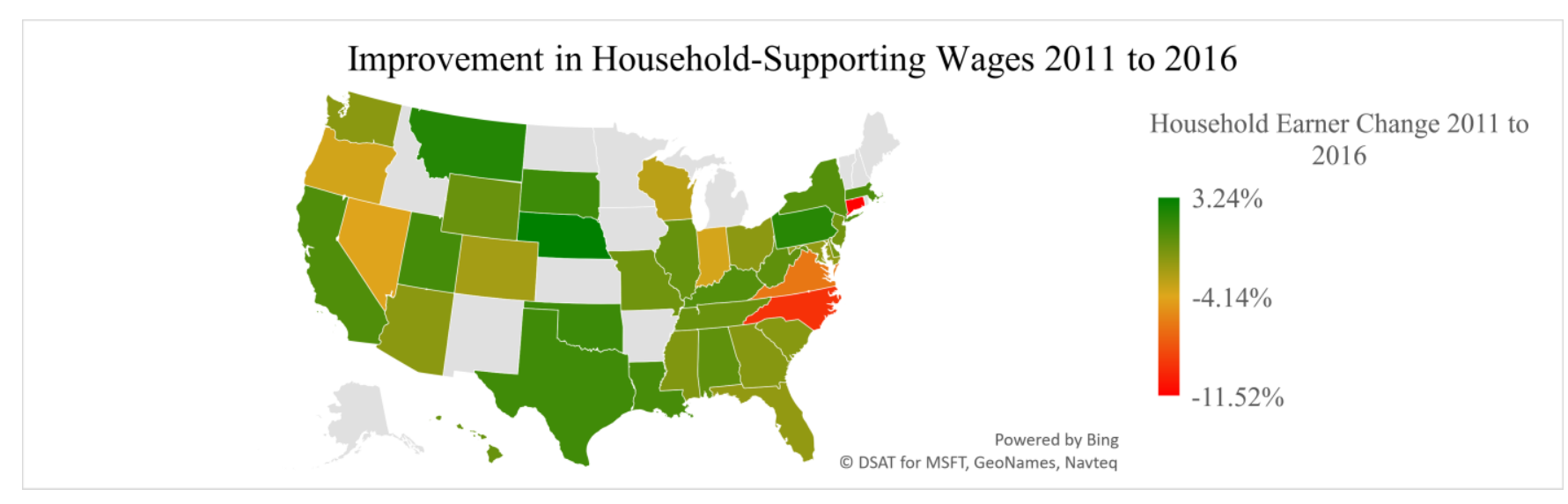

Figure 14. Improvement in work force composition earning less than household level self-sufficiency wages, by state, from 2011 to 2016. Information represents author's analysis of weighted average FESS data from Center for Women's Welfare reports, at http://www.selfsufficiencystandard.org, and IPUMS USA ACS 5-yr data for periods 2006-2010 and 2011-2015 (Ruggles et al., 2015).

DOI: 10.17605/OSF.IO/QF4HY 
Table 13

Weighted Averages of FESS Values for 38 States, 2011 and 2016

\begin{tabular}{|c|c|c|c|c|c|c|c|c|c|c|c|}
\hline \multirow[b]{2}{*}{ State } & \multicolumn{3}{|c|}{ FESS Cost of Living Data* } & \multicolumn{2}{|c|}{ FESS $2011^{*}$} & \multicolumn{2}{|c|}{ 5yr ACS 2006-2010*** } & \multicolumn{2}{|c|}{ FESS $2016^{* *}$} & \multicolumn{2}{|c|}{ 5yr ACS 2011-2015*** } \\
\hline & $\begin{array}{l}\text { Report } \\
\text { Year }\end{array}$ & $\begin{array}{l}\text { Hourly Per } \\
\text { Adult }\end{array}$ & $\begin{array}{l}\text { Household } \\
\text { Yearly }\end{array}$ & $\begin{array}{l}\text { Hourly Per } \\
\text { Adult }\end{array}$ & $\begin{array}{l}\text { Household } \\
\text { Yearly }\end{array}$ & $\begin{array}{l}\text { Below } \\
\text { Hourly } \\
\text { Threshold }\end{array}$ & $\begin{array}{c}\text { Below } \\
\text { Yearly } \\
\text { Threshold }\end{array}$ & $\begin{array}{c}\text { Hourly } \\
\text { Per Adult }\end{array}$ & $\begin{array}{l}\text { Household } \\
\text { Yearly }\end{array}$ & $\begin{array}{l}\text { Below } \\
\text { Hourly } \\
\text { Threshold }\end{array}$ & $\begin{array}{c}\text { Below } \\
\text { Yearly } \\
\text { Threshold }\end{array}$ \\
\hline Alabama & 2003 & 8.09 & 34,172 & 9.83 & 41,522 & $42.16 \%$ & $73.09 \%$ & 10.62 & 44,859 & $43.68 \%$ & $72.99 \%$ \\
\hline \multirow[t]{2}{*}{ Arizona } & 2002 & 10.29 & 43,465 & 12.78 & 53,983 & $49.51 \%$ & $80.75 \%$ & & & & \\
\hline & 2012 & 13.32 & 56,264 & & & & & 13.94 & 58,883 & $52.47 \%$ & $82.11 \%$ \\
\hline \multirow[t]{2}{*}{ California } & 2011 & 16.38 & 69,189 & 16.38 & 69,189 & $59.00 \%$ & $84.12 \%$ & & & & \\
\hline & 2014 & 16.61 & 70,161 & & & & & 16.82 & 71,048 & $60.10 \%$ & $83.51 \%$ \\
\hline \multirow[t]{2}{*}{ Colorado } & 2011 & 13.74 & 58,038 & 13.74 & 58,038 & $49.11 \%$ & $79.39 \%$ & & & & \\
\hline & 2015 & 15.29 & 64,585 & & & & & 15.49 & 65,430 & $53.10 \%$ & $81.57 \%$ \\
\hline \multirow[t]{2}{*}{ Connecticut } & 2005 & 13.82 & 58,376 & 15.95 & 67,373 & $49.32 \%$ & $79.39 \%$ & & & & \\
\hline & 2015 & 24.87 & 105,051 & & & & & 25.21 & 106,487 & $68.01 \%$ & $90.90 \%$ \\
\hline Delaware & 2003 & 9.43 & 39,832 & 11.36 & 47,985 & $40.28 \%$ & $72.39 \%$ & 12.18 & 51,448 & $42.64 \%$ & $73.05 \%$ \\
\hline Florida & 2007 & 11.26 & 47,562 & 12.26 & 51,786 & $49.99 \%$ & $81.64 \%$ & 13.19 & 55,715 & $53.64 \%$ & $83.23 \%$ \\
\hline Georgia & 2008 & 10.34 & 43,676 & 10.81 & 45,661 & $42.76 \%$ & $74.04 \%$ & 11.69 & 49,379 & $45.44 \%$ & $75.23 \%$ \\
\hline Hawaii & 2003 & 11.09 & 46,844 & 13.47 & 56,897 & $48.20 \%$ & $82.90 \%$ & 14.51 & 61,290 & $50.78 \%$ & $83.06 \%$ \\
\hline Illinois & 2009 & 12.93 & 54,16 & 13.51 & 57,066 & $50.13 \%$ & $79.55 \%$ & 14.55 & 61,459 & $52.00 \%$ & $79.68 \%$ \\
\hline \multirow[t]{2}{*}{ Indiana } & 2009 & 10.38 & 43,845 & 10.84 & 45,788 & $44.03 \%$ & $76.40 \%$ & & & & \\
\hline & 2016 & 12.54 & 52,969 & & & & & 12.54 & 52,969 & $49.29 \%$ & $80.17 \%$ \\
\hline Kentucky & 2001 & 8.96 & 37,847 & 11.30 & 47,731 & $47.67 \%$ & $79.45 \%$ & 12.04 & 50,857 & $48.60 \%$ & $79.02 \%$ \\
\hline Louisiana & 2003 & 7.68 & 32,440 & 9.30 & 39,325 & $39.22 \%$ & $68.71 \%$ & 9.80 & 41,395 & $39.25 \%$ & $67.81 \%$ \\
\hline \multirow[t]{2}{*}{ Maryland } & 2007 & 14.14 & 59,727 & 15.42 & 65,134 & $48.29 \%$ & $78.51 \%$ & & & & \\
\hline & 2016 & 17.54 & 74,089 & & & & & 17.54 & 74,089 & $52.24 \%$ & $80.14 \%$ \\
\hline Massachusetts & 2006 & 14.38 & 60,741 & 15.93 & 67,288 & $49.72 \%$ & $80.85 \%$ & 17.15 & 72,442 & $51.80 \%$ & $80.12 \%$ \\
\hline Mississippi & 2009 & 8.65 & 36,538 & 9.03 & 38,143 & $40.10 \%$ & $72.59 \%$ & 9.64 & 40,719 & $41.06 \%$ & $73.58 \%$ \\
\hline Missouri & 2002 & 7.97 & 33,665 & 9.98 & 42,156 & $41.61 \%$ & $73.40 \%$ & 10.79 & 45,577 & $42.97 \%$ & $73.86 \%$ \\
\hline Montana & 2008 & 10.39 & 43,8887 & 10.84 & 45,788 & $47.96 \%$ & $79.76 \%$ & 11.68 & 49,336 & $47.77 \%$ & $77.71 \%$ \\
\hline Nebraska & 2010 & 10.46 & 44,183 & 10.91 & 46,084 & $45.57 \%$ & $79.26 \%$ & 11.66 & 49,252 & $43.71 \%$ & $76.02 \%$ \\
\hline Nevada & 2002 & 9.34 & 39,452 & 11.63 & 49,125 & $40.74 \%$ & $76.25 \%$ & 12.52 & 52,884 & $47.94 \%$ & $80.51 \%$ \\
\hline New Jersey & 2011 & 16.30 & 68,851 & 16.30 & 68,851 & $49.99 \%$ & $79.24 \%$ & 17.54 & 74,089 & $53.31 \%$ & $79.78 \%$ \\
\hline New York & 2010 & 15.62 & 65,979 & 15.88 & 67,077 & $53.87 \%$ & $82.96 \%$ & 17.10 & 72,230 & $55.46 \%$ & $82.49 \%$ \\
\hline
\end{tabular}

(continued) 
Table 13

Weighted Averages of FESS Values for 38 States, 2011 and 2016 (continued)

\begin{tabular}{|c|c|c|c|c|c|c|c|c|c|c|c|}
\hline \multirow[b]{2}{*}{ State } & \multicolumn{3}{|c|}{ FESS Cost of Living Data* } & \multicolumn{2}{|c|}{ FESS $2011^{*}$} & \multicolumn{2}{|c|}{$5 \mathrm{yr}$ ACS $2006-2010 * * *$} & \multicolumn{2}{|c|}{ FESS $2016 * *$} & \multicolumn{2}{|c|}{ 5yr ACS 2011-2015*** } \\
\hline & $\begin{array}{c}\text { Report } \\
\text { Year }\end{array}$ & $\begin{array}{c}\text { Hourly Per } \\
\text { Adult }\end{array}$ & $\begin{array}{c}\text { Household } \\
\text { Yearly }\end{array}$ & $\begin{array}{l}\text { Hourly Per } \\
\text { Adult }\end{array}$ & $\begin{array}{l}\text { Household } \\
\text { Yearly }\end{array}$ & $\begin{array}{c}\text { Below } \\
\text { Hourly } \\
\text { Threshold }\end{array}$ & $\begin{array}{l}\text { Below } \\
\text { Yearly } \\
\text { Threshold }\end{array}$ & $\begin{array}{l}\text { Hourly } \\
\text { Per Adult }\end{array}$ & $\begin{array}{c}\text { Household } \\
\text { Yearly }\end{array}$ & $\begin{array}{l}\text { Below } \\
\text { Hourly } \\
\text { Threshold }\end{array}$ & $\begin{array}{l}\text { Below } \\
\text { Yearly } \\
\text { Threshold }\end{array}$ \\
\hline \multirow[t]{2}{*}{ North Carolina } & 1997 & 7.16 & 30,244 & 10.01 & 42,282 & $40.95 \%$ & $73.66 \%$ & & & & \\
\hline & 2017 & 13.92 & 58,798 & & & & & 13.58 & 57,362 & $52.73 \%$ & $83.03 \%$ \\
\hline \multirow[t]{2}{*}{ Ohio } & 2011 & 11.79 & 49,801 & 11.79 & 49,801 & $46.74 \%$ & $77.72 \%$ & & & & \\
\hline & 2015 & 12.73 & 53,772 & & & & & 12.93 & 54,616 & $49.17 \%$ & $79.11 \%$ \\
\hline Oklahoma & 2009 & 9.62 & 40,635 & 10.04 & 42,409 & $45.00 \%$ & $76.26 \%$ & 10.83 & 45,746 & $43.12 \%$ & $75.02 \%$ \\
\hline \multirow[t]{2}{*}{ Oregon } & 2011 & 11.47 & 48,449 & 11.47 & 48,449 & $46.83 \%$ & $76.68 \%$ & & & & \\
\hline & 2014 & 13.18 & 55,672 & & & & & 13.36 & 56,433 & $52.46 \%$ & $80.37 \%$ \\
\hline \multirow[t]{2}{*}{ Pennsylvania } & 2010 & 13.24 & 55,926 & 13.46 & 56,855 & $50.56 \%$ & $82.04 \%$ & & & & \\
\hline & 2012 & 13.56 & 57,277 & & & & & 14.20 & 59,981 & $49.23 \%$ & $80.12 \%$ \\
\hline South Carolina & 2016 & 11.03 & 46,591 & 10.21 & 43,127 & $42.60 \%$ & $75.53 \%$ & 11.03 & 46,591 & $45.06 \%$ & $76.77 \%$ \\
\hline South Dakota & 2000 & 7.67 & 32,398 & 9.91 & 41,860 & $41.74 \%$ & $78.55 \%$ & 10.86 & 45,873 & $41.24 \%$ & $77.32 \%$ \\
\hline Tennessee & 2002 & 8.22 & 34,721 & 10.25 & 43,296 & $43.59 \%$ & $76.06 \%$ & 11.05 & 46,675 & $44.52 \%$ & $76.32 \%$ \\
\hline Texas & 1996 & 7.11 & 30,033 & 10.48 & 44,268 & $44.82 \%$ & $73.80 \%$ & 11.15 & 47,098 & $45.52 \%$ & $72.68 \%$ \\
\hline Utah & 2001 & 10.37 & 43,803 & 13.43 & 56,728 & $57.68 \%$ & $85.18 \%$ & 14.28 & 60,319 & $58.41 \%$ & $84.21 \%$ \\
\hline \multirow[t]{2}{*}{ Virginia } & 2006 & 10.99 & 46,422 & 12.32 & 52,040 & $42.86 \%$ & $73.38 \%$ & & & & \\
\hline & 2012 & 15.32 & 64,712 & & & & & 16.08 & 67,922 & $52.66 \%$ & $79.65 \%$ \\
\hline \multirow[t]{2}{*}{ Washington } & 2011 & 13.36 & 56,433 & 13.36 & 56,433 & $46.99 \%$ & $77.65 \%$ & & & & \\
\hline & 2014 & 14.77 & 62,388 & & & & & 14.99 & 63,318 & $51.16 \%$ & $78.98 \%$ \\
\hline Washington D. C. & 2005 & 13.95 & 58,925 & 16.11 & 68,049 & $41.40 \%$ & $72.46 \%$ & 17.32 & 73,160 & $39.19 \%$ & $70.08 \%$ \\
\hline West Virginia & 2005 & 8.10 & 34,214 & 9.36 & 39,537 & $40.28 \%$ & $71.54 \%$ & 10.08 & 42,578 & $40.65 \%$ & $71.43 \%$ \\
\hline \multirow[t]{2}{*}{ Wisconsin } & 2004 & 9.85 & 41,606 & 11.73 & 49,548 & $45.24 \%$ & $77.72 \%$ & & & & \\
\hline & 2016 & 13.20 & 55,757 & & & & & 13.20 & 55,757 & $48.89 \%$ & $80.64 \%$ \\
\hline \multirow[t]{2}{*}{ Wyoming } & 2007 & 9.62 & 40,635 & 10.46 & 44,183 & $40.28 \%$ & $70.62 \%$ & & & & \\
\hline & 2016 & 12.01 & 50,730 & & & & & 12.01 & 50,730 & $42.82 \%$ & $70.84 \%$ \\
\hline
\end{tabular}

* Author's analysis of weighted average FESS data from Center for Women's Welfare reports, at http://www.selfsufficiencystandard.org. ** Where needed, values were updated using the CPI Inflation Calculator at https://data.bls.gov/cgi-bin/cpicalc.pl by converting most recent report estimations. *** IPUMS USA ACS 5-yr data for periods 2006-2010 and 2011-2015 (Ruggles et al., 2015).

DOI: $10.17605 / O S F . I O / Q F 4 H Y$ 


\section{RESULTS}

If economic strata were limited to three equivalently populated typologies, the lower wage tier would be composed of no more than one-third of all available jobs. IPUMS data (Ruggles et al., 2015) combined with highly conservative WA income threshold standards revealed 42.79\% of South Dakota's 2016 labor force earned less than the annual FESS value for a single earner (Table 4). Therefore, the state could be viewed as suffering from an elevated proportion of low income earners in the labor market. When compared to 37 other states, South Dakota was found to have a more balanced labor market (Table 12); the state had comparatively lower concentrations of low wage jobs than 35 other states. In fact, only one occupational category (Food Service) emerged as a guarantee to underemployment (Table 8). Food Service represents $9.92 \%$ of the labor market, indicating the remaining proportion of low wage jobs must consist of those with higher hourly wages but with less than full-time hours.

\section{South Dakota}

Investigating South Dakota's labor statistics, on a microdata level, revealed unexpected results. Initially, this researcher believed the predominance of low wage jobs in the Sioux Falls MSA was a symptom of a larger issue of precariousness for workers across the state. BLS data, however, revealed South Dakota had an unemployment rate 49\% lower than the national average for 2016; national unemployment averaged 4.9\% for 2016, while South Dakota averaged 2.9\% (BLS, 2017).

For 2016, 78.20\% (326,516/417,540) of all workers were employed full-time with $84.71 \%(276,597 / 326,516)$ of those workers employed year-round (Table 4). Sectors with low wage proportions greater than $42.79 \%$ would be considered to have an overrepresented concentration of low wage jobs. Although $68.23 \%$ of all low wage jobs were found in the private sector, low wage jobs only made up $38.41 \%$ of all private sector jobs (Table 4 ). This can be explained in part where only $20.43 \%$ of all low wage earners worked in the public sector (government/nonprofit), yet $56.48 \%$ of all public-sector jobs were low wage. Similarly, $10.77 \%$ of all low wage earners were self-employed; of those identifying as self-employed, 55.93\% earned a low wage income (Table 4). Table 5 shows marginally higher values for each sector in 2011.

Comparing Table 4 and Table 5 confirms each sector reduced its proportion of low wage jobs by approximately one percent over the five years between data sets. One area of note is the change in unpaid family workers from 2011 to 2016 . Approximately $9.8 \%$ more people opted to stay at home to care for the family, indicating there were 91 more people doing so in 2016 . The proportion of family workers considered low wage earners, even though the name indicates unpaid, remarkably increased from $51.38 \%$ to $90.34 \%$.

As with the various sectors, each occupational category was evaluated for concentration

of low wage earners (Table 6). Occupations with an overrepresentation of low wage jobs would have more than $42.79 \%$ in 2016 and $43.76 \%$ in 2011 qualified as such. Reviewing Table 6 and

DOI: 10.17605/OSF.IO/QF4HY 
Table 7 identifies seven of the 22 categories with above average proportions of low wage jobs (Table 14).

Table 14

Occupations with Overrepresented Low Wage Concentrations

\begin{tabular}{llll}
\multicolumn{1}{c}{ Occupation } & 2016 & Change & 2011 \\
\hline Food preparation and serving related & $89.95 \%$ & $+2.23 \%$ & $87.72 \%$ \\
Building, grounds cleaning, and maintenance & $70.93 \%$ & $-1.22 \%$ & $72.15 \%$ \\
Personal care and service & $66.06 \%$ & $-5.25 \%$ & $71.31 \%$ \\
Healthcare support & $62.29 \%$ & $+0.11 \%$ & $62.18 \%$ \\
Sales and related & $49.05 \%$ & $-0.71 \%$ & $49.76 \%$ \\
Arts, design, entertainment, sports and media & $48.21 \%$ & $-4.07 \%$ & $52.28 \%$ \\
Farming, fishing, and forestry & $44.13 \%$ & $-21.23 \%$ & $65.36 \%$ \\
\hline
\end{tabular}

Both snapshot data (U. S. Census, 2016a; U. S. Census, 2016b) and IPUMS data (Ruggles et al., 2015) were products of U. S. Census ACS information; they differed slightly due to snapshots, such as Tables DP03 (Appendix B) and S2303 (Appendix C), provided estimates for specific time periods, while IPUMS data listed every component line item. Using exact proportions derived from microdata, snapshots were dissected for more detailed analysis. Microdata using WA values for FESS indicated $41.24 \%$ of South Dakota's labor force earned less than $\$ 22,936$ in 2016 (Appendix G); Table 4, using summative values for age group populations within each occupation confirmed the same percentage. while Table 6 revealed $42.79 \%$ of total workers were low wage earners. The $1.55 \%$ discrepancy likely was due to the differences in OES snapshot totals, indicating low wage earners totaled a slightly higher percentage than when part of the much larger IPUMS data set.

When examining workers by age, microdata revealed increases in workers at both ends of the spectrum. Between ACS 5-year data for 2006-2010 and ACS -year data for 2011-2015, new workers (ages 16-29) did enter the work force. Approximately 5,830 new workers had jobs in 2016 (Table 11). In general, it is not surprising for younger workers to earn less than their older counterparts. Figure 12 shows $52.23 \%$ of all workers under 40 earned less than the FESS single earner threshold (\$22,936/year), and 85.62\% earned less than the household threshold (\$45,873/year).

On the other end of the spectrum, workers age 50 or more increased 14,884 between data sets (Table 11). The 40-49 age group had a net decline of 5,258 workers. Older workers may have greater numbers of Higher Wage earners than younger workers, but those still working appear to need the income. In 2016, 29.67\% of all workers over 40 did not earn $\$ 22,936$ per year; $68.57 \%$ of older workers did not earn the household threshold level of $\$ 45,873$ per year.

DOI: $10.17605 / O S F . I O / Q F 4 H Y$ 
Discrepancies. One issue of relevance is the OES snapshot data are figures reported by employers, while ACS data are self-reported by employees. When trying to determine age groups as proportions of the labor force and individual occupations, the extreme detail in IPUMS data revealed altered occupational totals. Initially, Tables 6 through 9 were calculated based upon OES values. Upon engaging in further analysis of the microdata to identify age groups within each occupation, and as a percentage of the overall work force, ACS self-reporting shifted approximately $10 \%$ of workers to a different category. Using Table 6 to test the values, prior occupational totals were replaced with cumulative counts from each of five age groups. These groups were (a) 16-29 years of age, (b) 30-39 years of age, (c) 40-49 years of age, (d) 50-64 years of age, and (e) 65 years of age and over.

Interestingly, most occupations had only minor percentage changes, with Community and Social Services $(1.57 \%)$ and Protection Service (1.55\%) remaining exactly the same. The drastic difference was in the Management category. Previously reported by OES, Management represented $2.77 \%$ (11,580) of 417,540 workers in 2016 (BLS, 2016b). Contrary to employer classification, the detailed ACS reported data from IPUMS showed $10.74 \%(44,862)$ of all workers identified as managers (Ruggles et al., 2015). Two other changes are notable.

Food Service changed from $9.92 \%$ (41,410 workers) to $7.13 \%$ (29,790 workers) of the work force. OES data indicated Farming, Fishing, and Forestry had 1,630 workers $(0.39 \%)$ in 2016.; IPUMS microdata revealed 9,793 workers, representing $2.35 \%$ of the work force, identified with this occupation. By applying the previously determined low wage proportions to each occupation, a total Low Wage work force changed from 178,680 to 172,209. The new value for low wage workers yielded a proportion of the work force of $41.24 \%$, consistent with FESS WA microdata analysis in Appendix G, and removed a prior discrepancy of $1.55 \%$.

A possible reason for the inconsistency between reporting methods (OES and ACS) may be turnover. Either each of the 11,580 managers reported by employers changed jobs almost annually, to yield four times as many workers over five years, or employees gravitate toward identifying with a management position regardless of their industry. Whereas employers may be more discriminating as to which jobs they recognize as managerial, employers perhaps limit them to executive positions. The possibility exists for many Food Service managers to avoid being categorized as in that industry to concentrate on their roles as leaders.

One additional calculation was required when applying ACS 5-year microdata to OES snapshot data, such as Table 6 and Table 7. Total workers in 2016 were 417,540 in OES and 695,195 in ACS; total workers in 2011 were 392,680 in OES and 655,173 in ACS. OES snapshots were $60 \%$ of ACS totals. To keep proportions intact, each ACS 5-year 2011-2015 value was multiplied by 60.0608462373866\%; each ACS 5-year 2006-2010 value was multiplied by $59.9353147947183 \%$. These constants represent the exact ratios applicable to each dataset. 
Table 15

Occupational Analysis with Age Group Proportions Applied, 2016

\begin{tabular}{|c|c|c|c|c|c|}
\hline \multirow[b]{2}{*}{ Occupation } & \multicolumn{2}{|c|}{$\begin{array}{c}\text { All Workers } \\
\text { (OES wage data) }\end{array}$} & \multicolumn{3}{|c|}{$\begin{array}{c}\text { Low Wage Earners } \\
\text { (below } \$ 10.86 / \mathrm{hr}, \text { FESS per earner) }\end{array}$} \\
\hline & $\begin{array}{c}\text { Workers in } \\
\text { category }\end{array}$ & $\begin{array}{l}\text { Percent of All } \\
\text { workers }\end{array}$ & $\begin{array}{c}\text { Low wage } \\
\text { workers }\end{array}$ & $\begin{array}{l}\text { Percent of All low } \\
\text { wage workers }\end{array}$ & $\begin{array}{l}\text { Percent of } \\
\text { occupation }\end{array}$ \\
\hline Management & 44,862 & $10.74 \%$ & 10,107 & $5.87 \%$ & $22.53 \%$ \\
\hline $\begin{array}{l}\text { Business \& Financial } \\
\text { Operations }\end{array}$ & 12,710 & $3.04 \%$ & 1,909 & $1.11 \%$ & $15.02 \%$ \\
\hline Computer \& Mathematical & 5,303 & $1.27 \%$ & 700 & $0.41 \%$ & $13.20 \%$ \\
\hline $\begin{array}{l}\text { Architecture \& } \\
\text { Engineering }\end{array}$ & 4,718 & $1.13 \%$ & 492 & $0.29 \%$ & $10.43 \%$ \\
\hline $\begin{array}{l}\text { Life, Physical, \& Social } \\
\text { Sciences }\end{array}$ & 3,080 & $0.74 \%$ & 534 & $0.31 \%$ & $17.32 \%$ \\
\hline $\begin{array}{l}\text { Community \& Social } \\
\text { Service }\end{array}$ & 6,568 & $1.57 \%$ & 1,567 & $0.91 \%$ & $23.86 \%$ \\
\hline Legal & 2,416 & $0.58 \%$ & 471 & $0.27 \%$ & $19.49 \%$ \\
\hline $\begin{array}{l}\text { Education, Training \& } \\
\text { Library }\end{array}$ & 21,305 & $5.10 \%$ & 7,864 & $4.57 \%$ & $36.91 \%$ \\
\hline $\begin{array}{l}\text { Arts, Design, } \\
\text { Entertainment, Sports, \& } \\
\text { Media }\end{array}$ & 5,575 & $1.34 \%$ & 2,688 & $1.56 \%$ & $48.21 \%$ \\
\hline $\begin{array}{l}\text { Healthcare Practitioners \& } \\
\text { Technical }\end{array}$ & $24,18 \mathrm{re} 7$ & $5.79 \%$ & 3,785 & $2.20 \%$ & $15.65 \%$ \\
\hline Healthcare Support & 10,858 & $2.60 \%$ & 6,763 & $3.93 \%$ & $62.29 \%$ \\
\hline Protective Service & 6,462 & $1.55 \%$ & 2,275 & $1.32 \%$ & $35.21 \%$ \\
\hline $\begin{array}{l}\text { Food Preparation \& } \\
\text { Serving Related }\end{array}$ & 29,790 & $7.13 \%$ & 26,796 & $15.56 \%$ & $89.95 \%$ \\
\hline $\begin{array}{l}\text { Building \& Grounds } \\
\text { Cleaning \& Maintenance }\end{array}$ & 16,633 & $3.98 \%$ & 11,798 & $6.85 \%$ & $70.93 \%$ \\
\hline Personal Care \& Service & 17,404 & $4.17 \%$ & 11,497 & $6.68 \%$ & $66.06 \%$ \\
\hline Sales \& Related & 44,040 & $10.55 \%$ & 21,602 & $12.54 \%$ & $49.05 \%$ \\
\hline $\begin{array}{l}\text { Office \& Administrative } \\
\text { Support }\end{array}$ & 59,364 & $14.22 \%$ & 24,678 & $14.33 \%$ & $41.57 \%$ \\
\hline $\begin{array}{l}\text { Farming, Fishing, \& } \\
\text { Forestry }\end{array}$ & 9,793 & $2.35 \%$ & 4,322 & $2.51 \%$ & $44.13 \%$ \\
\hline Construction \& Extraction & 24,567 & $5.88 \%$ & 8,864 & $5.15 \%$ & $36.08 \%$ \\
\hline $\begin{array}{l}\text { Installation, Maintenance, } \\
\text { \& Repair }\end{array}$ & 13,204 & $3.16 \%$ & 2,810 & $1.63 \%$ & $21.28 \%$ \\
\hline Production & 28,042 & $6.72 \%$ & 10,045 & $5.83 \%$ & $35.82 \%$ \\
\hline $\begin{array}{l}\text { Transportation \& Material } \\
\text { Moving }\end{array}$ & 26,659 & $6.38 \%$ & 10,642 & $6.18 \%$ & $39.92 \%$ \\
\hline TOTAL & 417,540 & $100.00 \% *$ & 172,209 & $100.01 \% *$ & $41.24 \%$ \\
\hline
\end{tabular}

Source: Proportions of each category established by analysis of IPUMS USA (Ruggles et al., 2015) data for 5-year ACS 2011-2015, dissected by age group within each occupation, then applied to OES snapshots for occupational categories (BLS, 2016b).

(1) Estimates for detailed occupations do not sum to the totals because the totals include occupations not shown separately. Estimates did not include self-employed workers, but low wage proportions did factor in this sector.

(2) Occupations with low wage percentages above $41.24 \%$ indicate low wage workers are over-represented in that industry.

(3) Reconfiguration of Table 6.

DOI: 10.17605/OSF.IO/QF4HY 
Table 16

Occupational Typologies using Age Group Proportions, 2016

\begin{tabular}{|c|c|c|c|c|c|}
\hline $\begin{array}{c}\text { Occupational } \\
\text { Typology }\end{array}$ & Occupational Title & \multirow[t]{2}{*}{$\begin{array}{l}\text { Number } \\
\text { Employed }\end{array}$} & \multirow[t]{2}{*}{$\begin{array}{l}\text { Percent of } \\
\text { Total } \\
\text { Employment }\end{array}$} & \multirow[t]{2}{*}{$\begin{array}{c}\text { Median } \\
\text { Hourly } \\
\text { Wage }\end{array}$} & \multirow[t]{2}{*}{$\begin{array}{c}2 / 3 \\
\text { Median }\end{array}$} \\
\hline FESS thresho & lue for $2016=\$ 10.86 /$ hour & & & & \\
\hline \multirow{2}{*}{$\begin{array}{ll}0 \\
3 \\
0 \\
3 & 5 \\
3 & 3 \\
3\end{array}$} & Food Preparation/Serving & 29,790 & $7.13 \%$ & \multirow[t]{2}{*}{$\$ 9.62$} & \\
\hline & Low wage total & 29,790 & $7.13 \%$ & & \\
\hline \multirow{10}{*}{ 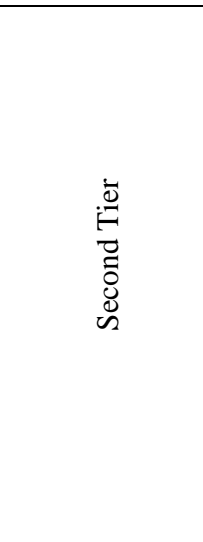 } & Personal Care & 17,404 & $4.17 \%$ & $\$ 10.97$ & $\$ 7.31$ \\
\hline & Building/Grounds Services & 16,633 & $3.98 \%$ & $\$ 11.25$ & $\$ 7.50$ \\
\hline & Sales & 44,040 & $10.55 \%$ & $\$ 12.49$ & $\$ 8.33$ \\
\hline & $\begin{array}{l}\text { Farming, Fishing, \& } \\
\text { Forestry }\end{array}$ & 9,793 & $2.35 \%$ & $\$ 12.52$ & $\$ 8.35$ \\
\hline & Healthcare Support & 10,858 & $2.60 \%$ & $\$ 12.63$ & $\$ 8.42$ \\
\hline & $\begin{array}{l}\text { Office \& Administrative } \\
\text { Support }\end{array}$ & 59,364 & $14.22 \%$ & $\$ 14.00$ & $\$ 9.33$ \\
\hline & $\begin{array}{l}\text { Transportation \& Material } \\
\text { Moving }\end{array}$ & 26,659 & $6.38 \%$ & $\$ 14.06$ & $\$ 9.37$ \\
\hline & Production & 28,042 & $6.72 \%$ & $\$ 14.98$ & $\$ 9.99$ \\
\hline & Arts/Entertainment & 5,575 & $1.34 \%$ & $\$ 16.15$ & $\$ 10.77$ \\
\hline & Second Tier total & 218,368 & $52.31 \%$ & & \\
\hline \multirow{14}{*}{ 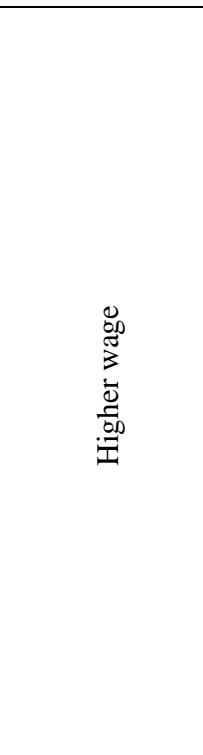 } & Construction \& Extraction & 24,567 & $5.88 \%$ & $\$ 17.13$ & $\$ 11.42$ \\
\hline & Protective Service & 6,462 & $1.55 \%$ & $\$ 17.21$ & $\$ 11.47$ \\
\hline & $\begin{array}{l}\text { Community \& Social } \\
\text { Service }\end{array}$ & 6,568 & $1.57 \%$ & $\$ 18.26$ & $\$ 12.17$ \\
\hline & $\begin{array}{l}\text { Education, Training, \& } \\
\text { Library }\end{array}$ & 21,305 & $5.10 \%$ & $\$ 18.66$ & $\$ 12.44$ \\
\hline & $\begin{array}{l}\text { Installation, Maintenance \& } \\
\text { Repair }\end{array}$ & 13,204 & $3.16 \%$ & $\$ 19.67$ & $\$ 13.11$ \\
\hline & $\begin{array}{l}\text { Life, Physical, \& Social } \\
\text { Sciences }\end{array}$ & 3,080 & $0.74 \%$ & $\$ 24.03$ & $\$ 16.02$ \\
\hline & Healthcare Practitioners & 24,187 & $5.79 \%$ & $\$ 25.70$ & $\$ 17.13$ \\
\hline & Business \& Financial & 12,710 & $3.04 \%$ & $\$ 27.93$ & $\$ 18.62$ \\
\hline & Computer \& Mathematical & 5,303 & $1.27 \%$ & $\$ 28.63$ & $\$ 19.09$ \\
\hline & Legal & 2,416 & $0.58 \%$ & $\$ 28.76$ & $\$ 19.09$ \\
\hline & Architecture \& Engineering & 4,718 & $1.13 \%$ & $\$ 30.09$ & $\$ 20.06$ \\
\hline & Management & 44,862 & $10.74 \%$ & $\$ 44.14$ & $\$ 29.43$ \\
\hline & Higher wage total & 169,382 & $40.55 \%$ & & \\
\hline & All Jobs & 417,540 & $99.99 \% *$ & & \\
\hline
\end{tabular}

Source: Bureau of Labor Statistics. (2016b). Occupational Employment Statistics (OES) data tables. Multiple occupations for one geographical area, South Dakota, May 2016. Retrieved from https://data.bls.gov/oes/ and IPUMS data from Ruggles et al. (2015).

Reconfiguration of Table 8 .

DOI: $10.17605 / O S F . I O / Q F 4 H Y$ 
Occupations. Studying the occupational wage ranges in Figure 4 and Figure 5 for all 22 categories makes evident the seven occupations with the highest proportion of low wage jobs also have the smallest wage ranges, and median hourly wages below or near the FESS minimum wage. The single exception was Sales and Related; its wage range covers nearly $\$ 30.00$ from minimum to maximum compared to the $\$ 10.00$ to $\$ 20.00$ range of the other six occupations in Table 14. Farming, Fishing, and Forestry glaringly lost $21.23 \%$ of its work force from 2011 to 2016. Even with a huge industry loss, Farming, Fishing, and Forestry still had an overrepresented concentration of low income workers (Table 14).

When typologies were introduced and applied to the occupational categories, each of the seven occupations with high concentrations of low wage jobs appeared exclusively in Low Wage and Second Tier typologies (Table 8). Although Sales had the largest wage range, the category of Arts and Entertainment had the highest median wage among the seven. The reader may recall Arts and Entertainment was the only occupation to change its typology from 2006-2010 to 2011-2015, moving from Higher Wage to Second Tier. Table 6 and Table 7 provide detail as to number of workers employed and percentage of total employment; these values are restated for the seven occupations in Table 17.

Table 17

Overrepresented Low Wage Occupations as Part of the South Dakota Labor Market

\begin{tabular}{|c|c|c|c|c|}
\hline \multirow[b]{2}{*}{ Occupation } & \multicolumn{2}{|c|}{2016} & \multicolumn{2}{|c|}{2011} \\
\hline & $\begin{array}{l}\text { Number of } \\
\text { Employees }\end{array}$ & $\begin{array}{c}\text { Percent of Total } \\
\text { Employment }\end{array}$ & $\begin{array}{l}\text { Number of } \\
\text { Employees }\end{array}$ & $\begin{array}{l}\text { Percent of Total } \\
\text { Employment }\end{array}$ \\
\hline $\begin{array}{l}\text { Food preparation and } \\
\text { serving related }\end{array}$ & 41,410 & $9.92 \%$ & 38,590 & $9.83 \%$ \\
\hline $\begin{array}{l}\text { Building, grounds cleaning, } \\
\text { and maintenance }\end{array}$ & 16,780 & $4.02 \%$ & 15,790 & $4.02 \%$ \\
\hline Personal care and service & 14,320 & $3.43 \%$ & 12,320 & $3.14 \%$ \\
\hline Healthcare support & 10,540 & $2.52 \%$ & 10,840 & $2.76 \%$ \\
\hline Sales and related & 47,440 & $11.36 \%$ & 45,100 & $11.49 \%$ \\
\hline $\begin{array}{l}\text { Arts, design, entertainment, } \\
\text { sports and media }\end{array}$ & 5,560 & $1.33 \%$ & 5,530 & $1.41 \%$ \\
\hline $\begin{array}{l}\text { Farming, fishing, and } \\
\text { forestry }\end{array}$ & 1,630 & $0.39 \%$ & 1,380 & $0.35 \%$ \\
\hline Totals & 137,680 & $32.97 \%$ & 129,550 & $\mathbf{3 3 . 0 0 \%}$ \\
\hline
\end{tabular}

It would appear, although low wage jobs concentrate in a minority of occupations, the labor market is equitably balanced. With 22 major occupations, seven (31.8\%) have the bulk of low wage jobs. Table 17 explains how these seven categories account for $32.97 \%$ in 2016 and $33.00 \%$ in 2011 of all jobs, suggesting a balance in the labor market. With three typologies, however, a balanced distribution suggests low wage jobs should occupy no more than one-third 
of all positions. The problem lies in the fact that these seven occupations represent approximately $75 \%$ of all low wage jobs, meaning the labor market is not as balanced as it first appeared. Table 7 totaled the concentration of all low wage positions as $43.76 \%$ in 2011; Table 6 totaled $42.79 \%$ in 2016.

In Figure 8, changes in concentration of labor market for each of the 22 occupational categories showed nearly imperceptible differences between 2011 and 2016. The single outlier was Office and Administrative Support workers. In the five years between data groups, this occupation reduced its percentage of the labor force by $1.35 \%$. In 2011, there were an estimated 66,150 workers in Office and Administrative Support (Table 7); in 2016, only 64,720 workers held jobs in this occupation (Table 6).

Although the industry categories are not the same as OES's 22, the U. S. Census Bureau also measures long-term changes in sector populations through the Longitudinal Employer Household Dynamics (LEHD, 2017) database. Figure 9 shows extracted data for Quarter 2 in each of the years 2001, 2006, 2011, and 2016 (Appendix H). Proportionally, each industry but one appears to have increased over time by roughly the same amounts. Health Care and Social Assistance, most like the combined Healthcare Support workers and Community and Social Service workers of the OES categories, increased from 48,973 in 2001 to 63,593 in 2016 (Figure 9). Over the 15 years measured by these LEHD extracts, Health Care and Social Assistance grew by $29.9 \%(63,593 / 48,973)$. No other sector had increases remotely similar.

Trends and the variations with particular occupations aside, the most telling result of the South Dakota examination comes from a comparison of Table 8 with Table 6 for 2016 (Table 9 with Table 7 for 2011). Typology (Table 8) indicates only one occupation had a median income below the FESS threshold for a single earner. This occupation, Food Preparation and Serving, accounted for $9.92 \%$ of all workers. Table 6 calculated low wage workers, occupation by occupation, as comprising $42.79 \%$ of the total labor market. This difference indicates the remaining 32.87\% comes from Second Tier, or even Higher Wage, typologies. As Table 14 and Table 17 summarized, the highest concentration of low wage earners was in seven categories - all of which appear in Low Wage and Second Tier typologies. Precarity for earners with low incomes does not necessarily stem from unduly low hourly wages but appears to result from insufficient hours to earn full-time pay. Looking at South Dakota's labor market in detail offered a better perspective as to the types of wages workers earn.

With a relatively low cost of living, compared to major metropolitan areas like Chicago or Minneapolis, first impressions would lead the casual observer to believe the income workers bring home meets the needs of most. Using the WA method of evaluating a FESS standard for the state, then applying that value to IPUMS data for two 5-year data sets, revealed $41.24 \%$ of all workers in 2016 did not earn their half of the household's budgetary needs for a family of four (Appendix G). Although high, the number of workers with income below the FESS threshold improved by $0.50 \%$ since 2011 (Figure 13).

DOI: 10.17605/OSF.IO/QF4HY 
Most disturbingly, $77.32 \%$ of all workers in 2016 were unable to be the sole provider for the family (Appendix G). Again, this figure represents a 1.23\% improvement over 2011 (Appendix G). What makes this fact disturbing is the realistic possibility of any number of life events that could impact the earning capacity of one working adult, which would shift the burden to the remaining earner. As difficult as such a scenario may be to weather in South Dakota, is it better, worse, or like other states nationally?

\section{National Comparison}

Each of the remaining 37 labor markets studied by Pearce and Brooks (http://selfsufficiencystandard.org) were evaluated for an FESS threshold for both 2011 and 2016. Extractions from IPUMS for 2006-2010 and 2011-2015 were limited to line items with EMPSTAT $=1$ (employed) before importing to Excel. All entries with military occupational codes were deleted, then the data was organized by earned income in ascending order. Creation of a frequency column enabled population counts. Thresholds were measured at each state's unique single earner (annual) income level, and at the household annual needs level. Totaling each state's labor force allowed percentage values to be calculated for both thresholds (Appendix $\mathrm{G})$.

Figure 13 illustrates the change in proportions of each state's labor force with single earners below the FESS annual threshold. Although most states improved, there were some radically declining economies. Appendix G details the exact values used for Figure 13, which shows changes from 2011 to 2016 ranged from a $2.21 \%$ improvement to an $18.69 \%$ decline. The areas most affected by inflated costs of living and stagnated wages were Connecticut (-18.69\%), North Carolina (-11.78\%), and Virginia (-9.80\%). Although not as dramatic, Nevada's labor market also saw $7.20 \%$ more of its labor force fall below the single earner annual income threshold (Appendix G).

For earners capable of being the household's sole provider, Louisiana had the highest proportion (Appendix G). Figure 14 shows the improvement, or lack thereof, in state labor markets capable of meeting the household's needs with one income. In 2016, 67.81\% of the labor force in Louisiana could not handle the household's costs alone. All other states had higher percentages, with Connecticut topping the list at $90.90 \%$ of its work force unable to be the household's sole source of income (Appendix G).

As an interesting side note, knowing how much of the national labor market could support a household offers a nostalgic comparison for the reader. This study primarily focuses on the impact of job quality on the individual worker. Therefore, all 38 markets were ranked from highest proportion of labor force under the FESS single earner threshold to lowest (Table 12).

With the economic recovery gaining momentum in 2010, investigating the two data sets offers a good picture as to what that recovery has yielded for the frontline worker (Ruggles et al., 2015). In 2011, California had the highest proportion of its labor force under the FESS threshold; by 2016, it had improved only to the second position on the list. Table 18 indicates 
the net change made by each state in Table 12. FESS values from Table 13 show the concurrent changes in cost of living, as the proportion of workers making less than FESS changed. According to the U. S. Inflation Calculator (http://www.usinflationcalculator.com), inflation increased $6.7 \%$ from 2011 to 2016.

What Table 18 readily conveys is that the national average increase in inflation was not felt equally among states. Of the 38 markets studied, only six experienced inflation rates below 6.7\%: California (2.69\%), Louisiana (5.38\%), Pennsylvania (5.50\%), Utah (6.33\%), Texas $(6.39 \%)$, and Kentucky (6.55\%). Pennsylvania improved its proportion of low wage workers so much, it dropped 14 places in the ranking. In fact, Pennsylvania had the third greatest change among all labor markets. Unfortunately, Kentucky increased its proportion of low wage workers and rose six places in the ranking. The other four states with below par inflation stayed very near their 2011 position, only minimally changing their proportions of low wage earners.

On the other extreme, there were nine states experiencing hyperinflation from 2011 to 2016. The most staggering were Connecticut (58.06\%), North Carolina (35.66\%), and Virginia $(30.52 \%)$. These estimations of inflation are the result of comparing the FESS WA values, which mitigate outliers for a statewide value. In other words, these changes may underestimate the actual inflation experienced in these markets (Table 18).

Although state economies have fluctuated greatly between data sets, what is clear is that South Dakota is one of the more stable ones. Even with nearly half (41.24\% in 2016) of its labor force earning annual incomes below the single earner FESS threshold, the proportion of low wage earners to total earners is one of the lowest in the country (Appendix G). Table 12 ranked South Dakota as 29th in 2011 and 34th in 2016, out of 38. Decreasing the proportion of low wage earners by $0.50 \%$ resulted in a drop of five places in the ranking. Despite having a relative inflation rate of $9.59 \%$, greater than the national average, South Dakota has maintained a low unemployment rate and marginally improved its percentage of low wage workers. 
Table 18

Ranking Changes in Highest Proportion of Low Wage Workers and Changes in Cost of Living, from 2011 to 2016

\begin{tabular}{|c|c|c|c|c|c|c|}
\hline \multirow[b]{2}{*}{ State } & \multicolumn{2}{|c|}{ RANKING } & \multirow{2}{*}{$\begin{array}{c}\text { (Neg. is good) } \\
\text { Change }\end{array}$} & \multicolumn{2}{|c|}{ FESS (WA) } & \multirow{2}{*}{$\begin{array}{l}\text { (Neg. is good) } \\
\text { Change }\end{array}$} \\
\hline & 2011 & 2016 & & 2011 & 2016 & \\
\hline Alabama & 28 & 29 & -1 & 9.83 & 10.62 & $+8.04 \%$ \\
\hline Arizona & 9 & 10 & -1 & 12.78 & 13.94 & $+9.08 \%$ \\
\hline California & 1 & 2 & -1 & 16.38 & 16.82 & $+2.69 \%$ \\
\hline Colorado & 11 & 7 & +4 & 13.74 & 15.49 & $+12.74 \%$ \\
\hline Connecticut & 10 & 1 & +9 & 15.95 & 25.21 & $+58.06 \%$ \\
\hline Delaware & 34 & 33 & +1 & 11.36 & 12.18 & $+7.22 \%$ \\
\hline Florida & 6 & 5 & +1 & 12.26 & 13.19 & $+7.59 \%$ \\
\hline Georgia & 26 & 25 & +1 & 10.81 & 11.69 & $+8.14 \%$ \\
\hline Hawaii & 13 & 16 & -3 & 13.47 & 14.51 & $+7.72 \%$ \\
\hline Illinois & 5 & 13 & -8 & 13.51 & 14.55 & $+7.70 \%$ \\
\hline Indiana & 23 & 17 & -6 & 10.84 & 12.54 & $+15.68 \%$ \\
\hline Kentucky & 15 & 21 & +6 & 11.30 & 12.04 & $+6.55 \%$ \\
\hline Louisiana & 38 & 37 & +1 & 9.30 & 9.80 & $+5.38 \%$ \\
\hline Maryland & 12 & 12 & 0 & 15.42 & 17.54 & $+13.75 \%$ \\
\hline Massachusetts & 8 & 14 & -6 & 15.93 & 17.15 & $+7.66 \%$ \\
\hline Mississippi & 37 & 35 & +2 & 9.03 & 9.64 & $+6.76 \%$ \\
\hline Missouri & 30 & 31 & +1 & 9.98 & 10.79 & $+8.12 \%$ \\
\hline Montana & 14 & 23 & +9 & 10.84 & 11.68 & $+7.75 \%$ \\
\hline Nebraska & 19 & 28 & +9 & 10.91 & 11.66 & $+6.87 \%$ \\
\hline Nevada & 33 & 22 & -11 & 11.63 & 12.52 & $+7.65 \%$ \\
\hline New Jersey & 7 & 6 & +1 & 16.30 & 17.54 & $+7.61 \%$ \\
\hline New York & 3 & 4 & -1 & 15.88 & 17.10 & $+7.68 \%$ \\
\hline North Carolina & 32 & 8 & +24 & 10.01 & 13.58 & $+35.66 \%$ \\
\hline Ohio & 18 & 19 & -1 & 11.79 & 12.93 & $+9.67 \%$ \\
\hline Oklahoma & 21 & 30 & -9 & 10.04 & 10.83 & $+7.87 \%$ \\
\hline Oregon & 17 & 11 & +6 & 11.47 & 13.36 & $+16.48 \%$ \\
\hline Pennsylvania & 4 & 18 & -14 & 13.46 & 14.20 & $+5.50 \%$ \\
\hline South Carolina & 27 & 26 & +1 & 10.21 & 11.03 & $+8.03 \%$ \\
\hline South Dakota & 29 & 34 & -5 & 9.91 & 10.86 & $+9.59 \%$ \\
\hline Tennessee & 24 & 27 & -3 & 10.25 & 11.05 & $+7.80 \%$ \\
\hline Texas & 22 & 24 & -2 & 10.48 & 11.15 & $+6.39 \%$ \\
\hline Utah & 2 & 3 & -1 & 13.43 & 14.28 & $+6.33 \%$ \\
\hline Virginia & 25 & 9 & +16 & 12.32 & 16.08 & $+\mathbf{3 0 . 5 2 \%}$ \\
\hline Washington & 16 & 15 & +1 & 13.36 & 14.99 & $+12.20 \%$ \\
\hline Washington D. C. & 31 & 38 & -7 & 16.11 & 17.32 & $+7.51 \%$ \\
\hline West Virginia & 35 & 36 & -1 & 9.36 & 10.08 & $+7.69 \%$ \\
\hline Wisconsin & 20 & 20 & 0 & 11.73 & 13.20 & $+12.53 \%$ \\
\hline Wyoming & 36 & 32 & +4 & 10.46 & 12.01 & $+14.82 \%$ \\
\hline
\end{tabular}

Note: Placement information and change in placement taken from Table 12, where higher rank indicates greater proportion of labor force earning less than FESS single earner threshold. Hourly wages were taken from Table 13.

DOI: 10.17605/OSF.IO/QF4HY 


\section{DISCUSSION}

South Dakota does not have a large revenue stream to draw from when trying to finance employment growth and social programs. The state, to stimulate economic development, is structured in such a way that it receives no corporate income taxes, no personal income taxes, no personal property taxes, no business inventory taxes, and no inheritance taxes (SD, 2017). Even with its no tax policy, the state has failed to attract significant levels of employers offering quality jobs with full-time or Second Tier wages. By choosing to forego the types of taxes that provide revenue for most states, there are missed opportunities for infrastructure development more in line with what prospective new businesses may value. Over the 2006-2015 period reviewed, the retail, food service, and healthcare sectors were the predominant occupations that experienced job growth. Retail and Food Service industries also happen to be the lowest paid of all 22 occupations studied (Table 6).

To bridge the gap between operational need and revenue, approximately $60 \%$ of South Dakota's budget consists of federal funds (SD, 2017). If the conclusions offered by the Center on Budget and Policy Priorities are accurate, TANF block grants may be getting diverted "to fill state budget holes, and in some cases to substitute for existing state spending" (Schott et al., 2015, p. 1). Although public subsidies are available, South Dakota restricts funds so that only the poorest of the poor can qualify for most programs. Complicating matters, the costs of administering these programs routinely occupies a greater percentage of federal monies than the programs provide to the needy. Ironically, $56.48 \%$ of public sector workers are also low wage earners potentially at risk of needing subsidies (Table 4).

The state budget, therefore, is funded primarily from sales taxes on consumer transactions, including groceries and clothing. Consumer taxes directly impact all citizens, regardless of their resources. Structuring the state's funding by taxing those most in need of help only exacerbates the aid government may be asked to provide. With such limited resources, legislators must find ways to allocate funds to programs judiciously.

One program not discussed within this paper was the role of subsidized housing. The Sioux Falls Public Housing Authority currently has a four-year waiting list for applicants (personal communication, 2017). With $41.24 \%$ of South Dakota's work force earning less than $\$ 22,936$ per year, dramatically expanding income-contingent housing would enable even low wage earners to live more independently (Table 13). With $77.32 \%$ of the work force earning less than $\$ 45,873$ per year (Table 13), the lofty dream of home ownership seems out of reach for most residents. 


\section{CONCLUSION}

This study examined South Dakota's labor market by looking at individual earner income levels, age, and dispersion of jobs across the state to provide information vital for leaders to initiate supportive economic development. Although precarious jobs are commonplace for a student not yet on a career track, older workers may be working for lack of a retirement fund. The needs of older workers without resources are different, and potentially more serious, than the needs of younger coworkers.

Job growth has relied heavily on retail and service positions during the period from 2006 to 2015. The largest growing occupation has been Healthcare Practitioners, while less educationally demanding Healthcare Support positions have declined (Figure 8). For the average worker, leaving Retail or Food Service for an office job becomes more difficult each year. Between 2011 and 2016, Office and Administrative Support positions experienced the greatest decline of all occupations (Figure 8).

The working population of South Dakota is aging to the degree that nearly 8,000 more people over age 65 had jobs in 2016 over 2011 (Table 10). Although these older workers do make more wages than their younger counterparts, $29.67 \%$ of those Over 40 did not earn $\$ 22,936$ per year and $68.57 \%$ did not earn $\$ 45,873$ per year (Figure 12 ). The largest change in the labor force appeared in the 40-49 age group. The group with the assumed highest skill level and earning potential aged into the next group, without an adequate number of younger workers aging into the category to replace them. The 40-49 age group lost 5,258 workers from 2011 to 2016.

A need exists in South Dakota for the development of Second Tier, full-time jobs (Table 8), both to attract younger workers to the area and to keep skilled workers. Employers may need incentives to communicate the importance of employee retention and advancement opportunities (Brown, 2016; Netessine, 2014). Older workers are staying in the labor force, but not earning at least household level wages after a lifetime of working. It would appear these individuals are employed for lack of retirement options. Future research into the motivations of older workers could increase the information lawmakers need to enact any policy changes.

Although South Dakota's labor market composition compares favorably to 35 other states, the improvement is relative. Only having $77.32 \%$ of the labor market unable to support a household, and $41.24 \%$ making less than their half to pool resources, is still a troubling condition. Expecting workers to thrive by providing for their own needs, when the state leans heavily on the federal government, demands of those least capable of self-sufficiency that which the state neither enables nor exemplifies. 


\section{REFERENCES}

Argyres, A., Holgate, B., \& Moir, S. (2012). Low wage earners and low wage jobs in Greater Boston. University of Massachusetts Boston, Labor Resource Center. Retrieved from http://works.bepress.com/susan_moir/8/

Bernabè, S., \& Kolev, A. (2005). Jobless or working poor in the Kyrgyz Labour Market: What role for social policies? Social Policy \& Administration, 39(4), 409-430. doi: 10.1111/j.1467-9515.2005.00447.x

Boushey, H., Fremstad, S., Gragg, R., \& Waller, M. (2007). Understanding low-wage work in the United States. Center for Economic Policy and Research. Retrieved from http://research.policyarchive.org/20488.pdf

Broughton, Green, Rickard, Swift, Eichhorst, Tobsch, . . \& \& Tros. (2016). Precarious employment in Europe: Patterns, trends and policy strategies. Directorate General for Internal Policies. Policy Department A: Economic and Scientific Policy. Retrieved from http://www.europarl.europa.eu/RegData/etudes/STUD/2016/587285/IPOL_STU(2016)58 7285_EN.pdf

Brown, A. (2016). Key findings about the American workforce and the changing job market. Pew Research Center. Retrieved from http://www.pewresearch.org

Buchanan, R., \& Chaparro, R. (2008). International institutions and transnational advocacy: The case of the North American Agreement on Labor Cooperation. UCLA Journal of International Law \& Foreign Affairs, 13(1), 129-159. doi: 10.2139/ssrn.1262789

Choi, H.-L., \& Mattoni, A. (2010). The contentious field of precarious work in Italy; Political actors, strategies and coalitions. Working USA, 13(2), 213-243. doi: 10.1111/j.17434580.2010.00284.x

Department of Social Services. (DSS, 2017). TANF data and statistics. Lynne A. Valenti, Cabinet Secretary. Retrieved from https://dss.sd.gov

Eberstadt, N. (2006). The mismeasure of poverty. Policy Review, (138), 19-51. Retrieved from ABI/INFORM database.

Figueroa, M., Grabelsky, J., \& Lamare, R. (2011). Community workforce provisions in project labor agreements: A tool for building middle-class careers. Cornell University, ILR School. Retrieved from http://digitalcommons.ilr.cornell.edu/reports/64/

Forrant, R. (2002). The International Association of Machinists, Pratt \& Whitney, and the struggle for a blue-collar future in Connecticut. International Review of Social History, 47(S10), 113-136. doi: 10.1017/S0020859002000809

DOI: 10.17605/OSF.IO/QF4HY 
Gustafson, K. (2014, September 23). Retail's turnover a plus for economy but challenge for stores. CNBC.com. Retrieved from https://www.cnbc.com/2014/09/23/retail-turnoverrates-on-the-rise.html

Kalleberg, A. L. (2009). Precarious work, insecure workers: Employment relations in transition. American Sociological Review, 74(1), 1-22. doi: 10.1177/000312240907400101

Lettau, M. K., \& Buchmueller, T. C. (1999). Comparing benefit costs for full- and part-time workers. Monthly Labor Review, 122(3), 30-35. Retrieved from ProQuest database.

Longitudinal Employer-Household Dynamics (LEHD, 2017). Quarterly workforce indicators (QWI) data extraction, limited to quarter 2 in years 2001, 2006, 2011, and 2016. U. S. Census Bureau. Retrieved from https://lehd.ces.census.gov

Medicaid-help. (2017). Qualifications for Medicaid in South Dakota. Retrieved from https://medicaid-help.org

Minnesota Department of Human Services. (2017). Insurance Affordability Programs (IAPs) income and asset guidelines. Retrieved from https://edocs.dhs.state.mn.us/lfserver/Public/DHS-3461A-ENG

Netessine, S. (2014). Product knowledge drives sales leadership. Fontainebleau: INSEAD. Retrieved from ProQuest database.

Olsthoorn, M. (2014). Measuring precarious employment: A proposal for two indicators of precarious employment based on set-theory and tested with Dutch labor market-data. Social Indicators Research: An International and Interdisciplinary Journal for Quality-of-Life Measurement, 119(1), 421-441. doi:10.1007/s11205-013-0480-y

Pearce, D., \& Brooks, J. (2000). The self-sufficiency standard for South Dakota. Center for Women's Welfare, University of Washington. Retrieved from http://selfsufficiencystandard.org/south-dakota

Ruggles, S., Genadek, K., Goeken, R., Grover, J., and Sobek, M. (2015). Integrated Public Use Microdata Series: Version 6.0 [dataset]. Minneapolis: University of Minnesota. doi:10.18128/D010.V6.0

Schott, L., Pavetti, L., \& Floyd, I. (2015, October 15). How states use federal and state funds under the TANF block grant. Center on Budget and Policy Priorities. Retrieved from https://www.cbpp.org/sites/default/files/atoms/files/4-8-15tanf_0.pdf

South Dakota Department of Labor and Regulation (2016, October 19). South Dakota minimum wage goes up January 1, 2017. South Dakota Retailer's Association, press release. Retrieved from https://www.sdra.org/

DOI: 10.17605/OSF.IO/QF4HY 
South Dakota Kids Count. (2017, February 15). Child and Adult Nutrition Services, South Dakota Department of Education. Raw data retrieved from http://doe.sd.gov/cans/nslp.asp

South Dakota taxes. (SD, 2017). Governor's Office of Economic Development. Retrieved March 22, 2017 from http://www.sdreadytowork.com/Taxes

Spence, A. M., \& Hlatshwayo, S. (2011). The evolving structure of the American economy and the employment challenge. Council on Foreign Relations, Working Paper. Retrieved from http://www.cfr.org/industrial-policy/evolving-structure-american-economyemployment-challenge/p24366

Stoll, D. (2009). Which American Dream do you mean? Society, 46(5), 398-402. doi: $10.1007 / \mathrm{s} 12115-009-9245-2$

U. S. Census Bureau. (2016a). American Community Survey (ACS) data table, DP03: Selected economic characteristics, 2011-2015 South Dakota. Retrieved from https://www.census.gov/acs/www/data/data-tables-and-tools/american-factfinder/

U. S. Census Bureau. (2016b). American Community Survey (ACS) data table S2303: Work status in the past 12 months, May 2016. Retrieved from https://www.census.gov/acs/www/data/data-tables-and-tools/american-factfinder/

U. S. Department of Commerce, Bureau of the Census. (1979). Money income of households in the United States: 1979. Consumer Income, Series P-60, No. 126. Retrieved from https://www2.census.gov/prod2/popscan/p60-126.pdf

U. S. Department of Health and Human Services. (HHS, 2016). The 2016 HHS poverty guidelines. Retrieved from https://aspe.hhs.gov/computations-2016-poverty-guidelines

U. S. Department of Labor, Bureau of Labor Statistics. (BLS, 2016a). Earnings, Table 37. Median weekly earnings of full-time wage and salary workers by detailed occupation and sex. Retrieved from https://www.bls.gov/cps/cpsaat39.htm

U. S. Department of Labor, Bureau of Labor Statistics. (BLS, 2016b). Occupational Employment Statistics (OES) data tables. Multiple occupations for one geographical area, South Dakota, May 2016. Retrieved from https://data.bls.gov/oes/

U. S. Department of Labor, Bureau of Labor Statistics. (BLS, 2016c). Occupational Employment Statistics (OES) data tables. Multiple occupations for one geographical area, South Dakota, May 2011. Retrieved from https://data.bls.gov/oes/

U. S. Department of Labor, Bureau of Labor Statistics. (BLS, 2017). Unemployment rate. National unemployment rate for workers 16 years and over. Retrieved from https://data.bls.gov/

DOI: 10.17605/OSF.IO/QF4HY 


\section{APPENDIX A}

\section{Family Economic Self-Sufficiency Standard for South Dakota}

Table 2

The Self-Sufficiency Standard for Selected Family Types in

Sioux Falls/Minnehaha County, SD, 2000*

Monthly Expenses and Shares of Total Budgets

\begin{tabular}{|c|c|c|c|c|c|c|c|c|}
\hline \multirow[b]{2}{*}{ Monthly Costs } & \multicolumn{2}{|c|}{ One Adult } & \multicolumn{2}{|c|}{$\begin{array}{c}\text { One Adult, } \\
\text { One Preschooler }\end{array}$} & \multicolumn{2}{|c|}{$\begin{array}{c}\text { One Adult, } \\
\text { One Preschooler, } \\
\text { One Schoolage }\end{array}$} & \multicolumn{2}{|c|}{$\begin{array}{c}\text { Two Adults, } \\
\text { One Preschooler, } \\
\text { One Schoolage }\end{array}$} \\
\hline & Costs & $\begin{array}{l}\% \text { of } \\
\text { total }\end{array}$ & Costs & $\begin{array}{l}\% \text { of } \\
\text { total }\end{array}$ & Costs & $\begin{array}{l}\% \text { of } \\
\text { total }\end{array}$ & Costs & $\begin{array}{l}\text { \% of } \\
\text { total }\end{array}$ \\
\hline Housing & $\$ 475$ & 42 & $\$ 602$ & 30 & $\$ 602$ & 24 & $\$ 602$ & 20 \\
\hline Child Care & $\$ 0$ & 0 & $\$ 436$ & 22 & $\$ 744$ & 29 & $\$ 744$ & 25 \\
\hline Food & $\$ 164$ & 14 & $\$ 249$ & 13 & $\$ 372$ & 15 & $\$ 511$ & 17 \\
\hline Transportation & $\$ 150$ & 13 & $\$ 150$ & 8 & $\$ 150$ & 6 & $\$ 290$ & 10 \\
\hline Health Care & $\$ 85$ & 7 & $\$ 195$ & 10 & $\$ 216$ & 9 & $\$ 270$ & 9 \\
\hline Miscellaneous & $\$ 87$ & 8 & $\$ 163$ & 8 & $\$ 209$ & 8 & $\$ 242$ & 8 \\
\hline Taxes & $\$ 182$ & 16 & $\$ 320$ & 16 & $\$ 421$ & 17 & $\$ 483$ & 16 \\
\hline $\begin{array}{l}\text { Earned Income } \\
\text { Tax Credit (-) }\end{array}$ & $\$ 0$ & 0 & $\$ 49$ & -2 & $-\$ 12$ & 0 & $\$ 0$ & 0 \\
\hline $\begin{array}{l}\text { Child Care } \\
\text { Tax Credit (-) }\end{array}$ & $\$ 0$ & 0 & $-\$ 46$ & -2 & $-\$ 80$ & -3 & $-\$ 80$ & -3 \\
\hline Child Tax Credit (-) & \$o & 0 & $\$ 42$ & -2 & $-\$ 83$ & -3 & $-\$ 83$ & -3 \\
\hline $\begin{array}{l}\text { Self-Sufficiency } \\
\text { Wage - } \\
\text { Monthly } \\
\text { Hourty }\end{array}$ & $\begin{array}{r}\$ 1,143 \\
\$ C\end{array}$ & 100 & $\begin{array}{r}\$ 1,981 \\
\$ 1\end{array}$ & 100 & $\begin{array}{r}\$ 2,539 \\
\$ 1\end{array}$ & 100 & $\begin{array}{r}\$ 2,978 \\
\$ 8.46\end{array}$ & $\begin{array}{l}100 \\
\text { er adult }\end{array}$ \\
\hline
\end{tabular}

- The Standard is calculated by adding expenses and taves and subtracting tax credits.

- The houlty wage is calculated by dhiding the monthy uage by 176 hours (8 hours per day times 22 days per morth). Nole: Totals may not add exactly die to rounding.

per month) to be able to meet her/his basic needs, as can be seen in the first column of Table 1. While these costs are high, a single parent with one preschooler needs to earn about $70 \%$ more at $\mathbf{\$ 1 0 . 2 6}$ per hour (\$1,805 per month) to meet the basic needs of her family. ${ }^{18}$ If she has two children, one preschooler and one schoolage child, she would need about twice as much, $\mathbf{S 1 2 . 7 0}$ per hour (\$2,235 per month) to meet her family's needs (see Table 1). Finally, if there are two adults supporting two children, a preschooler and a school-age child, costs are increased slightly for additional food, health care, and miscellaneous costs for the second adult, but the major costs of housing and child care stay the same. Thus, the amount each would need

The Self-Sufficiency Standard for South Dakota to earn is only about three-fifths the amount a single parent would need to earn: $\mathbf{\$ 7 . 7 8}$ per hour ( $\mathbf{\$ 2 , 7 3 8}$ per month total).

In Sioux Falls/Minnehaha County (see Table 2), the costs are slightly higher than in Rapid City for each family type. Thus, a single adult's Self-Sufficiency Wage is \$6.50 per hour, and again the single parent must earn about $70 \%$ more, or $\mathbf{\$ 1 1 . 2 5}$ per hour (\$1,981 per month). The single parent with two children in Sioux Falls would need to earn $\$ 14.42$ per hour (\$2,539 per month) to meet her family's needs (see Table 2), and in the two-parent family, each adult would need to earn \$8.46 per hour or a total of \$2,978 per month.

Retrieved from http://selfsufficiencystandard.org/south-dakota 


\section{APPENDIX B \\ Table DP03: Selected economic characteristic, 2011-2015 American Community Survey 5-year Estimates}

Although the American Community Survey (ACS) produces population, demographic and housing unit estimates, it is the Census Bureau's Population Estimates Program that produces and disseminates the official estimates of the population for the nation, states, counties, cities and towns and estimates of housing units for states and counties.

\begin{tabular}{|c|c|c|c|c|}
\hline \multirow[t]{2}{*}{ Subject } & \multicolumn{4}{|c|}{ South Dakota } \\
\hline & Estimate & $\begin{array}{l}\text { Margin of } \\
\text { Error }\end{array}$ & Percent & $\begin{array}{l}\text { Percent Margin } \\
\text { of Error }\end{array}$ \\
\hline \multicolumn{5}{|l|}{ EMPLOYMENT STATUS } \\
\hline Population 16 years and over & 657,804 & $+/-510$ & 657,804 & $(\mathrm{X})$ \\
\hline In labor force & 453,990 & $+/-1,831$ & $69.0 \%$ & $+/-0.3$ \\
\hline Civilian labor force & 451,073 & $+/-1,906$ & $68.6 \%$ & $+/-0.3$ \\
\hline Employed & 430,853 & $+/-1,972$ & $65.5 \%$ & $+/-0.3$ \\
\hline Unemployed & 20,220 & $+/-844$ & $3.1 \%$ & $+/-0.1$ \\
\hline Armed Forces & 2,917 & $+/-315$ & $0.4 \%$ & $+/-0.1$ \\
\hline Not in labor force & 203,814 & $+/-1,819$ & $31.0 \%$ & $+/-0.3$ \\
\hline Civilian labor force & 451,073 & $+/-1,906$ & 451,073 & $(\mathrm{X})$ \\
\hline Unemployment Rate & $(\mathrm{X})$ & $(\mathrm{X})$ & $4.5 \%$ & $+/-0.2$ \\
\hline Females 16 years and over & 329,772 & $+/-527$ & 329,772 & $(\mathrm{X})$ \\
\hline In labor force & 213,403 & $+/-1,348$ & $64.7 \%$ & $+/-0.4$ \\
\hline Civilian labor force & 212,989 & $+/-1,368$ & $64.6 \%$ & $+/-0.4$ \\
\hline Employed & 204,179 & $+/-1,382$ & $61.9 \%$ & $+/-0.4$ \\
\hline $\begin{array}{l}\text { Own children of the householder under } 6 \\
\text { years }\end{array}$ & 69,049 & $+/-620$ & 69,049 & $(\mathrm{X})$ \\
\hline All parents in family in labor force & 51,671 & $+/-1,053$ & $74.8 \%$ & $+/-1.4$ \\
\hline $\begin{array}{l}\text { Own children of the householder } 6 \text { to } 17 \\
\text { years }\end{array}$ & 126,995 & $+/-702$ & 126,995 & $(\mathrm{X})$ \\
\hline All parents in family in labor force & 103,448 & $+/-1,523$ & $81.5 \%$ & $+/-1.1$ \\
\hline \multicolumn{5}{|l|}{ COMMUTING TO WORK } \\
\hline Workers 16 years and over & 425,712 & $\begin{array}{r}+/- \\
1,975\end{array}$ & 425,712 & $(\mathrm{X})$ \\
\hline Car, truck, or van -- drove alone & 337,032 & $+/-1,943$ & $79.2 \%$ & $+/-0.3$ \\
\hline Car, truck, or van -- carpooled & 39,586 & $+/-1,373$ & $9.3 \%$ & $+/-0.3$ \\
\hline Public transportation (excluding taxicab) & 2,242 & $+/-353$ & $0.5 \%$ & $+/-0.1$ \\
\hline Walked & 16,811 & $+/-798$ & $3.9 \%$ & $+/-0.2$ \\
\hline Other means & 6,342 & $+/-621$ & $1.5 \%$ & $+/-0.1$ \\
\hline Worked at home & 23,699 & $+/-884$ & $5.6 \%$ & $+/-0.2$ \\
\hline Mean travel time to work (minutes) & 16.9 & $+/-0.2$ & $(\mathrm{X})$ & $(\mathrm{X})$ \\
\hline
\end{tabular}

DOI: 10.17605/OSF.IO/QF4HY 


\section{APPENDIX B}

Table DP03: Selected economic characteristic, 2011-2015 American Community Survey 5-year Estimates (continued)

\begin{tabular}{|c|c|c|c|c|}
\hline \multirow[t]{2}{*}{ Subject } & \multicolumn{4}{|c|}{ South Dakota } \\
\hline & Estimate & $\begin{array}{l}\text { Margin of } \\
\text { Error }\end{array}$ & Percent & $\begin{array}{l}\text { Percent Margin } \\
\text { of Error }\end{array}$ \\
\hline \multicolumn{5}{|l|}{ OCCUPATION } \\
\hline $\begin{array}{l}\text { Civilian employed population } 16 \text { years and } \\
\text { over }\end{array}$ & 430,853 & $\begin{array}{r}+/- \\
1,972 \\
\end{array}$ & 430,853 & $(\mathrm{X})$ \\
\hline $\begin{array}{r}\text { Management, business, science, and arts } \\
\text { occupations }\end{array}$ & 150,143 & $+/-2,047$ & $34.8 \%$ & $+/-0.4$ \\
\hline Service occupations & 74,626 & $+/-1,521$ & $17.3 \%$ & $+/-0.3$ \\
\hline Sales and office occupations & 103,270 & $+/-1,890$ & $24.0 \%$ & $+/-0.4$ \\
\hline $\begin{array}{l}\text { Natural resources, construction, and } \\
\text { maintenance occupations }\end{array}$ & 47,344 & $+/-1,167$ & $11.0 \%$ & $+/-0.3$ \\
\hline $\begin{array}{l}\text { Production, transportation, and material } \\
\text { moving occupations }\end{array}$ & 55,470 & $+/-1,579$ & $12.9 \%$ & $+/-0.4$ \\
\hline \multicolumn{5}{|l|}{ INDUSTRY } \\
\hline $\begin{array}{l}\text { Civilian employed population } 16 \text { years and } \\
\text { over }\end{array}$ & 430,853 & $\begin{array}{r}+/- \\
1,972 \\
\end{array}$ & 430,853 & $(\mathrm{X})$ \\
\hline $\begin{array}{r}\text { Agriculture, forestry, fishing and hunting, and } \\
\text { mining }\end{array}$ & 30,229 & $+/-767$ & $7.0 \%$ & $+/-0.2$ \\
\hline Construction & 29,258 & $+/-1,067$ & $6.8 \%$ & $+/-0.3$ \\
\hline Manufacturing & 42,605 & $+/-1,326$ & $9.9 \%$ & $+/-0.3$ \\
\hline Wholesale trade & 12,771 & $+/-686$ & $3.0 \%$ & $+/-0.2$ \\
\hline Retail trade & 48,421 & $+/-1,406$ & $11.2 \%$ & $+/-0.3$ \\
\hline Transportation and warehousing, and utilities & 17,945 & $+/-778$ & $4.2 \%$ & $+/-0.2$ \\
\hline Information & 7,482 & $+/-464$ & $1.7 \%$ & $+/-0.1$ \\
\hline $\begin{array}{l}\text { Finance and insurance, and real estate and } \\
\text { rental and leasing }\end{array}$ & 31,183 & $+/-1,239$ & $7.2 \%$ & $+/-0.3$ \\
\hline $\begin{array}{l}\text { Professional, scientific, and management, and } \\
\text { administrative and waste management services }\end{array}$ & 25,751 & $+/-1,066$ & $6.0 \%$ & $+/-0.2$ \\
\hline $\begin{array}{l}\text { Educational services, and health care and } \\
\text { social assistance }\end{array}$ & 103,714 & $+/-2,018$ & $24.1 \%$ & $+/-0.5$ \\
\hline $\begin{array}{l}\text { Arts, entertainment, and recreation, and } \\
\text { accommodation and food services }\end{array}$ & 40,350 & $+/-1,502$ & $9.4 \%$ & $+/-0.4$ \\
\hline Other services, except public administration & 19,450 & $+/-848$ & $4.5 \%$ & $+/-0.2$ \\
\hline Public administration & 21,694 & $+/-978$ & $5.0 \%$ & $+/-0.2$ \\
\hline \multicolumn{5}{|l|}{ CLASS OF WORKER } \\
\hline $\begin{array}{l}\text { Civilian employed population } 16 \text { years and } \\
\text { over }\end{array}$ & 430,853 & $\begin{array}{r}+/- \\
1,972\end{array}$ & 430,853 & $(\mathrm{X})$ \\
\hline Private wage and salary workers & 327,500 & $+/-2,140$ & $76.0 \%$ & $+/-0.4$ \\
\hline Government workers & 66,678 & $+/-1,609$ & $15.5 \%$ & $+/-0.4$ \\
\hline $\begin{array}{l}\text { Self-employed in own not incorporated } \\
\text { business workers }\end{array}$ & 35,515 & $+/-1,072$ & $8.2 \%$ & $+/-0.2$ \\
\hline Unpaid family workers & 1,160 & $+/-148$ & $0.3 \%$ & $+/-0.1$ \\
\hline
\end{tabular}

(continued)

DOI: 10.17605/OSF.IO/QF4HY 


\section{APPENDIX B}

Table DP03: Selected economic characteristic, 2011-2015 American Community Survey 5-year Estimates (continued)

\begin{tabular}{|c|c|c|c|c|}
\hline \multirow[t]{2}{*}{ Subject } & \multicolumn{4}{|c|}{ South Dakota } \\
\hline & Estimate & $\begin{array}{l}\text { Margin of } \\
\text { Error }\end{array}$ & Percent & $\begin{array}{l}\text { Percent Margin } \\
\text { of Error }\end{array}$ \\
\hline $\begin{array}{l}\text { INCOME AND BENEFITS (IN } 2015 \\
\text { INFLATION-ADJUSTED DOLLARS) }\end{array}$ & & & & \\
\hline Total households & 330,858 & $\begin{array}{r}+/- \\
1,744\end{array}$ & 330,858 & $(\mathrm{X})$ \\
\hline Less than $\$ 10,000$ & 20,406 & $+/-1,027$ & $6.2 \%$ & $+/-0.3$ \\
\hline$\$ 10,000$ to $\$ 14,999$ & 18,403 & $+/-793$ & $5.6 \%$ & $+/-0.2$ \\
\hline$\$ 15,000$ to $\$ 24,999$ & 35,437 & $+/-1,117$ & $10.7 \%$ & $+/-0.3$ \\
\hline$\$ 25,000$ to $\$ 34,999$ & 36,985 & $+/-1,241$ & $11.2 \%$ & $+/-0.4$ \\
\hline$\$ 35,000$ to $\$ 49,999$ & 50,925 & $+/-1,405$ & $15.4 \%$ & $+/-0.4$ \\
\hline$\$ 50,000$ to $\$ 74,999$ & 65,458 & $+/-1,393$ & $19.8 \%$ & $+/-0.4$ \\
\hline$\$ 75,000$ to $\$ 99,999$ & 45,058 & $+/-1,129$ & $13.6 \%$ & $+/-0.3$ \\
\hline$\$ 100,000$ to $\$ 149,999$ & 37,407 & $+/-1,043$ & $11.3 \%$ & $+/-0.3$ \\
\hline$\$ 150,000$ to $\$ 199,999$ & 10,046 & $+/-610$ & $3.0 \%$ & $+/-0.2$ \\
\hline$\$ 200,000$ or more & 10,733 & $+/-621$ & $3.2 \%$ & $+/-0.2$ \\
\hline Median household income (dollars) & 50,957 & $+/-461$ & $(\mathrm{X})$ & $(\mathrm{X})$ \\
\hline Mean household income (dollars) & 66,404 & $+/-643$ & $(\mathrm{X})$ & $(\mathrm{X})$ \\
\hline With earnings & 268,756 & $+/-1,944$ & $81.2 \%$ & $+/-0.3$ \\
\hline Mean earnings (dollars) & 65,169 & $+/-695$ & $(\mathrm{X})$ & $(\mathrm{X})$ \\
\hline With Social Security & 96,450 & $+/-1,102$ & $29.2 \%$ & $+/-0.3$ \\
\hline Mean Social Security income (dollars) & 16,593 & $+/-166$ & $(\mathrm{X})$ & $(\mathrm{X})$ \\
\hline With retirement income & 49,096 & $+/-1,007$ & $14.8 \%$ & $+/-0.3$ \\
\hline Mean retirement income (dollars) & 20,870 & $+/-661$ & $(\mathrm{X})$ & (X) \\
\hline With Supplemental Security Income & 13,178 & $+/-691$ & $4.0 \%$ & $+/-0.2$ \\
\hline Mean Supplemental Security Income (dollars) & 8,976 & $+/-327$ & $(\mathrm{X})$ & $(\mathrm{X})$ \\
\hline With cash public assistance income & 8,897 & $+/-543$ & $2.7 \%$ & $+/-0.2$ \\
\hline Mean cash public assistance income (dollars) & 2,884 & $+/-244$ & $(\mathrm{X})$ & $(\mathrm{X})$ \\
\hline $\begin{array}{l}\text { With Food Stamp/SNAP benefits in the past } \\
12 \text { months }\end{array}$ & 36,865 & $+/-1,007$ & $11.1 \%$ & $+/-0.3$ \\
\hline FAMILIES & 212,994 & $+/-1,763$ & 212,994 & $(\mathrm{X})$ \\
\hline Less than $\$ 10,000$ & 7,646 & $+/-510$ & $3.6 \%$ & $+/-0.2$ \\
\hline$\$ 10,000$ to $\$ 14,999$ & 5,617 & $+/-441$ & $2.6 \%$ & $+/-0.2$ \\
\hline$\$ 15,000$ to $\$ 24,999$ & 15,077 & $+/-675$ & $7.1 \%$ & $+/-0.3$ \\
\hline$\$ 25,000$ to $\$ 34,999$ & 18,781 & $+/-803$ & $8.8 \%$ & $+/-0.4$ \\
\hline$\$ 35,000$ to $\$ 49,999$ & 30,362 & $+/-1,171$ & $14.3 \%$ & $+/-0.5$ \\
\hline$\$ 50,000$ to $\$ 74,999$ & 46,843 & $+/-1,144$ & $22.0 \%$ & $+/-0.5$ \\
\hline$\$ 75,000$ to $\$ 99,999$ & 37,132 & $+/-1,089$ & $17.4 \%$ & $+/-0.5$ \\
\hline
\end{tabular}

DOI: $10.17605 / O S F . I O / Q F 4 H Y$ 


\section{APPENDIX B}

Table DP03: Selected economic characteristic, 2011-2015 American Community Survey 5-year Estimates (continued)

\begin{tabular}{|c|c|c|c|c|}
\hline \multirow[t]{2}{*}{ Subject } & \multicolumn{4}{|c|}{ South Dakota } \\
\hline & Estimate & $\begin{array}{l}\text { Margin of } \\
\text { Error }\end{array}$ & Percent & $\begin{array}{l}\text { Percent Margin } \\
\text { of Error }\end{array}$ \\
\hline$\$ 100,000$ to $\$ 149,999$ & 33,067 & $+/-983$ & $15.5 \%$ & $+/-0.4$ \\
\hline$\$ 150,000$ to $\$ 199,999$ & 8,922 & $+/-546$ & $4.2 \%$ & $+/-0.2$ \\
\hline$\$ 200,000$ or more & 9,547 & $+/-593$ & $4.5 \%$ & $+/-0.3$ \\
\hline Median family income (dollars) & 65,237 & $+/-655$ & $(\mathrm{X})$ & $(\mathrm{X})$ \\
\hline Mean family income (dollars) & 80,037 & $+/-969$ & $(\mathrm{X})$ & $(\mathrm{X})$ \\
\hline Per capita income (dollars) & 26,747 & $+/-231$ & $(\mathrm{X})$ & (X) \\
\hline Nonfamily households & 117,864 & $+/-1,709$ & 117,864 & (X) \\
\hline Median nonfamily income (dollars) & 30,316 & $+/-424$ & $(\mathrm{X})$ & (X) \\
\hline Mean nonfamily income (dollars) & 39,198 & $+/-614$ & $(\mathrm{X})$ & $(\mathrm{X})$ \\
\hline Median earnings for workers (dollars) & 28,415 & $+/-326$ & $(\mathrm{X})$ & $(\mathrm{X})$ \\
\hline $\begin{array}{l}\text { Median earnings for male full-time, year- } \\
\text { round workers (dollars) }\end{array}$ & 41,990 & $+/-271$ & $(\mathrm{X})$ & (X) \\
\hline $\begin{array}{l}\text { Median earnings for female full-time, year- } \\
\text { round workers (dollars) }\end{array}$ & 32,302 & $+/-239$ & $(\mathrm{X})$ & $(\mathrm{X})$ \\
\hline HEALTH INSURANCE COVERAGE & & & & \\
\hline Civilian noninstitutionalized population & 825,506 & $+/-312$ & 825,506 & $(\mathrm{X})$ \\
\hline With health insurance coverage & 734,488 & $+/-2,287$ & $89.0 \%$ & $+/-0.3$ \\
\hline With private health insurance & 596,647 & $+/-3,520$ & $72.3 \%$ & $+/-0.4$ \\
\hline With public coverage & 244,046 & $+/-2,927$ & $29.6 \%$ & $+/-0.4$ \\
\hline No health insurance coverage & 91,018 & $+/-2,383$ & $11.0 \%$ & $+/-0.3$ \\
\hline $\begin{array}{l}\text { Civilian noninstitutionalized population } 18 \text { to } \\
64 \text { years }\end{array}$ & 500,083 & $+/-640$ & 500,083 & $(\mathrm{X})$ \\
\hline In labor force: & 414,301 & $+/-1,798$ & 414,301 & $(\mathrm{X})$ \\
\hline Employed: & 395,854 & $+/-1,870$ & 395,854 & $(\mathrm{X})$ \\
\hline With health insurance coverage & 344,563 & $+/-2,396$ & $87.0 \%$ & $+/-0.4$ \\
\hline With private health insurance & 330,605 & $+/-2,488$ & $83.5 \%$ & $+/-0.4$ \\
\hline With public coverage & 25,902 & $+/-1,019$ & $6.5 \%$ & $+/-0.3$ \\
\hline No health insurance coverage & 51,291 & $+/-1,698$ & $13.0 \%$ & $+/-0.4$ \\
\hline Unemployed: & 18,447 & $+/-797$ & 18,447 & $(\mathrm{X})$ \\
\hline With health insurance coverage & 9,018 & $+/-592$ & $48.9 \%$ & $+/-2.3$ \\
\hline With private health insurance & 5,133 & $+/-407$ & $27.8 \%$ & $+/-1.9$ \\
\hline With public coverage & 4,314 & $+/-386$ & $23.4 \%$ & $+/-1.9$ \\
\hline No health insurance coverage & 9,429 & $+/-574$ & $51.1 \%$ & $+/-2.3$ \\
\hline Not in labor force: & 85,782 & $+/-1,539$ & 85,782 & (X) \\
\hline With health insurance coverage & 68,773 & $+/-1,478$ & $80.2 \%$ & $+/-1.0$ \\
\hline With private health insurance & 48,089 & $+/-1,379$ & $56.1 \%$ & $+/-1.2$ \\
\hline With public coverage & 27,890 & $+/-1,077$ & $32.5 \%$ & $+/-1.2$ \\
\hline No health insurance coverage & 17,009 & $+/-887$ & $19.8 \%$ & $+/-1.0$ \\
\hline
\end{tabular}

(continued)

DOI: 10.17605/OSF.IO/QF4HY 


\section{APPENDIX B}

Table DP03: Selected economic characteristic, 2011-2015 American Community Survey 5-year Estimates (continued)

\begin{tabular}{|c|c|c|c|c|}
\hline \multirow{2}{*}{ Subject } & \multicolumn{4}{|c|}{ South Dakota } \\
\hline & Estimate & $\begin{array}{l}\text { Margin of } \\
\text { Error }\end{array}$ & Percent & $\begin{array}{l}\text { Percent Margin } \\
\text { of Error }\end{array}$ \\
\hline $\begin{array}{l}\text { PERCENTAGE OF FAMILIES AND } \\
\text { PEOPLE WHOSE INCOME IN THE PAST } \\
12 \text { MONTHS IS BELOW THE POVERTY } \\
\text { LEVEL }\end{array}$ & & & & \\
\hline All families & $(\mathrm{X})$ & $(\mathrm{X})$ & $9.1 \%$ & $+/-0.4$ \\
\hline $\begin{array}{l}\text { With related children of the householder under } \\
18 \text { years }\end{array}$ & $(\mathrm{X})$ & $(\mathrm{X})$ & $15.3 \%$ & $+/-0.7$ \\
\hline $\begin{array}{r}\text { With related children of the householder under } \\
5 \text { years only }\end{array}$ & $(\mathrm{X})$ & $(\mathrm{X})$ & $18.0 \%$ & $+/-1.6$ \\
\hline Married couple families & $(\mathrm{X})$ & $(\mathrm{X})$ & $3.9 \%$ & $+/-0.2$ \\
\hline $\begin{array}{r}\text { With related children of the householder under } \\
18 \text { years }\end{array}$ & $(\mathrm{X})$ & $(\mathrm{X})$ & $5.9 \%$ & $+/-0.5$ \\
\hline $\begin{array}{r}\text { With related children of the householder under } \\
5 \text { years only }\end{array}$ & $(\mathrm{X})$ & $(\mathrm{X})$ & $4.7 \%$ & $+/-1.0$ \\
\hline $\begin{array}{r}\text { Families with female householder, no husband } \\
\text { present }\end{array}$ & $(\mathrm{X})$ & $(\mathrm{X})$ & $32.2 \%$ & $+/-1.8$ \\
\hline $\begin{array}{r}\text { With related children of the householder under } \\
18 \text { years }\end{array}$ & $(\mathrm{X})$ & $(\mathrm{X})$ & $37.7 \%$ & $+/-2.1$ \\
\hline $\begin{array}{r}\text { With related children of the householder under } \\
5 \text { years only }\end{array}$ & $(\mathrm{X})$ & $(\mathrm{X})$ & $46.9 \%$ & $+/-4.7$ \\
\hline All people & $(\mathrm{X})$ & $(\mathrm{X})$ & $14.1 \%$ & $+/-0.4$ \\
\hline Under 18 years & $(\mathrm{X})$ & $(\mathrm{X})$ & $18.6 \%$ & $+/-0.8$ \\
\hline $\begin{array}{r}\text { Related children of the householder } \\
\text { under } 18 \text { years }\end{array}$ & $(\mathrm{X})$ & $(\mathrm{X})$ & $18.2 \%$ & $+/-0.8$ \\
\hline $\begin{array}{r}\text { Related children of the householder under } 5 \\
\text { years }\end{array}$ & $(\mathrm{X})$ & $(\mathrm{X})$ & $23.3 \%$ & $+/-1.3$ \\
\hline $\begin{array}{r}\text { Related children of the householder } 5 \text { to } 17 \\
\text { years }\end{array}$ & (X) & $(\mathrm{X})$ & $16.1 \%$ & $+/-0.8$ \\
\hline 18 years and over & $(\mathrm{X})$ & $(\mathrm{X})$ & $12.6 \%$ & $+/-0.3$ \\
\hline 18 to 64 years & $(\mathrm{X})$ & $(\mathrm{X})$ & $13.2 \%$ & $+/-0.3$ \\
\hline 65 years and over & (X) & $(\mathrm{X})$ & $10.1 \%$ & $+/-0.5$ \\
\hline People in families & $(\mathrm{X})$ & $(\mathrm{X})$ & $11.0 \%$ & $+/-0.4$ \\
\hline Unrelated individuals 15 years and over & $(\mathrm{X})$ & $(\mathrm{X})$ & $26.1 \%$ & $+/-0.7$ \\
\hline
\end{tabular}

Source: U.S. Census Bureau, 2011-2015 American Community Survey 5-Year Estimates

Explanation of Symbols:

1. An '**' entry in the margin of error column indicates that either no sample observations or too few sample observations were available to compute a standard error and thus the margin of error. A statistical test is not appropriate.

2. An '-' entry in the estimate column indicates that either no sample observations or too few sample observations were available to compute an estimate, or a ratio of medians cannot be calculated because one or both median estimates falls in the lowest interval or upper interval of an open-ended distribution.

3. An '-' following a median estimate means the median falls in the lowest interval of an open-ended distribution.

4. An ' + ' following a median estimate means the median falls in the upper interval of an open-ended distribution.

5. An '***' entry in the margin of error column indicates that the median falls in the lowest interval or upper interval of an open-ended distribution. A statistical test is not appropriate.

6. An '*****' entry in the margin of error column indicates that the estimate is controlled. A statistical test for sampling variability is not appropriate.

7. An ' $\mathrm{N}$ ' entry in the estimate and margin of error columns indicates that data for this geographic area cannot be displayed because the number of sample cases is too small.

8. An '(X)' means that the estimate is not applicable or not available.

Retrieved from https:/www.census.gov/acs/www/data/data-tables-and-tools/american-factfinder/ 
APPENDIX C

\section{Table S2303: Work status in the past 12 months for South Dakota, 2011-2015 ACS 5-year Estimates, May 2016}

\begin{tabular}{|c|c|c|c|c|c|c|c|c|c|c|c|c|}
\hline \multirow[b]{3}{*}{ Subject } & \multicolumn{12}{|c|}{ South Dakota } \\
\hline & \multicolumn{2}{|c|}{ Total } & \multicolumn{2}{|c|}{ Percent Total } & \multicolumn{2}{|c|}{ Male } & \multicolumn{2}{|c|}{ Percent Male } & \multicolumn{2}{|c|}{ Female } & \multicolumn{2}{|c|}{ Percent Female } \\
\hline & Estimate & $\begin{array}{l}\text { Margin } \\
\text { of Error }\end{array}$ & Estimate & $\begin{array}{l}\text { Margin } \\
\text { of Error }\end{array}$ & Estimate & $\begin{array}{l}\text { Margin } \\
\text { of Error }\end{array}$ & Estimate & $\begin{array}{l}\text { Margin } \\
\text { of Error }\end{array}$ & Estimate & $\begin{array}{l}\text { Margin } \\
\text { of Error }\end{array}$ & Estimate & $\begin{array}{c}\text { Margin } \\
\text { of } \\
\text { Error }\end{array}$ \\
\hline Population 16 to 64 years & 532,191 & $+1-589$ & 532,191 & $+/-589$ & 271,535 & $+/-533$ & 271,535 & $+/-533$ & 260,656 & $+/-565$ & 260,656 & $+/-565$ \\
\hline \multicolumn{13}{|l|}{ WEEKS WORKED } \\
\hline Worked 50 to 52 weeks & 344,606 & $+/-2,083$ & $64.8 \%$ & $+/-0.4$ & 185,678 & $+1-1,450$ & $68.4 \%$ & $+1-0.5$ & 158,928 & $+/-1,352$ & $61.0 \%$ & $+/-0.5$ \\
\hline Worked 48 to 49 weeks & 8,335 & $+1-538$ & $1.6 \%$ & ++-0.1 & 4,187 & $+1-402$ & $1.5 \%$ & $+1-0.1$ & 4,148 & $+1-402$ & $1.6 \%$ & $+1-0.2$ \\
\hline Worked 40 to 47 weeks & 21,609 & $+1-943$ & $4.1 \%$ & $+1-0.2$ & 9,640 & $+1-639$ & $3.6 \%$ & $+1-0.2$ & 11,969 & $+/-610$ & $4.6 \%$ & $+1-0.2$ \\
\hline Worked 27 to 39 weeks & 25,789 & $+1-919$ & $4.8 \%$ & $+1-0.2$ & 11,802 & $+/-643$ & $4.3 \%$ & $+1-0.2$ & 13,987 & $+1-725$ & $5.4 \%$ & $+1-0.3$ \\
\hline Worked 14 to 26 weeks & 21,839 & $+/-904$ & $4.1 \%$ & $+1-0.2$ & 11,129 & $+/-704$ & $4.1 \%$ & $+/-0.3$ & 10,710 & $+/-678$ & $4.1 \%$ & $+1-0.3$ \\
\hline Worked 1 to 13 weeks & 29,016 & $+1-917$ & $5.5 \%$ & $+1-0.2$ & 15,078 & $+1-676$ & $5.6 \%$ & $+1-0.2$ & 13,938 & $+1-763$ & $5.3 \%$ & $+1-0.3$ \\
\hline Did not work & 80,997 & $+/-1,551$ & $15.2 \%$ & $+1-0.3$ & 34,021 & $+/-1,140$ & $12.5 \%$ & $+1-0.4$ & 46,976 & $+/-998$ & $18.0 \%$ & $+1-0.4$ \\
\hline \multicolumn{13}{|l|}{ USUAL HOURS WORKED } \\
\hline $\begin{array}{l}\text { Usually worked } 35 \text { or more } \\
\text { hours per week }\end{array}$ & 352,842 & $+1-2,078$ & $66.3 \%$ & $+1-0.4$ & 201,318 & $+/-1,507$ & $74.1 \%$ & $+1-0.6$ & 151,524 & $+/-1,409$ & $58.1 \%$ & $+/ .0 .5$ \\
\hline 50 to 52 weeks & 298,888 & $+/-2,098$ & $56.2 \%$ & $+1-0.4$ & 170,758 & $+/-1,407$ & $62.9 \%$ & $+1-0.5$ & 128,130 & $+/-1,586$ & $49.2 \%$ & $+1-0.6$ \\
\hline 48 to 49 weeks & 5,328 & $+1-455$ & $1.0 \%$ & $+/-0.1$ & 3,143 & $+/-364$ & $1.2 \%$ & $+/-0.1$ & 2,185 & $+1-254$ & $0.8 \%$ & $+/-0.1$ \\
\hline 40 to 47 weeks & 13,384 & $+1-706$ & $2.5 \%$ & $+/-0.1$ & 6,807 & $+/ .484$ & $2.5 \%$ & $+1-0.2$ & 6,577 & +1.463 & $2.5 \%$ & $+1-0.2$ \\
\hline 27 to 39 weeks & 13,488 & $+1-684$ & $2.5 \%$ & $+1-0.1$ & 7,152 & $+1-573$ & $2.6 \%$ & $+1-0.2$ & 6,336 & $+1-508$ & $2.4 \%$ & $+1-0.2$ \\
\hline 14 to 26 weeks & 10,883 & $+1-722$ & $2.0 \%$ & $+/-0.1$ & 6,810 & $+1-653$ & $2.5 \%$ & $+1-0.2$ & 4,073 & $+1-435$ & $1.6 \%$ & $+1-0.2$ \\
\hline 1 to 13 weeks & 10,871 & $+/-618$ & $2.0 \%$ & $+1-0.1$ & 6,648 & $+1-503$ & $2.4 \%$ & $+1-0.2$ & 4,223 & $+1-364$ & $1.6 \%$ & $+1-0.1$ \\
\hline $\begin{array}{l}\text { Usually worked } 15 \text { to } 34 \\
\text { hours per week }\end{array}$ & 76,213 & $+1-1,836$ & $14.3 \%$ & $+1-0.3$ & 27,477 & $+1-1,077$ & $10.1 \%$ & $+1-0.4$ & 48,736 & $+1-1,242$ & $18.7 \%$ & $+1-0.5$ \\
\hline 50 to 52 weeks & 38,725 & $+/-1,245$ & $7.3 \%$ & $+1-0.2$ & 12,280 & $+/-769$ & $4.5 \%$ & $+1-0.3$ & 26,445 & $+1-844$ & $10.1 \%$ & $+1-0.3$ \\
\hline 48 to 49 weeks & 2,506 & $+/-358$ & $0.5 \%$ & $+/-0.1$ & 914 & $+/-201$ & $0.3 \%$ & $+/-0.1$ & 1,592 & $+1-291$ & $0.6 \%$ & $+/-0.1$ \\
\hline 40 to 47 weeks & 6,490 & $+1-496$ & $1.2 \%$ & $+1-0.1$ & 2,297 & $+1-318$ & $0.8 \%$ & $+/-0.1$ & 4,193 & $+1-395$ & $1.6 \%$ & $+1-0.2$ \\
\hline 27 to 39 weeks & 9,344 & $+/-593$ & $1.8 \%$ & ++-0.1 & 3,668 & $+1-377$ & $1.4 \%$ & $+/-0.1$ & 5,676 & $+/-477$ & $2.2 \%$ & $+1-0.2$ \\
\hline 14 to 26 weeks & 7,808 & $+1-666$ & $1.5 \%$ & $+1-0.1$ & 3,078 & $+1-346$ & $1.1 \%$ & $+1-0.1$ & 4,730 & $+1-504$ & $1.8 \%$ & $+1-0.2$ \\
\hline 1 to 13 weeks & 11,340 & $+1-739$ & $2.1 \%$ & $+1-0.1$ & 5,240 & $+1-421$ & $1.9 \%$ & $+1-0.2$ & 6,100 & $+1-528$ & $2.3 \%$ & $+1-0.2$ \\
\hline $\begin{array}{l}\text { Usually worked } 1 \text { to } 14 \text { hours } \\
\text { per week }\end{array}$ & 22,139 & $+1-957$ & $4.2 \%$ & $+1-0.2$ & 8,719 & $+1-631$ & $3.2 \%$ & $+1-0.2$ & 13,420 & $+1-776$ & $5.1 \%$ & $+1-0.3$ \\
\hline 50 to 52 weeks & 6,993 & $+1-562$ & $1.3 \%$ & ++-0.1 & 2,640 & $+/-357$ & $1.0 \%$ & $+1-0.1$ & 4,353 & $+/-443$ & $1.7 \%$ & $+1-0.2$ \\
\hline 48 to 49 weeks & 501 & $+1-142$ & $0.1 \%$ & $+/-0.1$ & 130 & $+/-74$ & $0.0 \%$ & $+/-0.1$ & 371 & $+/-115$ & $0.1 \%$ & $+1-0.1$ \\
\hline 40 to 47 weeks & 1,735 & $+1-224$ & $0.3 \%$ & ++-0.1 & 536 & $+1-113$ & $0.2 \%$ & $+/-0.1$ & 1,199 & $+/-189$ & $0.5 \%$ & $+/-0.1$ \\
\hline 27 to 39 weeks & 2,957 & $+1-381$ & $0.6 \%$ & $+/-0.1$ & 982 & $+1-200$ & $0.4 \%$ & $+/-0.1$ & 1,975 & $+1-296$ & $0.8 \%$ & $+/-0.1$ \\
\hline 14 to 26 weeks & 3,148 & $+1-373$ & $0.6 \%$ & $+1-0.1$ & 1,241 & $+/-198$ & $0.5 \%$ & $+1-0.1$ & 1,907 & $+1-288$ & $0.7 \%$ & $+/-0.1$ \\
\hline 1 to 13 weeks & 6,805 & $+1-428$ & $1.3 \%$ & $+1-0.1$ & 3,190 & $+1-307$ & $1.2 \%$ & $+1-0.1$ & 3,615 & $+1-358$ & $1.4 \%$ & $+1-0.1$ \\
\hline Did not work & 80,997 & $+/-1,551$ & $15.2 \%$ & $+y-0.3$ & 34,021 & $+/-1,140$ & $12.5 \%$ & $+1-0.4$ & 46,976 & $+/-998$ & $18.0 \%$ & $+1-0.4$ \\
\hline $\begin{array}{l}\text { Mean usual hours worked for } \\
\text { workers }\end{array}$ & 39.7 & $+1-0.1$ & (X) & (X) & 42.8 & $+1-0.2$ & (X) & (X) & 36.2 & $+1-0.2$ & (X) & (X) \\
\hline $\begin{array}{l}\text { Median age of workers } 16 \text { to } 64 \\
\text { years }\end{array}$ & 39.8 & $+1-0.1$ & (X) & $(\mathrm{X})$ & 39.5 & $+1-0.3$ & (X) & $(\mathrm{X})$ & 40.1 & $+/-0.3$ & (X) & $(\mathrm{X})$ \\
\hline $\begin{array}{l}\text { Workers } 16 \text { to } 64 \text { years who } \\
\text { worked full-time, year-round }\end{array}$ & 298,888 & $+1-2,098$ & $66.2 \%$ & $+1-0.4$ & 170,758 & $+/-1,407$ & $71.9 \%$ & $+1-0.5$ & 128,130 & $+1-1,586$ & $60.0 \%$ & $+1-0.7$ \\
\hline
\end{tabular}

Explanation of Symbols:

1. An '**' entry in the margin of error column indicates that either no sample observations or too few sample observations were available to compute a standard error and thus the margin of error. A statistical test is not appropriate.

2. An '-' entry in the estimate column indicates that either no sample observations or too few sample observations were available to compute an estimate, or a ratio of medians cannot be calculated because one or both median estimates falls in the lowest interval or upper interval of an open-ended distribution.

3. An '-' following a median estimate means the median falls in the lowest interval of an open-ended distribution.

4. An ' + ' following a median estimate means the median falls in the upper interval of an open-ended distribution.

5. An '***' entry in the margin of error column indicates that the median falls in the lowest interval or upper interval of an open-ended distribution. A statistical test is not appropriate.

6. An '*****' entry in the margin of error column indicates that the estimate is controlled. A statistical test for sampling variability is not appropriate.

7. An ' $\mathrm{N}$ ' entry in the estimate and margin of error columns indicates that data for this geographic area cannot be displayed because the number of sample cases is too small.

8. An '(X)' means that the estimate is not applicable or not available.

Retrieved from https://factfinder.census.gov/ 
2006-2010 ACS 5-year Estimates, May 2011

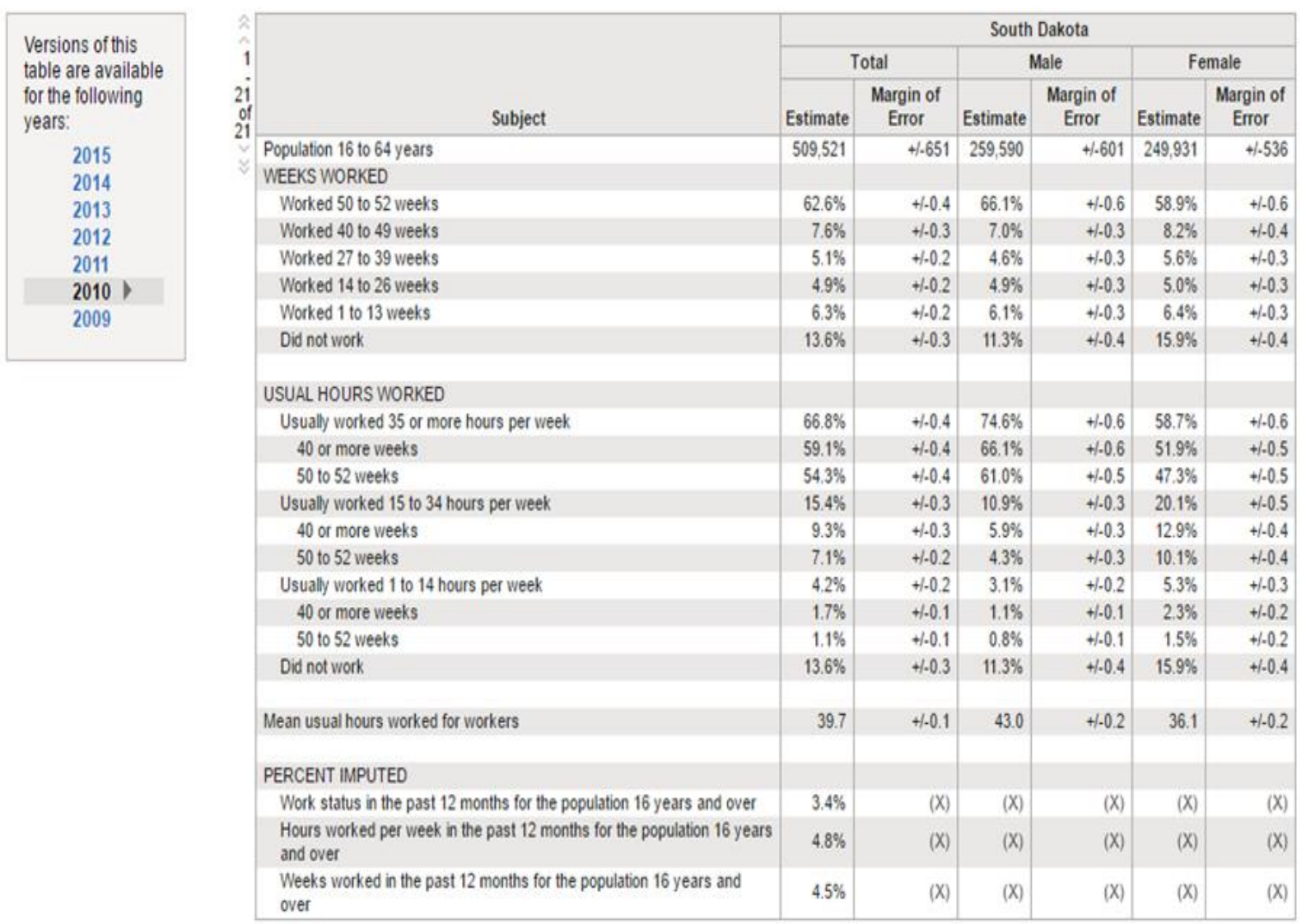

Source: U.S. Census Bureau, 2006-2010 American Community Survey

Explanation of Symbols:

1. An '**' entry in the margin of error column indicates that either no sample observations or too few sample observations were available to compute a standard error and thus the margin of error. A statistical test is not appropriate.

2. An '-' entry in the estimate column indicates that either no sample observations or too few sample observations were available to compute an estimate, or a ratio of medians cannot be calculated because one or both median estimates falls in the lowest interval or upper interval of an open-ended distribution.

3. An '-' following a median estimate means the median falls in the lowest interval of an open-ended distribution.

4. An ' + ' following a median estimate means the median falls in the upper interval of an open-ended distribution.

5. An '***' entry in the margin of error column indicates that the median falls in the lowest interval or upper interval of an open-ended distribution. A statistical test is not appropriate.

6. An '*****' entry in the margin of error column indicates that the estimate is controlled. A statistical test for sampling variability is not appropriate.

7. An ' $\mathrm{N}$ ' entry in the estimate and margin of error columns indicates that data for this geographic area cannot be displayed because the number of sample cases is too small.

8. An '(X)' means that the estimate is not applicable or not available.

Retrieved from https://factfinder.census.gov/ 


\section{APPENDIX D}

\section{Occupational Employment Statistics Query System, multiple occupations for one geographical area: South Dakota, May 2016 (reformatted)}

\begin{tabular}{|c|c|c|c|c|c|c|c|c|c|}
\hline Occupation (SOC code) & 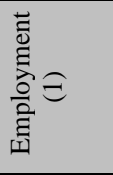 & 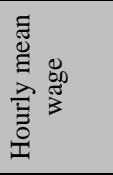 & 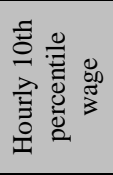 & 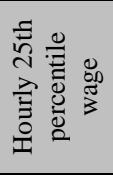 & 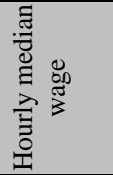 & 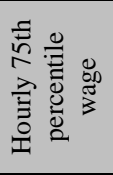 & 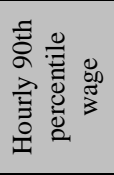 & 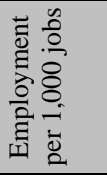 & 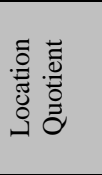 \\
\hline $\begin{array}{l}\text { Management Occupations } \\
(110000)\end{array}$ & 11,580 & 50.47 & 22.22 & 31.47 & 44.14 & 59.68 & 85.00 & 27.73 & 0.55 \\
\hline $\begin{array}{l}\text { Business and Financial } \\
\text { Operations Occupations } \\
\text { (130000) }\end{array}$ & 18,630 & 30.51 & 18.75 & 22.48 & 27.93 & 35.28 & 44.50 & 44.61 & 0.86 \\
\hline $\begin{array}{l}\text { Computer and Mathematical } \\
\text { Occupations (150000) }\end{array}$ & 7,830 & 30.74 & 17.56 & 22.06 & 28.63 & 37.42 & 47.16 & 18.75 & 0.63 \\
\hline $\begin{array}{l}\text { Architecture and Engineering } \\
\text { Occupations (170000) }\end{array}$ & 4,840 & 31.48 & 17.16 & 22.32 & 30.09 & 38.01 & 48.15 & 11.59 & 0.65 \\
\hline $\begin{array}{l}\text { Life, Physical, and Social } \\
\text { Science Occupations (190000) }\end{array}$ & 4,070 & 26.35 & 13.78 & 17.49 & 24.03 & 31.33 & 40.76 & 9.75 & 1.19 \\
\hline $\begin{array}{l}\text { Community and Social Service } \\
\text { Occupations (210000) }\end{array}$ & 6,550 & 19.03 & 11.96 & 15.24 & 18.26 & 22.43 & 27.38 & 15.68 & 1.09 \\
\hline Legal Occupations (230000) & 1,660 & 35.28 & 16.87 & 20.66 & 28.76 & 38.37 & 59.63 & 3.98 & 0.52 \\
\hline $\begin{array}{l}\text { Education, Training, and } \\
\text { Library Occupations (250000) }\end{array}$ & 24,170 & 20.29 & 11.02 & 14.21 & 18.66 & 24.29 & 30.69 & 57.90 & 0.94 \\
\hline $\begin{array}{l}\text { Arts, Design, Entertainment, } \\
\text { Sports, and Media Occupations } \\
\text { (270000) }\end{array}$ & 5,560 & 18.26 & 10.31 & 12.85 & 16.15 & 21.42 & 28.64 & 13.33 & 0.98 \\
\hline $\begin{array}{l}\text { Healthcare Practitioners and } \\
\text { Technical Occupations } \\
\text { (290000) }\end{array}$ & 29,800 & 33.29 & 15.10 & 19.83 & 25.70 & 35 & 56.31 & 71.37 & 1.20 \\
\hline $\begin{array}{l}\text { Healthcare Support } \\
\text { Occupations (310000) }\end{array}$ & 10,540 & 13.22 & 9.81 & 10.82 & 12.63 & 14.82 & 17.89 & 25.24 & 0.88 \\
\hline $\begin{array}{l}\text { Protective Service Occupations } \\
(330000)\end{array}$ & 6,470 & 18.92 & 10.43 & 12.99 & 17.21 & 22.92 & 29.95 & 15.50 & 0.64 \\
\hline $\begin{array}{l}\text { Food Preparation and Serving } \\
\text { Related Occupations (350000) }\end{array}$ & 41,410 & 10.53 & 8.88 & 9.13 & 9.62 & 11.34 & 13.58 & 99.18 & 1.07 \\
\hline $\begin{array}{l}\text { Building and Grounds } \\
\text { Cleaning and Maintenance } \\
\text { Occupations (370000) }\end{array}$ & 16,780 & 12.02 & 9.06 & 9.65 & 11.25 & 13.57 & 16.15 & 40.18 & 1.27 \\
\hline $\begin{array}{l}\text { Personal Care and Service } \\
\text { Occupations (390000) }\end{array}$ & 14,320 & 11.98 & 9.02 & 9.51 & 10.97 & 13.25 & 16.76 & 34.29 & 1.07 \\
\hline $\begin{array}{l}\text { Sales and Related Occupations } \\
(410000)\end{array}$ & 47,440 & 17.96 & 9.07 & 9.66 & 12.49 & 21.56 & 32.71 & 113.62 & 1.10 \\
\hline $\begin{array}{l}\text { Office and Administrative } \\
\text { Support Occupations (430000) }\end{array}$ & 64,720 & 14.75 & 9.75 & 11.47 & 14.00 & 17.28 & 20.94 & 155.02 & 0.99 \\
\hline
\end{tabular}

(continued) 


\section{APPENDIX D}

Occupational Employment Statistics Query System, multiple occupations for one geographical area: South Dakota, May 2016 (reformatted), continued

\begin{tabular}{|c|c|c|c|c|c|c|c|c|c|}
\hline Occupation (SOC code) & 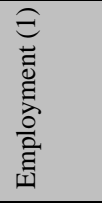 & 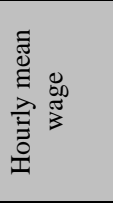 & 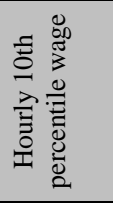 & 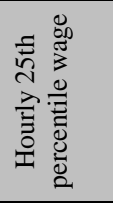 & 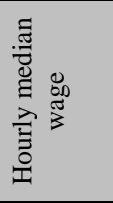 & 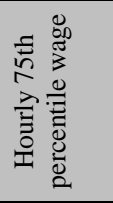 & 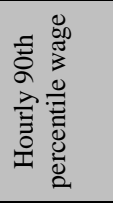 & 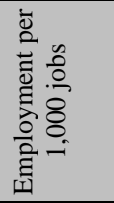 & 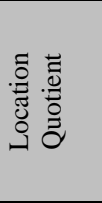 \\
\hline $\begin{array}{l}\text { Farming, Fishing, and Forestry } \\
\text { Occupations ( } 450000)\end{array}$ & 1,630 & 13.7 & 9.42 & 10.52 & 12.52 & 15.07 & 20.64 & 3.92 & 1.19 \\
\hline $\begin{array}{l}\text { Construction and Extraction } \\
\text { Occupations (470000) }\end{array}$ & 21,940 & 18.42 & 12.20 & 13.97 & 17.13 & 21.83 & 27.62 & 52.54 & 1.32 \\
\hline $\begin{array}{l}\text { Installation, Maintenance, and } \\
\text { Repair Occupations (490000) }\end{array}$ & 16,450 & 21.42 & 13.07 & 15.73 & 19.67 & 25.72 & 32.99 & 39.40 & 1.01 \\
\hline $\begin{array}{l}\text { Production Occupations } \\
\text { (510000) }\end{array}$ & 31,600 & 16.01 & 10.72 & 12.78 & 14.98 & 18.41 & 22.84 & 75.68 & 1.17 \\
\hline $\begin{array}{l}\text { Transportation and Material } \\
\text { Moving Occupations }(530000)\end{array}$ & 29,550 & 15.65 & 9.69 & 11.24 & 14.06 & 18.15 & 24.34 & 70.77 & 1.02 \\
\hline
\end{tabular}

Source: Bureau of Labor Statistics. (2016a). Occupational Employment Statistics (OES) data tables. Multiple occupations for one geographical area, South Dakota, May 2016 and May 2011. Retrieved from https://data.bls.gov/oes/ 


\section{APPENDIX E}

\section{Author's summary of IPUMS USA data for South Dakota 5-year ACS 2011-2015, adjusted to 2016 dollars}

\begin{tabular}{|c|c|c|c|c|c|}
\hline \multirow[b]{2}{*}{ OCC2010 } & \multirow[b]{2}{*}{$\begin{array}{l}\text { Category } \\
\text { Total }\end{array}$} & \multicolumn{2}{|c|}{$\begin{array}{c}\text { Single Earner } \\
(\$ 10.86 / \text { hour threshold })\end{array}$} & \multicolumn{2}{|c|}{$\begin{array}{l}\text { Household Income } \\
\text { (\$45,873/year } \\
\text { threshold) }\end{array}$} \\
\hline & & Count & $\begin{array}{l}\text { Percent } \\
\text { Under }\end{array}$ & Count & $\begin{array}{l}\text { Percent } \\
\text { Under }\end{array}$ \\
\hline Management - 0010-0430 & 74,694 & 16,825 & $22.53 \%$ & 39,911 & $53.43 \%$ \\
\hline Business \& Finance $-0500-0950$ & 21,162 & 3,179 & $15.02 \%$ & 11,448 & $54.10 \%$ \\
\hline Computer/Math - 1000-1240 & 8,830 & 1,166 & $13.20 \%$ & 3,741 & $42.37 \%$ \\
\hline $\begin{array}{l}\text { Architecture/Engineering }- \\
1560\end{array}$ & 7,855 & 819 & $10.43 \%$ & 2,478 & $31.55 \%$ \\
\hline Sciences - 1600-1980 & 5,128 & 888 & $17.32 \%$ & 2,939 & $57.31 \%$ \\
\hline $\begin{array}{l}\text { Community \& Social Services - 2000- } \\
2060\end{array}$ & 10,936 & 2,609 & $23.86 \%$ & 9,256 & $84.64 \%$ \\
\hline Legal - 2100-2150 & 4,022 & 784 & $19.49 \%$ & 1,841 & $45.77 \%$ \\
\hline Education $-2200-2550$ & 35,473 & 13,092 & $36.91 \%$ & 28,251 & $79.64 \%$ \\
\hline Arts/Entertainment - 2600-2920 & 9,283 & 4,475 & $48.21 \%$ & 7,440 & $80.15 \%$ \\
\hline $\begin{array}{l}\text { Healthcare Practitioners - } 3000- \\
3540\end{array}$ & 40,270 & 6,304 & $15.65 \%$ & 22,249 & $55.25 \%$ \\
\hline Healthcare Support - 3600-3650 & 18,079 & 11,261 & $62.29 \%$ & 17,502 & $96.81 \%$ \\
\hline Protective Services $-3700-3950$ & 10,759 & 3,788 & $35.21 \%$ & 8,130 & $75.56 \%$ \\
\hline Food Service $-4000-4150$ & 49,599 & 44,613 & $89.95 \%$ & 49,291 & $99.38 \%$ \\
\hline Building/Grounds - 4200-4250 & 27,694 & 19,642 & $70.93 \%$ & 26,368 & $95.21 \%$ \\
\hline Personal Care $-4300-4650$ & 28,978 & 19,142 & $66.06 \%$ & 26,701 & $92.14 \%$ \\
\hline Sales $-4700-4965$ & 73,325 & 35,968 & $49.05 \%$ & 54,737 & $74.65 \%$ \\
\hline $\begin{array}{l}\text { Office/Administrative Support - 5000- } \\
5940\end{array}$ & 98,839 & 41,087 & $41.57 \%$ & 88,622 & $89.66 \%$ \\
\hline Farming/Fishing $-6005-6130$ & 16,305 & 7,195 & $44.13 \%$ & 14,316 & $87.80 \%$ \\
\hline Construction $-6200-6940$ & 40,903 & 14,758 & $36.08 \%$ & 32,125 & $78.54 \%$ \\
\hline Installation/Maintenance - & 21,984 & 4,678 & $21.28 \%$ & 15,534 & $70.66 \%$ \\
\hline Production $-7700-8965$ & 46,690 & 16,724 & $35.82 \%$ & 40,360 & $86.44 \%$ \\
\hline Transportation $-9000-9750$ & 44,387 & 17,719 & $39.92 \%$ & 34,287 & $77.25 \%$ \\
\hline All Worker Totals & 695,195 & 286,716 & $41.24 \%$ & 537,527 & $77.32 \%$ \\
\hline All workers & 729,888 & & & & \\
\hline Military - 9800-9830 & 1,974 & $0.27 \%$ & & & \\
\hline Unemployed - 9920 & 32,719 & $4.48 \%$ & & & \\
\hline LABOR FORCE & 695,195 & & & & \\
\hline Wages, private & 442,610 & 195,637 & $44.20 \%$ & 355,804 & $80.39 \%$ \\
\hline Public workers, govt/nonprofit & 171,406 & 58,588 & $34.18 \%$ & 129,202 & $75.38 \%$ \\
\hline Self-employed & 79,134 & 30,872 & $39.01 \%$ & 50,733 & $64.11 \%$ \\
\hline Unpaid family worker & 2,045 & 1,619 & $79.17 \%$ & 1,788 & $87.43 \%$ \\
\hline & 695,195 & 286,716 & $41.24 \%$ & 537,527 & $77.32 \%$ \\
\hline
\end{tabular}

Source: Ruggles, S., Genadek, K., Goeken, R., Grover, J., and Sobek, M. (2015). Integrated Public Use Microdata Series: Version 6.0 [dataset]. Minneapolis: University of Minnesota. doi:10.18128/D010.V6.0

DOI: $10.17605 / O S F . I O / Q F 4 H Y$ 


\section{APPENDIX E}

Author's summary of IPUMS USA data for South Dakota

5-year ACS 2006-2010, adjusted to 2011 dollars

\begin{tabular}{|c|c|c|c|c|c|}
\hline \multirow[b]{2}{*}{ OCC2010 } & \multirow[b]{2}{*}{$\begin{array}{l}\text { Category } \\
\text { Total }\end{array}$} & \multicolumn{2}{|c|}{$\begin{array}{c}\text { Single Earner } \\
\text { (\$9.91/hour threshold) }\end{array}$} & \multicolumn{2}{|c|}{$\begin{array}{c}\text { Household Income } \\
(\$ 41,860 / \text { year } \\
\text { threshold })\end{array}$} \\
\hline & & Count & $\begin{array}{l}\text { Percent } \\
\text { Under }\end{array}$ & Count & $\begin{array}{l}\text { Percent } \\
\text { Under }\end{array}$ \\
\hline Management - 0010-0430 & 70,788 & 17,868 & $25.24 \%$ & 39,315 & $55.54 \%$ \\
\hline Business \& Finance $-0500-0950$ & 21,113 & 3,297 & $15.62 \%$ & 12,982 & $61.49 \%$ \\
\hline Computer/Math - 1000-1240 & 8,209 & 1,430 & $17.42 \%$ & 3,407 & $41.50 \%$ \\
\hline $\begin{array}{l}\text { Architecture/Engineering - } 1300- \\
1560\end{array}$ & 7,795 & 1,744 & $22.37 \%$ & 4,167 & $53.46 \%$ \\
\hline Sciences - 1600-1980 & 4,820 & 1,480 & $30.71 \%$ & 2,849 & $59.11 \%$ \\
\hline $\begin{array}{l}\text { Community \& Social Services - 2000- } \\
2060\end{array}$ & 12,133 & 2,328 & $19.19 \%$ & 8,815 & $72.65 \%$ \\
\hline Legal - 2100-2150 & 4,133 & 600 & $14.52 \%$ & 2,184 & $52.84 \%$ \\
\hline Education - 2200-2550 & 36,779 & 12,047 & $32.76 \%$ & 29,693 & $80.73 \%$ \\
\hline Arts/Entertainment - 2600-2920 & 9,316 & 4,870 & $52.28 \%$ & 7,999 & $85.86 \%$ \\
\hline $\begin{array}{l}\text { Healthcare Practitioners - } 3000- \\
3540\end{array}$ & 34,683 & 5,397 & $15.56 \%$ & 17,748 & $51.17 \%$ \\
\hline Healthcare Support - 3600-3650 & 18,315 & 11,388 & $62.18 \%$ & 17,815 & $97.27 \%$ \\
\hline Protective Services - 3700-3950 & 11,639 & 4,178 & $35.90 \%$ & 8,625 & $74.10 \%$ \\
\hline Food Service $-4000-4150$ & 40,632 & 35,643 & $87.72 \%$ & 40,104 & $98.70 \%$ \\
\hline Building/Grounds $-4200-4250$ & 22,853 & 16,489 & $72.15 \%$ & 21,989 & $96.22 \%$ \\
\hline Personal Care - 4300-4650 & 22,482 & 16,032 & $71.31 \%$ & 21,327 & $94.86 \%$ \\
\hline Sales - 4700-4965 & 67,836 & 33,756 & $49.76 \%$ & 52,537 & $77.45 \%$ \\
\hline $\begin{array}{l}\text { Office/Administrative Support - 5000- } \\
5940\end{array}$ & 98,378 & 40,913 & $41.59 \%$ & 88,758 & $90.22 \%$ \\
\hline Farming/Fishing - 6005-6130 & 17,959 & 11,738 & $65.36 \%$ & 16,639 & $92.65 \%$ \\
\hline Construction $-6200-6940$ & 36,612 & 13,121 & $35.84 \%$ & 28,285 & $77.26 \%$ \\
\hline Installation/Maintenance - & 23,756 & 6,742 & $28.38 \%$ & 17,143 & $72.16 \%$ \\
\hline Production $-7700-8965$ & 45,914 & 15,375 & $33.49 \%$ & 40,402 & $87.99 \%$ \\
\hline Transportation - 9000-9750 & 39,028 & 17,025 & $43.62 \%$ & 31,615 & $81.01 \%$ \\
\hline All Worker Totals & 655,173 & 273,461 & $41.74 \%$ & 514,398 & $78.51 \%$ \\
\hline All workers & 685,277 & & & & \\
\hline Military $-9800-9830$ & 1,582 & $0.23 \%$ & & & \\
\hline Unemployed - 9920 & 28,522 & $4.16 \%$ & & & \\
\hline LABOR FORCE & 655,173 & & & & \\
\hline Wages, private & 407,667 & 182,466 & $44.76 \%$ & 334,696 & $82.10 \%$ \\
\hline Public workers, govt/nonprofit & 167,532 & 57,268 & $34.18 \%$ & 124,520 & $74.33 \%$ \\
\hline Self-employed & 77,069 & 32,242 & $41.84 \%$ & 53,202 & $69.03 \%$ \\
\hline Unpaid family worker & 2,905 & 1,485 & $51.12 \%$ & 2,188 & $75.32 \%$ \\
\hline & 655,173 & 273,461 & $41.74 \%$ & 514,606 & $78.55 \%$ \\
\hline
\end{tabular}

Source: Ruggles, S., Genadek, K., Goeken, R., Grover, J., and Sobek, M. (2015). Integrated Public Use Microdata Series: Version 6.0 [dataset]. Minneapolis: University of Minnesota. doi:10.18128/D010.V6.0

DOI: $10.17605 / O S F . I O / Q F 4 H Y$ 


\section{APPENDIX F}

\section{Author's summary of South Dakota Workers by Age Groups, Occupations, and Income Levels, 2011}

\begin{tabular}{|c|c|c|c|c|c|}
\hline \multicolumn{6}{|c|}{ Workers by Age, Occupation, and Income Levels - 2011} \\
\hline Sum of Workers & \multicolumn{3}{|c|}{ Household Level $(<\$ 41,860 / \mathbf{y r})$} & \multirow[b]{2}{*}{$\begin{array}{c}\text { Higher Wage } \\
\text { (At least } \\
\$ 41,860 / \mathbf{y r}) \\
\end{array}$} & \multirow[b]{2}{*}{ Grand Total } \\
\hline AGE GROUPS & $\underset{(<\$ 20,930 / y r)}{\text { Low Wage }}$ & $\begin{array}{c}\text { Between Low Wage \& } \\
\text { Household } \\
(\$ 20,930-\$ 41,859 / \mathrm{yr}) \\
\end{array}$ & Total & & \\
\hline $16-29$ & 82,132 & 34,475 & 119,606 & 7,040 & 126,646 \\
\hline Management & 2,585 & 2,036 & 4,621 & 760 & 5,381 \\
\hline Business \& Finance & 872 & 1,097 & 1,969 & 424 & 2,393 \\
\hline Computer/Math & 490 & 314 & 804 & 395 & 1,199 \\
\hline Architecture/Engineering & 598 & 541 & 1,138 & 441 & 1,579 \\
\hline Sciences & 660 & 228 & 888 & 114 & 1,003 \\
\hline Community \& Social Services & 263 & 841 & 1,103 & 50 & 1,154 \\
\hline Legal & 71 & 182 & 254 & 89 & 342 \\
\hline Education & 2,583 & 2,140 & 4,723 & 67 & 4,790 \\
\hline Arts/Entertainment & 1,357 & 427 & 1,784 & 48 & 1,832 \\
\hline Healthcare Practitioners & 1,599 & 1,730 & 3,329 & 1,122 & 4,451 \\
\hline Healthcare Support & 3,572 & 1,085 & 4,658 & & 4,658 \\
\hline Protective Services & 1,615 & 903 & 2,518 & 316 & 2,834 \\
\hline Food Services & 15,483 & 936 & 16,419 & 94 & 16,513 \\
\hline Building/Grounds & 3,906 & 363 & 4,269 & 65 & 4,334 \\
\hline Personal Care & 4,976 & 846 & 5,823 & 95 & 5,918 \\
\hline Sales & 11,830 & 2,702 & 14,533 & 734 & 15,266 \\
\hline Office/Admin & 10,329 & 5,825 & 16,153 & 657 & 16,810 \\
\hline Farming/Fishing & 5,164 & 1,162 & 6,326 & 93 & 6,418 \\
\hline Construction & 4,771 & 2,828 & 7,600 & 413 & 8,013 \\
\hline Install/Maintenance & 2,059 & 2,408 & 4,468 & 296 & 4,764 \\
\hline Production & 4,591 & 4,397 & 8,988 & 438 & 9,426 \\
\hline Transportation & 5,756 & 1,482 & 7,238 & 328 & 7,567 \\
\hline 30-39 & 19,476 & 32,531 & 52,007 & 20,278 & 72,285 \\
\hline Management & 1,084 & 2,809 & 3,893 & 3,968 & 7,861 \\
\hline Business \& Finance & 342 & 1,549 & 1,892 & 1,515 & 3,407 \\
\hline Computer/Math & 101 & 452 & 553 & 1,153 & 1,705 \\
\hline Architecture/Engineering & 155 & 312 & 466 & 433 & 899 \\
\hline Sciences & 85 & 384 & 469 & 499 & 967 \\
\hline Community \& Social Services & 278 & 1,050 & 1,328 & 409 & 1,736 \\
\hline Legal & 98 & 360 & 458 & 258 & 716 \\
\hline Education & 1,004 & 2,428 & 3,432 & 419 & 3,851 \\
\hline Arts/Entertainment & 506 & 733 & 1,239 & 161 & 1,400 \\
\hline Healthcare Practitioners & 544 & 1,823 & 2,367 & 2,524 & 4,891 \\
\hline Healthcare Support & 955 & 710 & 1,664 & 32 & 1,696 \\
\hline Protective Services & 359 & 749 & 1,108 & 520 & 1,628 \\
\hline Food Services & 1,514 & 533 & 2,047 & 58 & 2,105 \\
\hline Building/Grounds & 1,509 & 234 & 1,743 & 52 & 1,795 \\
\hline
\end{tabular}




\section{APPENDIX F}

Author's summary of South Dakota Workers by Age Groups, Occupations, and Income Levels 2011 (continued)

\begin{tabular}{|c|c|c|c|c|c|}
\hline \multicolumn{6}{|c|}{ Workers by Age, Occupation, and Income Levels - 2011} \\
\hline Sum of Workers & \multicolumn{3}{|c|}{ Household Level $(<\$ 41,860 / y r)$} & \multirow[b]{2}{*}{$\begin{array}{l}\text { Higher Wage } \\
\text { (At least } \\
\$ 41,860 / y r) \\
\end{array}$} & \multirow[b]{2}{*}{ Grand Total } \\
\hline AGE GROUPS & $\begin{array}{c}\text { Low Wage } \\
(<\$ 20,930 / y r)\end{array}$ & $\begin{array}{c}\text { Between Low Wage \& } \\
\text { Household } \\
(\$ 20,930-\$ 41,859 / \mathbf{y r}) \\
\end{array}$ & Total & & \\
\hline \multicolumn{6}{|l|}{ 30-39 (continued) } \\
\hline Personal Care & 1,392 & 752 & 2,144 & 273 & 2,417 \\
\hline Sales & 2,298 & 2,106 & 4,403 & 2,468 & 6,872 \\
\hline Office/Admin & 3,334 & 5,184 & 8,519 & 649 & 9,167 \\
\hline Farming/Fishing & 476 & 721 & 1,197 & 112 & 1,309 \\
\hline Construction & 918 & 2,485 & 3,403 & 1,331 & 4,734 \\
\hline Install/Maintenance & 609 & 1,379 & 1,988 & 1,385 & 3,373 \\
\hline Production & 1,014 & 3,561 & 4,575 & 1,096 & 5,670 \\
\hline Transportation & 902 & 2,218 & 3,120 & 966 & 4,085 \\
\hline $40-49$ & 19,961 & 35,012 & $\mathbf{5 4 , 9 7 3}$ & 25,366 & 80,340 \\
\hline Management & 2,056 & 3,262 & 5,317 & 5,815 & 11,132 \\
\hline Business \& Finance & 194 & 1,608 & 1,802 & 1,364 & 3,166 \\
\hline Computer/Math & 37 & 210 & 247 & 712 & 959 \\
\hline Architecture/Engineering & 82 & 294 & 376 & 667 & 1,043 \\
\hline Sciences & 27 & 138 & 165 & 324 & 489 \\
\hline Community \& Social Services & 346 & 679 & 1,025 & 513 & 1,538 \\
\hline Legal & 72 & 230 & 301 & 295 & 597 \\
\hline Education & 1,323 & 2,536 & 3,859 & 1,097 & 4,956 \\
\hline Arts/Entertainment & 359 & 331 & 690 & 348 & 1,038 \\
\hline Healthcare Practitioners & 352 & 1,613 & 1,965 & 3,044 & 5,009 \\
\hline Healthcare Support & 973 & 779 & 1,752 & 25 & 1,776 \\
\hline Protective Services & 186 & 491 & 677 & 718 & 1,395 \\
\hline Food Services & 1,434 & 676 & 2,110 & 110 & 2,219 \\
\hline Building/Grounds & 1,564 & 819 & 2,383 & 61 & 2,444 \\
\hline Personal Care & 931 & 953 & 1,884 & 225 & 2,109 \\
\hline Sales & 1,755 & 2,819 & 4,574 & 2,621 & 7,195 \\
\hline Office/Admin & 3,565 & 8,305 & 11,870 & 2,117 & 13,987 \\
\hline Farming/Fishing & 547 & 379 & 927 & 224 & 1,151 \\
\hline Construction & 1,069 & 1,809 & 2,877 & 1,627 & 4,504 \\
\hline Install/Maintenance & 592 & 1,396 & 1,989 & 1,118 & 3,107 \\
\hline Production & 1,419 & 3,868 & 5,287 & 1,065 & 6,352 \\
\hline Transportation & 1,078 & 1,817 & 2,895 & 1,277 & 4,172 \\
\hline
\end{tabular}

(continued) 


\section{APPENDIX G}

Author's summary of South Dakota Workers by Age Groups, Occupations, and Income Levels 2011 (continued)

\begin{tabular}{|c|c|c|c|c|c|}
\hline \multicolumn{6}{|c|}{ Workers by Age, Occupation, and Income Levels - 2011} \\
\hline Sum of Workers & \multicolumn{3}{|c|}{ Household Level $(<\$ 41,860 / y r)$} & \multirow[b]{2}{*}{$\begin{array}{l}\text { Higher Wage } \\
\text { (At least } \\
\$ 41,860 / y r) \\
\end{array}$} & \multirow[b]{2}{*}{$\begin{array}{c}\text { Grand } \\
\text { Total }\end{array}$} \\
\hline AGE GROUPS & $\begin{array}{c}\text { Low Wage } \\
(<\$ 20,930 / y r)\end{array}$ & $\begin{array}{c}\text { Between Low Wage \& } \\
\text { Household } \\
(\$ 20,930-\$ 41,859 / \mathrm{yr}) \\
\end{array}$ & Total & & \\
\hline $50-64$ & 27,734 & 38,670 & 66,404 & 29,166 & 95,570 \\
\hline Management & 3,223 & 3,980 & 7,204 & 7,269 & 14,473 \\
\hline Business \& Finance & 372 & 1,401 & 1,772 & 1,460 & 3,232 \\
\hline Computer/Math & 229 & 172 & 401 & 571 & 972 \\
\hline Architecture/Engineering & 189 & 306 & 494 & 634 & 1,128 \\
\hline Sciences & 95 & 64 & 158 & 245 & 403 \\
\hline Community \& Social Services & 267 & 1,112 & 1,379 & 815 & 2,193 \\
\hline Legal & 59 & 157 & 216 & 460 & 675 \\
\hline Education & 1,736 & 3,425 & 5,161 & 2,578 & 7,739 \\
\hline Arts/Entertainment & 382 & 314 & 696 & 182 & 878 \\
\hline Healthcare Practitioners & 551 & 2,095 & 2,646 & 3,260 & 5,905 \\
\hline Healthcare Support & 1,170 & 1,212 & 2,382 & 243 & 2,626 \\
\hline Protective Services & 279 & 474 & 753 & 253 & 1,006 \\
\hline Food Services & 2,145 & 316 & 2,462 & 55 & 2,517 \\
\hline Building/Grounds & 1,856 & 1,755 & 3,611 & 327 & 3,937 \\
\hline Personal Care & 1,709 & 579 & 2,288 & 84 & 2,372 \\
\hline Sales & 2,961 & 3,176 & 6,137 & 3,130 & 9,267 \\
\hline Office/Admin & 5,477 & 8,668 & 14,145 & 2,245 & 16,389 \\
\hline Farming/Fishing & 527 & 592 & 1,119 & 345 & 1,464 \\
\hline Construction & 839 & 1,877 & 2,716 & 1,562 & 4,278 \\
\hline Install/Maintenance & 569 & 1,115 & 1,684 & 996 & 2,680 \\
\hline Production & 1,767 & 2,982 & 4,749 & 668 & 5,417 \\
\hline Transportation & 1,334 & 2,899 & 4,233 & 1,786 & 6,019 \\
\hline $65-94$ & 11,597 & 3,843 & 15,440 & 2,399 & 17,839 \\
\hline Management & 1,761 & 768 & 2,529 & 1,051 & 3,580 \\
\hline Business \& Finance & 197 & 150 & 346 & 110 & 456 \\
\hline Computer/Math & & 37 & 37 & 47 & 85 \\
\hline Architecture/Engineering & 22 & & 22 & & 22 \\
\hline Sciences & 21 & 6 & 27 & & 27 \\
\hline Community \& Social Services & 243 & 206 & 449 & 202 & 651 \\
\hline Legal & 59 & 21 & 80 & 66 & 146 \\
\hline Education & 574 & 48 & 622 & 86 & 707 \\
\hline Arts/Entertainment & 314 & 70 & 384 & 52 & 435 \\
\hline Healthcare Practitioners & 189 & 141 & 331 & 200 & 530 \\
\hline Healthcare Support & 156 & 65 & 221 & & 221 \\
\hline Protective Services & 65 & 47 & 113 & & 113 \\
\hline Food Services & 787 & 212 & 999 & & 999 \\
\hline Building/Grounds & 1,048 & 126 & 1,174 & 13 & 1,187 \\
\hline
\end{tabular}

(continued)

DOI: $10.17605 / O S F . I O / Q F 4 H Y$ 


\section{APPENDIX F}

Author's summary of South Dakota Workers by Age Groups, Occupations, and Income Levels 2011 (continued)

\begin{tabular}{|c|c|c|c|c|c|}
\hline \multicolumn{6}{|c|}{ Workers by Age, Occupation, and Income Levels - 2011} \\
\hline Sum of Workers & \multicolumn{3}{|c|}{ Household Level $(<\$ 41,860 / y r)$} & \multirow[b]{2}{*}{$\begin{array}{c}\text { Higher Wage } \\
\text { (At least } \\
\$ 41,860 / \mathrm{yr}) \\
\end{array}$} & \multirow[b]{2}{*}{ Grand Total } \\
\hline AGE GROUPS & $\underset{(<\$ 20,930 / y r)}{\text { Low Wage }}$ & $\begin{array}{c}\text { Between Low Wage \& } \\
\text { Household } \\
(\$ 20,930-\$ 41,859 / y r) \\
\end{array}$ & Total & & \\
\hline \multicolumn{6}{|l|}{ 65-94 (continued) } \\
\hline Personal Care & 599 & 43 & 643 & 16 & 658 \\
\hline Sales & 1,388 & 454 & 1,842 & 216 & 2,058 \\
\hline Office/Admin & 1,817 & 694 & 2,511 & 99 & 2,610 \\
\hline Farming/Fishing & 321 & 83 & 405 & 17 & 421 \\
\hline Construction & 267 & 90 & 357 & 58 & 415 \\
\hline Install/Maintenance & 211 & 60 & 271 & 44 & 315 \\
\hline Production & 424 & 192 & 616 & 37 & 653 \\
\hline Transportation & 1,134 & 329 & 1,463 & 86 & 1,549 \\
\hline Grand Total & 163,900 & 144,543 & $\mathbf{3 0 8 , 4 3 1}$ & 84,249 & 392,680 \\
\hline
\end{tabular}




\section{APPENDIX F}

Author's summary of South Dakota Workers by Age Groups, Occupations, and Income Levels 2016 (continued)

\begin{tabular}{|c|c|c|c|c|c|}
\hline \multicolumn{6}{|c|}{ Workers by Age, Occupation, and Income Levels - 2016} \\
\hline Sum of Workers & \multicolumn{3}{|c|}{ Household Level $(<\$ 45,873 / \mathbf{y r})$} & \multirow[b]{2}{*}{$\begin{array}{l}\text { Higher Wage } \\
\text { (At least } \\
\$ 45,873 / \mathrm{yr} \text { ) } \\
\end{array}$} & \multirow[b]{2}{*}{ Grand Total } \\
\hline AGE GROUPS & $\underset{(<\$ 22,936 / y r)}{\text { Low Wage }}$ & $\begin{array}{c}\text { Between Low Wage \& } \\
\text { Household } \\
(\$ 22,936-\$ 45,872 / y r) \\
\end{array}$ & Total & & \\
\hline $16-29$ & 88,019 & 35,303 & 123,321 & 9,156 & 132,477 \\
\hline Management & 1,645 & 2,795 & 4,440 & 720 & 5,159 \\
\hline Business \& Finance & 592 & 1,024 & 1,616 & 139 & 1,756 \\
\hline Computer/Math & 548 & 548 & 1,096 & 380 & 1,476 \\
\hline Architecture/Engineering & 341 & 499 & 840 & 598 & 1,437 \\
\hline Sciences & 354 & 631 & 986 & 144 & 1,130 \\
\hline Community \& Social Services & 442 & 815 & 1,257 & 4 & 1,261 \\
\hline Legal & 214 & 49 & 263 & 97 & 360 \\
\hline Education & 2,586 & 1,741 & 4,327 & 43 & 4,370 \\
\hline Arts/Entertainment & 1,187 & 353 & 1,541 & 263 & 1,804 \\
\hline Healthcare Practitioners & 1,751 & 2,938 & 4,689 & 1,110 & 5,799 \\
\hline Healthcare Support & 3,461 & 892 & 4,353 & 160 & 4,513 \\
\hline Protective Services & 1,485 & 753 & 2,238 & 169 & 2,407 \\
\hline Food Services & 18,851 & 1,022 & 19,872 & 70 & 19,943 \\
\hline Building/Grounds & 4,452 & 739 & 5,191 & 130 & 5,321 \\
\hline Personal Care & 6,343 & 1,261 & 7,604 & 453 & 8,057 \\
\hline Sales & 12,892 & 2,192 & 15,084 & 1,011 & 16,095 \\
\hline Office/Admin & 11,654 & 5,013 & 16,667 & 220 & 16,887 \\
\hline Farming/Fishing & 2,750 & 2,020 & 4,771 & 254 & 5,025 \\
\hline Construction & 5,153 & 3,091 & 8,243 & 1,088 & 9,331 \\
\hline Install/Maintenance & 1,516 & 2,053 & 3,569 & 610 & 4,180 \\
\hline Production & 4,706 & 2,835 & 7,541 & 790 & 8,331 \\
\hline Transportation & 5,095 & 2,037 & 7,132 & 705 & 7,837 \\
\hline 30-39 & 23,847 & 36,215 & 60,061 & 21,627 & 81,689 \\
\hline Management & 2,623 & 3,626 & 6,249 & 3,957 & 10,207 \\
\hline Business \& Finance & 412 & 1,186 & 1,598 & 1,737 & 3,335 \\
\hline Computer/Math & 37 & 437 & 473 & 850 & 1,324 \\
\hline Architecture/Engineering & 90 & 214 & 304 & 962 & 1,265 \\
\hline Sciences & 61 & 465 & 526 & 329 & 855 \\
\hline Community \& Social Services & 306 & 911 & 1,217 & 90 & 1,307 \\
\hline Legal & 23 & 163 & 186 & 276 & 461 \\
\hline Education & 1,142 & 2,499 & 3,641 & 870 & 4,511 \\
\hline Arts/Entertainment & 535 & 688 & 1,223 & 225 & 1,448 \\
\hline Healthcare Practitioners & 587 & 2,389 & 2,976 & 3,258 & 6,234 \\
\hline Healthcare Support & 1,536 & 726 & 2,262 & 86 & 2,348 \\
\hline Protective Services & 184 & 638 & 823 & 426 & 1,249 \\
\hline Food Services & 3,014 & 594 & 3,608 & & 3,608 \\
\hline Building/Grounds & 1,617 & 855 & 2,472 & 162 & 2,634 \\
\hline
\end{tabular}

DOI: $10.17605 / \mathrm{OSF} . \mathrm{IO} / \mathrm{QF} 4 \mathrm{HY}$ 


\section{APPENDIX F}

Author's summary of South Dakota Workers by Age Groups, Occupations, and Income Levels 2016 (continued)

\begin{tabular}{|c|c|c|c|c|c|}
\hline \multicolumn{6}{|c|}{ Workers by Age, Occupation, and Income Levels - 2016} \\
\hline Sum of Workers & \multicolumn{3}{|c|}{ Household Level $(<\$ 45,873 / \mathrm{yr})$} & \multirow[b]{2}{*}{$\begin{array}{c}\text { Higher Wage } \\
\text { (At least } \\
\$ 45,873 / y r) \\
\end{array}$} & \multirow[b]{2}{*}{ Grand Total } \\
\hline AGE GROUPS & $\begin{array}{c}\text { Low Wage } \\
(<\$ 22,936 / y r)\end{array}$ & $\begin{array}{c}\text { Between Low Wage \& } \\
\text { Household } \\
(\$ 22,936-\$ 45,872 / y r) \\
\end{array}$ & Total & & \\
\hline \multicolumn{6}{|l|}{ 30-39 (continued) } \\
\hline Personal Care & 1,394 & 984 & 2,378 & 311 & 2,688 \\
\hline Sales & 2,360 & 2,487 & 4,847 & 2,439 & 7,286 \\
\hline Office/Admin & 3,196 & 6,573 & 9,769 & 1,287 & 11,055 \\
\hline Farming/Fishing & 494 & 837 & 1,311 & 291 & 1,622 \\
\hline Construction & 1,085 & 2,662 & 3,747 & 1,099 & 4,846 \\
\hline Install/Maintenance & 289 & 1,319 & 1,608 & 988 & 2,596 \\
\hline Production & 1,723 & 4,052 & 5,775 & 600 & 6,375 \\
\hline Transportation & 1,137 & 1,912 & 3,049 & 1,384 & 4,432 \\
\hline $40-49$ & 18,305 & 31,077 & 49,381 & 25,684 & 75,065 \\
\hline Management & 1,984 & 2,705 & 4,688 & 6,096 & 10,784 \\
\hline Business \& Finance & 159 & 1,130 & 1,289 & 1,510 & 2,799 \\
\hline Computer/Math & 12 & 273 & 285 & 799 & 1,085 \\
\hline Architecture/Engineering & 7 & 86 & 92 & 837 & 930 \\
\hline Sciences & 25 & 21 & 46 & 331 & 377 \\
\hline Community \& Social Services & 312 & 590 & 903 & 297 & 1,200 \\
\hline Legal & 16 & 205 & 221 & 272 & 493 \\
\hline Education & 1,017 & 2,017 & 3,035 & 1,173 & 4,208 \\
\hline Arts/Entertainment & 194 & 312 & 506 & 279 & 784 \\
\hline Healthcare Practitioners & 485 & 1,716 & 2,201 & 2,767 & 4,968 \\
\hline Healthcare Support & 539 & 874 & 1,414 & 49 & 1,463 \\
\hline Protective Services & 91 & 431 & 521 & 610 & 1,132 \\
\hline Food Services & 1,747 & 589 & 2,336 & 38 & 2,374 \\
\hline Building/Grounds & 1,844 & 673 & 2,517 & 197 & 2,714 \\
\hline Personal Care & 996 & 1,163 & 2,160 & 244 & 2,404 \\
\hline Sales & 1,780 & ,485 & 4,266 & 3,156 & 7,422 \\
\hline Office/Admin & 2,771 & 6,482 & 9,252 & 1,897 & 11,149 \\
\hline Farming/Fishing & 332 & 621 & 953 & 128 & 1,081 \\
\hline Construction & 847 & 2,012 & 2,859 & 1,172 & 4,032 \\
\hline Install/Maintenance & 282 & 1,353 & 1,635 & 1,064 & 2,699 \\
\hline Production & 1,563 & 3,041 & 4,605 & 1,106 & 5,711 \\
\hline Transportation & 1,300 & 2,296 & 3,596 & 1,660 & 5,257 \\
\hline
\end{tabular}




\section{APPENDIX F}

Author's summary of South Dakota Workers by Age Groups, Occupations, and Income Levels 2016 (continued)

\begin{tabular}{|c|c|c|c|c|c|}
\hline \multicolumn{6}{|c|}{ Workers by Age, Occupation, and Income Levels - 2016} \\
\hline Sum of Workers & \multicolumn{3}{|c|}{ Household Level $(<\$ 45,873 / \mathrm{yr})$} & \multirow[b]{2}{*}{$\begin{array}{l}\text { Higher Wage } \\
\text { (At least } \\
\$ 45,873 / \mathrm{yr} \text { ) } \\
\end{array}$} & \multirow[b]{2}{*}{ Grand Total } \\
\hline AGE GROUPS & $\underset{(<\$ 22,936 / y r)}{\text { Low Wage }}$ & $\begin{array}{c}\text { Between Low Wage \& } \\
\text { Household } \\
(\$ 22,936-\$ 45,872 / y r) \\
\end{array}$ & Total & & \\
\hline $50-64$ & 29,675 & 42,698 & 72,373 & 34,172 & 106,546 \\
\hline Management & 2,809 & 3,872 & 6,681 & 8,708 & 15,389 \\
\hline Business \& Finance & 593 & 1,499 & 2,092 & 2,093 & 4,184 \\
\hline Computer/Math & 84 & 274 & 359 & 996 & 1,354 \\
\hline Architecture/Engineering & 17 & 193 & 210 & 730 & 941 \\
\hline Sciences & 91 & 91 & 181 & 469 & 650 \\
\hline Community \& Social Services & 291 & 1,557 & 1,847 & 470 & 2,317 \\
\hline Legal & 207 & 190 & 398 & 595 & 993 \\
\hline Education & 1,582 & 2,578 & 4,160 & 2,035 & 6,195 \\
\hline Arts/Entertainment & 616 & 408 & 1,024 & 335 & 1,359 \\
\hline Healthcare Practitioners & 691 & 2,275 & 2,966 & 3,468 & 6,434 \\
\hline Healthcare Support & 864 & 1,171 & 2,035 & 51 & 2,086 \\
\hline Protective Services & 392 & 750 & 1,142 & 327 & 1,469 \\
\hline Food Services & 2,389 & 515 & 2,903 & 49 & 2,953 \\
\hline Building/Grounds & 3,059 & 1,372 & 4,431 & 255 & 4,687 \\
\hline Personal Care & 1,884 & 909 & 2,793 & 303 & 3,097 \\
\hline Sales & 2,828 & 3,698 & 6,526 & 3,979 & 10,505 \\
\hline Office/Admin & 5,299 & 9,428 & 14,726 & 2,581 & 17,307 \\
\hline Farming/Fishing & 551 & 735 & 1,287 & 462 & 1,749 \\
\hline Construction & 1,524 & 2,497 & 4,021 & 1,752 & 5,773 \\
\hline Install/Maintenance & 308 & 1,592 & 1,899 & 1,131 & 3,030 \\
\hline Production & 1,608 & 3,797 & 5,405 & 1,256 & 6,661 \\
\hline Transportation & 1,989 & 3,296 & 5,285 & 2,126 & 7,412 \\
\hline $65-95$ & 12,359 & 5,347 & 17,706 & 4,058 & 21,764 \\
\hline Management & 1,044 & 868 & 1,912 & 1,410 & 3,323 \\
\hline Business \& Finance & 153 & 127 & 280 & 356 & 636 \\
\hline Computer/Math & 20 & 14 & 34 & 31 & 65 \\
\hline Architecture/Engineering & 38 & 4 & 42 & 103 & 145 \\
\hline Sciences & 2 & 24 & 26 & 42 & 68 \\
\hline Community \& Social Services & 216 & 119 & 335 & 148 & 483 \\
\hline Legal & 11 & 28 & 38 & 70 & 108 \\
\hline Education & 1,535 & 269 & 1,804 & 217 & 2,022 \\
\hline Arts/Entertainment & 156 & 19 & 175 & 5 & 180 \\
\hline Healthcare Practitioners & 273 & 258 & 531 & 220 & 751 \\
\hline Healthcare Support & 363 & 85 & 448 & & 448 \\
\hline Protective Services & 123 & 36 & 159 & 46 & 205 \\
\hline Food Services & 795 & 90 & 885 & 27 & 912 \\
\hline Building/Grounds & 825 & 401 & 1,225 & 2 & 1,277 \\
\hline
\end{tabular}

(continued)

DOI: $10.17605 / O S F . I O / Q F 4 H Y$ 


\section{APPENDIX F}

Author's summary of South Dakota Workers by Age Groups, Occupations, and Income Levels 2016 (continued)

\begin{tabular}{|c|c|c|c|c|c|}
\hline \multicolumn{6}{|c|}{ Workers by Age, Occupation, and Income Levels - 2016} \\
\hline Sum of Workers & \multicolumn{3}{|c|}{ Household Level $(<\$ 45,873 / y r)$} & \multirow[b]{2}{*}{$\begin{array}{c}\text { Higher Wage } \\
\text { (At least } \\
\$ 45,873 / y r)\end{array}$} & \multirow[b]{2}{*}{$\begin{array}{c}\text { Grand } \\
\text { Total }\end{array}$} \\
\hline AGE GROUPS & $\begin{array}{c}\text { Low Wage } \\
(<\$ 22,936 / y r)\end{array}$ & $\begin{array}{c}\text { Between Low Wage \& } \\
\text { Household } \\
(\$ 22,936-\$ 45,872 / y r)\end{array}$ & Total & & \\
\hline \multicolumn{6}{|l|}{ 65-94 (continued) } \\
\hline Personal Care & 879 & 223 & 1,102 & 57 & 1,159 \\
\hline Sales & 1,742 & 411 & 2,153 & 579 & 2,732 \\
\hline Office/Admin & 1,758 & 1,054 & 2,812 & 153 & 2,965 \\
\hline Farming/Fishing & 193 & 64 & 257 & 59 & 316 \\
\hline Construction & 254 & 169 & 423 & 161 & 584 \\
\hline Install/Maintenance & 416 & 203 & 619 & 80 & 699 \\
\hline Production & 443 & 471 & 914 & 50 & 964 \\
\hline Transportation & 1,121 & 410 & 1,530 & 192 & 1,722 \\
\hline Grand Total & 172,204 & 150,639 & 322,843 & 94,697 & 417,540 \\
\hline
\end{tabular}




\section{APPENDIX F}

Author's summary of South Dakota Workers by Age Groups, Occupations, and Income Levels 2011 (continued)

\begin{tabular}{|c|c|c|c|c|c|}
\hline \multicolumn{6}{|c|}{ Workers by Age, Occupation, and Income Levels - 2011} \\
\hline Percent of All Workers & \multicolumn{3}{|c|}{ Household Level $(<\$ 41,860 / y r)$} & \multirow[b]{2}{*}{$\begin{array}{c}\text { Higher Wage } \\
\text { (At least } \\
\$ 41,860 / \mathbf{y r}) \\
\end{array}$} & \multirow[b]{2}{*}{ Grand Total } \\
\hline AGE GROUPS & $\underset{(<\$ 20,930 / y r)}{\text { Low Wage }}$ & $\begin{array}{c}\text { Between Low Wage \& } \\
\text { Household } \\
(\$ 20,930-\$ 41,859 / \mathbf{y r}) \\
\end{array}$ & Total & & \\
\hline $16-29$ & $21.68 \%$ & $8.78 \%$ & $30.46 \%$ & $1.79 \%$ & $32.25 \%$ \\
\hline Management & $0.66 \%$ & $0.52 \%$ & $1.18 \%$ & $0.19 \%$ & $1.37 \%$ \\
\hline Business \& Finance & $0.22 \%$ & $0.28 \%$ & $0.50 \%$ & $0.11 \%$ & $0.61 \%$ \\
\hline Computer/Math & $0.12 \%$ & $0.08 \%$ & $0.20 \%$ & $0.10 \%$ & $0.31 \% *$ \\
\hline Architecture/Engineering & $0.15 \%$ & $0.14 \%$ & $0.29 \%$ & $0.11 \%$ & $0.40 \%$ \\
\hline Sciences & $0.17 \%$ & $0.06 \%$ & $0.23 \%$ & $0.03 \%$ & $0.26 \%$ \\
\hline Community \& Social Services & $0.07 \%$ & $0.21 \%$ & $0.28 \%$ & $0.01 \%$ & $0.29 \%$ \\
\hline Legal & $0.02 \%$ & $0.05 \%$ & $0.06 \% *$ & $0.02 \%$ & $0.09 \% *$ \\
\hline Education & $0.66 \%$ & $0.54 \%$ & $1.20 \%$ & $0.02 \%$ & $1.22 \%$ \\
\hline Arts/Entertainment & $0.35 \%$ & $0.11 \%$ & $0.45 \% *$ & $0.01 \%$ & $0.47 \% *$ \\
\hline Healthcare Practitioners & $0.41 \%$ & $0.44 \%$ & $0.85 \%$ & $0.29 \%$ & $1.13 \% *$ \\
\hline Healthcare Support & $0.91 \%$ & $0.28 \%$ & $1.19 \%$ & $0.00 \%$ & $1.19 \%$ \\
\hline Protective Services & $0.41 \%$ & $0.23 \%$ & $0.64 \%$ & $0.08 \%$ & $0.72 \%$ \\
\hline Food Services & $3.94 \%$ & $0.24 \%$ & $4.18 \%$ & $0.02 \%$ & $4.21 \% *$ \\
\hline Building/Grounds & $0.99 \%$ & $0.09 \%$ & $1.09 \% *$ & $0.02 \%$ & $1.10 \% *$ \\
\hline Personal Care & $1.27 \%$ & $0.22 \%$ & $1.48 \% *$ & $0.02 \%$ & $1.51 \% *$ \\
\hline Sales & $3.01 \%$ & $0.69 \%$ & $3.70 \%$ & $0.19 \%$ & $3.89 \%$ \\
\hline Office/Admin & $2.63 \%$ & $1.48 \%$ & $4.11 \%$ & $0.17 \%$ & $4.28 \%$ \\
\hline Farming/Fishing & $1.32 \%$ & $0.30 \%$ & $1.61 \% *$ & $0.02 \%$ & $1.63 \%$ \\
\hline Construction & $1.22 \%$ & $0.72 \%$ & $1.94 \%$ & $0.11 \%$ & $2.04 \% *$ \\
\hline Install/Maintenance & $0.52 \%$ & $0.61 \%$ & $1.14 \% *$ & $0.08 \%$ & $1.21 \% *$ \\
\hline Production & $1.17 \%$ & $1.12 \%$ & $2.29 \%$ & $0.11 \%$ & $2.40 \%$ \\
\hline Transportation & $1.47 \%$ & $0.38 \%$ & $1.84 \% *$ & $0.08 \%$ & $1.93 \% *$ \\
\hline 30-39 & $4.96 \%$ & $8.28 \%$ & $13.24 \%$ & $5.16 \%$ & $18.41 \%$ \\
\hline Management & $0.28 \%$ & $0.72 \%$ & $0.99 \% *$ & $1.01 \%$ & $2.00 \%$ \\
\hline Business \& Finance & $0.09 \%$ & $0.39 \%$ & $0.48 \%$ & $0.39 \%$ & $0.87 \%$ \\
\hline Computer/Math & $0.03 \%$ & $0.12 \%$ & $0.14 \% *$ & $0.29 \%$ & $0.43 \%$ \\
\hline Architecture/Engineering & $0.04 \%$ & $0.08 \%$ & $0.12 \%$ & $0.11 \%$ & $0.23 \%$ \\
\hline Sciences & $0.02 \%$ & $0.10 \%$ & $0.12 \%$ & $0.13 \%$ & $0.25 \%$ \\
\hline Community \& Social Services & $0.07 \%$ & $0.27 \%$ & $0.34 \%$ & $0.10 \%$ & $0.44 \%$ \\
\hline Legal & $0.03 \%$ & $0.09 \%$ & $0.12 \%$ & $0.07 \%$ & $0.18 \% *$ \\
\hline Education & $0.26 \%$ & $0.62 \%$ & $0.87 \% *$ & $0.11 \%$ & $0.98 \%$ \\
\hline Arts/Entertainment & $0.13 \%$ & $0.19 \%$ & $0.32 \%$ & $0.04 \%$ & $0.36 \%$ \\
\hline Healthcare Practitioners & $0.14 \%$ & $0.46 \%$ & $0.60 \%$ & $0.64 \%$ & $1.25 \% *$ \\
\hline Healthcare Support & $0.24 \%$ & $0.18 \%$ & $0.42 \%$ & $0.01 \%$ & $0.43 \%$ \\
\hline Protective Services & $0.09 \%$ & $0.19 \%$ & $0.28 \%$ & $0.13 \%$ & $0.41 \%$ \\
\hline Food Services & $0.39 \%$ & $0.14 \%$ & $0.52 \% *$ & $0.01 \%$ & $0.54 \% *$ \\
\hline Building/Grounds & $0.38 \%$ & $0.06 \%$ & $0.44 \%$ & $0.01 \%$ & $0.46 \% *$ \\
\hline
\end{tabular}

(continued)

DOI: $10.17605 / O S F . I O / Q F 4 H Y$ 


\section{APPENDIX F}

Author's summary of South Dakota Workers by Age Groups, Occupations, and Income Levels 2011 (continued)

\begin{tabular}{|c|c|c|c|c|c|}
\hline \multicolumn{6}{|c|}{ Workers by Age, Occupation, and Income Levels - 2011} \\
\hline Percent of All Workers & \multicolumn{3}{|c|}{ Household Level $(<\$ 41,860 / y r)$} & \multirow[b]{2}{*}{$\begin{array}{l}\text { Higher Wage } \\
\text { (At least } \\
\$ 41,860 / y r) \\
\end{array}$} & \multirow[b]{2}{*}{ Grand Total } \\
\hline AGE GROUPS & $\begin{array}{c}\text { Low Wage } \\
(<\$ 20,930 / y r)\end{array}$ & $\begin{array}{c}\text { Between Low Wage \& } \\
\text { Household } \\
(\$ 20,930-\$ 41,859 / y r) \\
\end{array}$ & Total & & \\
\hline \multicolumn{6}{|l|}{ 30-39 (continued) } \\
\hline Personal Care & $0.35 \%$ & $0.19 \%$ & $0.55 \% *$ & $0.07 \%$ & $0.62 \%$ \\
\hline Sales & $0.59 \%$ & $0.54 \%$ & $1.12 \% *$ & $0.63 \%$ & $1.75 \%$ \\
\hline Office/Admin & $0.85 \%$ & $1.32 \%$ & $2.17 \%$ & $0.17 \%$ & $2.33 \% *$ \\
\hline Farming/Fishing & $0.12 \%$ & $0.18 \%$ & $0.30 \%$ & $0.03 \%$ & $0.33 \%$ \\
\hline Construction & $0.23 \%$ & $0.63 \%$ & $0.87 \% *$ & $0.34 \%$ & $1.21 \%$ \\
\hline Install/Maintenance & $0.16 \%$ & $0.35 \%$ & $0.51 \%$ & $0.35 \%$ & $0.86 \%$ \\
\hline Production & $0.26 \%$ & $0.91 \%$ & $1.17 \%$ & $0.28 \%$ & $1.44 \% *$ \\
\hline Transportation & $0.23 \%$ & $0.56 \%$ & $0.79 \%$ & $0.25 \%$ & $1.04 \%$ \\
\hline $40-49$ & $5.08 \%$ & $8.92 \%$ & $14.00 \%$ & $6.46 \%$ & $20.46 \%$ \\
\hline Management & $0.52 \%$ & $0.83 \%$ & $1.35 \%$ & $1.48 \%$ & $2.83 \%$ \\
\hline Business \& Finance & $0.05 \%$ & $0.41 \%$ & $0.46 \%$ & $0.35 \%$ & $0.81 \%$ \\
\hline Computer/Math & $0.01 \%$ & $0.05 \%$ & $0.06 \%$ & $0.18 \%$ & $0.24 \%$ \\
\hline Architecture/Engineering & $0.02 \%$ & $0.07 \%$ & $0.10 \% *$ & $0.17 \%$ & $0.27 \%$ \\
\hline Sciences & $0.01 \%$ & $0.04 \%$ & $0.04 \% *$ & $0.08 \%$ & $0.12 \%$ \\
\hline Community \& Social Services & $0.09 \%$ & $0.17 \%$ & $0.26 \%$ & $0.13 \%$ & $0.39 \%$ \\
\hline Legal & $0.02 \%$ & $0.06 \%$ & $0.08 \%$ & $0.08 \%$ & $0.15 \% *$ \\
\hline Education & $0.34 \%$ & $0.65 \%$ & $0.98 \% *$ & $0.28 \%$ & $1.26 \%$ \\
\hline Arts/Entertainment & $0.09 \%$ & $0.08 \%$ & $0.18 \% *$ & $0.09 \%$ & $0.26 \% *$ \\
\hline Healthcare Practitioners & $0.09 \%$ & $0.41 \%$ & $0.50 \%$ & $0.78 \%$ & $1.28 \%$ \\
\hline Healthcare Support & $0.25 \%$ & $0.20 \%$ & $0.45 \%$ & $0.01 \%$ & $0.45 \% *$ \\
\hline Protective Services & $0.05 \%$ & $0.13 \%$ & $0.17 \% *$ & $0.18 \%$ & $0.36 \% *$ \\
\hline Food Services & $0.37 \%$ & $0.17 \%$ & $0.54 \%$ & $0.03 \%$ & $0.57 \%$ \\
\hline Building/Grounds & $0.40 \%$ & $0.21 \%$ & $0.61 \%$ & $0.02 \%$ & $0.62 \% *$ \\
\hline Personal Care & $0.24 \%$ & $0.24 \%$ & $0.48 \%$ & $0.06 \%$ & $0.54 \%$ \\
\hline Sales & $0.45 \%$ & $0.72 \%$ & $1.16 \% *$ & $0.67 \%$ & $1.83 \%$ \\
\hline Office/Admin & $0.91 \%$ & $2.12 \%$ & $3.02 \% *$ & $0.54 \%$ & $3.56 \%$ \\
\hline Farming/Fishing & $0.14 \%$ & $0.10 \%$ & $0.24 \%$ & $0.06 \%$ & $0.29 \% *$ \\
\hline Construction & $0.27 \%$ & $0.46 \%$ & $0.73 \%$ & $0.41 \%$ & $1.15 \% *$ \\
\hline Install/Maintenance & $0.15 \%$ & $0.36 \%$ & $0.51 \%$ & $0.28 \%$ & $0.79 \%$ \\
\hline Production & $0.36 \%$ & $0.98 \%$ & $1.35 \% *$ & $0.27 \%$ & $1.62 \%$ \\
\hline Transportation & $0.27 \%$ & $0.46 \%$ & $0.74 \% *$ & $0.33 \%$ & $1.06 \% *$ \\
\hline
\end{tabular}

(continued) 


\section{APPENDIX F}

Author's summary of South Dakota Workers by Age Groups, Occupations, and Income Levels 2011 (continued)

\begin{tabular}{|c|c|c|c|c|c|}
\hline \multicolumn{6}{|c|}{ Workers by Age, Occupation, and Income Levels - 2011} \\
\hline Percent of All Workers & \multicolumn{3}{|c|}{ Household Level $(<\$ 41,860 / y r)$} & \multirow[b]{2}{*}{$\begin{array}{l}\text { Higher Wage (At } \\
\text { least } \$ 41,860 / \mathbf{y r} \text { ) }\end{array}$} & \multirow[b]{2}{*}{ Grand Total } \\
\hline AGE GROUPS & $\underset{(<\$ 20,930 / y r)}{\text { Low Wage }}$ & $\begin{array}{cc}\text { Between Low Wage \& } \\
\text { Household } \\
\$ \$ \mathbf{2 0 0 , 9 3 0}- \\
\$ 41,859 / \mathrm{yr})\end{array}$ & Total & & \\
\hline $50-64$ & $7.06 \%$ & $9.85 \%$ & $16.91 \%$ & $7.43 \%$ & $24.34 \%$ \\
\hline Management & $0.82 \%$ & $1.01 \%$ & $1.83 \%$ & $1.85 \%$ & $3.69 \% *$ \\
\hline Business \& Finance & $0.09 \%$ & $0.36 \%$ & $0.45 \%$ & $0.37 \%$ & $0.82 \%$ \\
\hline Computer/Math & $0.06 \%$ & $0.04 \%$ & $0.10 \%$ & $0.15 \%$ & $0.25 \%$ \\
\hline Architecture/Engineering & $0.05 \%$ & $0.08 \%$ & $0.13 \%$ & $0.16 \%$ & $0.29 \%$ \\
\hline Sciences & $0.02 \%$ & $0.02 \%$ & $0.04 \%$ & $0.06 \%$ & $0.10 \%$ \\
\hline Community \& Social Services & $0.07 \%$ & $0.28 \%$ & $0.35 \%$ & $0.21 \%$ & $0.56 \%$ \\
\hline Legal & $0.01 \%$ & $0.04 \%$ & $0.05 \%$ & $0.12 \%$ & $0.17 \%$ \\
\hline Education & $0.44 \%$ & $0.87 \%$ & $1.31 \%$ & $0.66 \%$ & $1.97 \%$ \\
\hline Arts/Entertainment & $0.10 \%$ & $0.08 \%$ & $0.18 \%$ & $0.05 \%$ & $0.22 \% *$ \\
\hline Healthcare Practitioners & $0.14 \%$ & $0.53 \%$ & $0.67 \%$ & $0.83 \%$ & $1.50 \%$ \\
\hline Healthcare Support & $0.30 \%$ & $0.31 \%$ & $0.61 \%$ & $0.06 \%$ & $0.67 \%$ \\
\hline Protective Services & $0.07 \%$ & $0.12 \%$ & $0.19 \%$ & $0.06 \%$ & $0.26 \% *$ \\
\hline Food Services & $0.55 \%$ & $0.08 \%$ & $0.63 \%$ & $0.01 \%$ & $0.64 \%$ \\
\hline Building/Grounds & $0.47 \%$ & $0.45 \%$ & $0.92 \%$ & $0.08 \%$ & $1.00 \%$ \\
\hline Personal Care & $0.44 \%$ & $0.15 \%$ & $0.58 \% *$ & $0.02 \%$ & $0.60 \%$ \\
\hline Sales & $0.75 \%$ & $0.81 \%$ & $1.56 \%$ & $0.80 \%$ & $2.36 \%$ \\
\hline Office/Admin & $1.39 \%$ & $2.21 \%$ & $3.60 \%$ & $0.57 \%$ & $4.17 \%$ \\
\hline Farming/Fishing & $0.13 \%$ & $0.15 \%$ & $0.28 \%$ & $0.09 \%$ & $0.37 \%$ \\
\hline Construction & $0.21 \%$ & $0.48 \%$ & $0.69 \%$ & $0.40 \%$ & $1.09 \%$ \\
\hline Install/Maintenance & $0.14 \%$ & $0.28 \%$ & $0.43 \% *$ & $0.25 \%$ & $0.68 \%$ \\
\hline Production & $0.45 \%$ & $0.76 \%$ & $1.21 \%$ & $0.17 \%$ & $1.38 \%$ \\
\hline Transportation & $0.34 \%$ & $0.74 \%$ & $1.08 \%$ & $0.45 \%$ & $1.53 \%$ \\
\hline $65-95$ & $2.95 \%$ & $0.98 \%$ & $3.93 \%$ & $0.61 \%$ & $4.54 \%$ \\
\hline Management & $0.45 \%$ & $0.20 \%$ & $0.64 \% *$ & $0.27 \%$ & $0.91 \%$ \\
\hline Business \& Finance & $0.05 \%$ & $0.04 \%$ & $0.09 \%$ & $0.03 \%$ & $0.12 \%$ \\
\hline Computer/Math & $0.00 \%$ & $0.01 \%$ & $0.01 \%$ & $0.01 \%$ & $0.02 \%$ \\
\hline Architecture/Engineering & $0.01 \%$ & $0.00 \%$ & $0.01 \%$ & $0.00 \%$ & $0.01 \%$ \\
\hline Sciences & $0.01 \%$ & $0.00 \%$ & $0.01 \%$ & $0.00 \%$ & $0.01 \%$ \\
\hline Community \& Social Services & $0.06 \%$ & $0.05 \%$ & $0.11 \%$ & $0.05 \%$ & $0.17 \% *$ \\
\hline Legal & $0.02 \%$ & $0.01 \%$ & $0.02 \% *$ & $0.02 \%$ & $0.04 \%$ \\
\hline Education & $0.15 \%$ & $0.01 \%$ & $0.16 \%$ & $0.02 \%$ & $0.18 \%$ \\
\hline Arts/Entertainment & $0.08 \%$ & $0.02 \%$ & $0.10 \%$ & $0.01 \%$ & $0.11 \%$ \\
\hline Healthcare Practitioners & $0.05 \%$ & $0.04 \%$ & $0.08 \% *$ & $0.05 \%$ & $0.14 \% *$ \\
\hline Healthcare Support & $0.04 \%$ & $0.02 \%$ & $0.06 \%$ & $0.00 \%$ & $0.06 \%$ \\
\hline Protective Services & $0.02 \%$ & $0.01 \%$ & $0.03 \%$ & $0.00 \%$ & $0.03 \%$ \\
\hline Food Services & $0.20 \%$ & $0.05 \%$ & $0.25 \%$ & $0.00 \%$ & $0.25 \%$ \\
\hline Building/Grounds & $0.27 \%$ & $0.03 \%$ & $0.30 \%$ & $0.00 \%$ & $0.30 \%$ \\
\hline
\end{tabular}

(continued)

DOI: $10.17605 / O S F . I O / Q F 4 H Y$ 


\section{APPENDIX F}

Author's summary of South Dakota Workers by Age Groups, Occupations, and Income Levels 2011 (continued)

\begin{tabular}{|c|c|c|c|c|c|}
\hline \multicolumn{6}{|c|}{ Workers by Age, Occupation, and Income Levels - 2011} \\
\hline Percent of All Workers & \multicolumn{3}{|c|}{ Household Level $(<\$ 41,860 / y r)$} & \multirow[b]{2}{*}{$\begin{array}{l}\text { Higher Wage (At } \\
\text { least } \$ 41,860 / y r)\end{array}$} & \multirow[b]{2}{*}{ Grand Total } \\
\hline AGE GROUPS & $\begin{array}{c}\text { Low Wage } \\
(<\$ 20,930 / y r)\end{array}$ & $\begin{array}{c}\text { Between Low Wage } \& \\
\text { Household }(\$ 20,930- \\
\$ 41,859 / y \mathbf{y})\end{array}$ & Total & & \\
\hline \multicolumn{6}{|l|}{ 65-94 (continued) } \\
\hline Personal Care & $0.15 \%$ & $0.01 \%$ & $0.16 \%$ & $0.00 \%$ & $0.17 \% *$ \\
\hline Sales & $0.35 \%$ & $0.12 \%$ & $0.47 \%$ & $0.06 \%$ & $0.52 \% *$ \\
\hline Office/Admin & $0.46 \%$ & $0.18 \%$ & $0.64 \%$ & $0.03 \%$ & $0.66 \% *$ \\
\hline Farming/Fishing & $0.08 \%$ & $0.02 \%$ & $0.10 \%$ & $0.00 \%$ & $0.11 \% *$ \\
\hline Construction & $0.07 \%$ & $0.02 \%$ & $0.09 \%$ & $0.01 \%$ & $0.11 \% *$ \\
\hline Install/Maintenance & $0.05 \%$ & $0.02 \%$ & $0.07 \%$ & $0.01 \%$ & $0.08 \%$ \\
\hline Production & $0.11 \%$ & $0.05 \%$ & $0.16 \%$ & $0.01 \%$ & $0.17 \%$ \\
\hline Transportation & $0.29 \%$ & $0.08 \%$ & $0.37 \%$ & $0.02 \%$ & $0.39 \%$ \\
\hline Grand Total & $41.74 \%$ & $36.81 \%$ & $\mathbf{7 8 . 5 5 \%}$ & $21.45 \%$ & $100.00 \%$ \\
\hline
\end{tabular}




\section{APPENDIX F}

Author's summary of South Dakota Workers by Age Groups, Occupations, and Income Levels 2016 (continued)

\begin{tabular}{|c|c|c|c|c|c|}
\hline \multicolumn{6}{|c|}{ Workers by Age, Occupation, and Income Levels - 2016} \\
\hline Percent of All Workers & \multicolumn{3}{|c|}{ Household Level $(<\$ 45,873 / \mathrm{yr})$} & \multirow[b]{2}{*}{$\begin{array}{l}\text { Higher Wage (At } \\
\text { least } \$ 45,873 / y r \text { ) }\end{array}$} & \multirow[b]{2}{*}{ Grand Total } \\
\hline AGE GROUPS & $\begin{array}{c}\text { Low Wage } \\
(<\$ 22,936 / y r)\end{array}$ & $\begin{array}{c}\begin{array}{c}\text { Between Low Wage \& } \\
\text { Household } \\
\$ \$ 22,936- \\
\$ 45,872 / \mathrm{yr})\end{array} \\
\end{array}$ & Total & & \\
\hline $16-29$ & $21.08 \%$ & $8.45 \%$ & $29.54 \% *$ & $2.19 \%$ & $31.73 \%$ \\
\hline Management & $0.39 \%$ & $0.67 \%$ & $1.06 \%$ & $0.17 \%$ & $1.24 \% *$ \\
\hline Business \& Finance & $0.14 \%$ & $0.25 \%$ & $0.39 \%$ & $0.03 \%$ & $0.42 \%$ \\
\hline Computer/Math & $0.13 \%$ & $0.13 \%$ & $0.26 \%$ & $0.09 \%$ & $0.35 \%$ \\
\hline Architecture/Engineering & $0.08 \%$ & $0.12 \%$ & $0.20 \%$ & $0.14 \%$ & $0.34 \%$ \\
\hline Sciences & $0.08 \%$ & $0.15 \%$ & $0.24 \% *$ & $0.03 \%$ & $0.27 \%$ \\
\hline Community \& Social Services & $0.11 \%$ & $0.20 \%$ & $0.30 \% *$ & $0.00 \%$ & $0.30 \%$ \\
\hline Legal & $0.05 \%$ & $0.01 \%$ & $0.06 \%$ & $0.02 \%$ & $0.09 \% *$ \\
\hline Education & $0.62 \%$ & $0.42 \%$ & $1.04 \%$ & $0.01 \%$ & $1.05 \%$ \\
\hline Arts/Entertainment & $0.28 \%$ & $0.08 \%$ & $0.37 \% *$ & $0.06 \%$ & $0.43 \%$ \\
\hline Healthcare Practitioners & $0.42 \%$ & $0.70 \%$ & $1.12 \%$ & $0.27 \%$ & $1.39 \%$ \\
\hline Healthcare Support & $0.83 \%$ & $0.21 \%$ & $1.04 \%$ & $0.04 \%$ & $1.08 \%$ \\
\hline Protective Services & $0.36 \%$ & $0.18 \%$ & $0.54 \%$ & $0.04 \%$ & $0.58 \%$ \\
\hline Food Services & $4.51 \%$ & $0.24 \%$ & $4.76 \% *$ & $0.02 \%$ & $4.78 \%$ \\
\hline Building/Grounds & $1.07 \%$ & $0.18 \%$ & $1.24 \% *$ & $0.03 \%$ & $1.27 \%$ \\
\hline Personal Care & $1.52 \%$ & $0.30 \%$ & $1.82 \%$ & $0.11 \%$ & $1.93 \%$ \\
\hline Sales & $3.09 \%$ & $0.53 \%$ & $3.61 \% *$ & $0.24 \%$ & $3.85 \%$ \\
\hline Office/Admin & $2.79 \%$ & $1.20 \%$ & $3.99 \%$ & $0.05 \%$ & $4.04 \%$ \\
\hline Farming/Fishing & $0.66 \%$ & $0.48 \%$ & $1.14 \%$ & $0.06 \%$ & $1.20 \%$ \\
\hline Construction & $1.23 \%$ & $0.74 \%$ & $1.97 \%$ & $0.26 \%$ & $2.23 \%$ \\
\hline Install/Maintenance & $0.36 \%$ & $0.49 \%$ & $0.85 \%$ & $0.15 \%$ & $1.00 \%$ \\
\hline Production & $1.13 \%$ & $0.68 \%$ & $1.81 \%$ & $0.19 \%$ & $2.00 \%$ \\
\hline Transportation & $1.22 \%$ & $0.49 \%$ & $1.71 \%$ & $0.17 \%$ & $1.88 \%$ \\
\hline 30-39 & $5.71 \%$ & $8.67 \%$ & $14.38 \%$ & $5.18 \%$ & $19.56 \%$ \\
\hline Management & $0.63 \%$ & $0.87 \%$ & $1.50 \%$ & $0.95 \%$ & $2.44 \% *$ \\
\hline Business \& Finance & $0.10 \%$ & $0.28 \%$ & $0.38 \%$ & $0.42 \%$ & $0.80 \%$ \\
\hline Computer/Math & $0.01 \%$ & $0.10 \%$ & $0.11 \%$ & $0.20 \%$ & $0.32 \% *$ \\
\hline Architecture/Engineering & $0.02 \%$ & $0.05 \%$ & $0.07 \%$ & $0.23 \%$ & $0.30 \%$ \\
\hline Sciences & $0.01 \%$ & $0.11 \%$ & $0.13 \% *$ & $0.08 \%$ & $0.20 \% *$ \\
\hline Community \& Social Services & $0.07 \%$ & $0.22 \%$ & $0.29 \%$ & $0.02 \%$ & $0.31 \%$ \\
\hline Legal & $0.01 \%$ & $0.04 \%$ & $0.04 \% *$ & $0.07 \%$ & $0.11 \%$ \\
\hline Education & $0.27 \%$ & $0.60 \%$ & $0.87 \%$ & $0.21 \%$ & $1.08 \%$ \\
\hline Arts/Entertainment & $0.13 \%$ & $0.16 \%$ & $0.29 \%$ & $0.05 \%$ & $0.35 \% *$ \\
\hline Healthcare Practitioners & $0.14 \%$ & $0.57 \%$ & $0.71 \%$ & $0.78 \%$ & $1.49 \%$ \\
\hline Healthcare Support & $0.37 \%$ & $0.17 \%$ & $0.54 \%$ & $0.02 \%$ & $0.56 \%$ \\
\hline Protective Services & $0.04 \%$ & $0.15 \%$ & $0.20 \% *$ & $0.10 \%$ & $0.30 \%$ \\
\hline Food Services & $0.72 \%$ & $0.14 \%$ & $0.86 \%$ & $0.00 \%$ & $0.86 \%$ \\
\hline Building/Grounds & $0.39 \%$ & $0.20 \%$ & $0.59 \%$ & $0.04 \%$ & $0.63 \%$ \\
\hline
\end{tabular}

(continued)

DOI: $10.17605 / O S F . I O / Q F 4 H Y$ 


\section{APPENDIX F}

Author's summary of South Dakota Workers by Age Groups, Occupations, and Income Levels 2016 (continued)

\begin{tabular}{|c|c|c|c|c|c|}
\hline \multicolumn{6}{|c|}{ Workers by Age, Occupation, and Income Levels - 2016} \\
\hline Percent of All Workers & \multicolumn{3}{|c|}{ Household Level $(<\$ 45,873 / y r)$} & \multirow[b]{2}{*}{$\begin{array}{l}\text { Higher Wage (At } \\
\text { least } \$ 45,873 / y r)\end{array}$} & \multirow[b]{2}{*}{ Grand Total } \\
\hline AGE GROUPS & $\begin{array}{c}\text { Low Wage } \\
(<\$ 22,936 / y r)\end{array}$ & $\begin{array}{c}\text { Between Low Wage \& } \\
\text { Household } \quad(\$ 22,936- \\
\$ 45,872 / \mathbf{y r}) \\
\end{array}$ & Total & & \\
\hline \multicolumn{6}{|l|}{ 30-39 (continued) } \\
\hline Personal Care & $0.33 \%$ & $0.24 \%$ & $0.57 \%$ & $0.07 \%$ & $0.64 \%$ \\
\hline Sales & $0.57 \%$ & $0.60 \%$ & $1.16 \% *$ & $0.58 \%$ & $1.74 \%$ \\
\hline Office/Admin & $0.77 \%$ & $1.57 \%$ & $2.34 \%$ & $0.31 \%$ & $2.65 \%$ \\
\hline Farming/Fishing & $0.12 \%$ & $0.20 \%$ & $0.32 \%$ & $0.07 \%$ & $0.39 \%$ \\
\hline Construction & $0.26 \%$ & $0.64 \%$ & $0.90 \%$ & $0.26 \%$ & $1.16 \%$ \\
\hline Install/Maintenance & $0.07 \%$ & $0.32 \%$ & $0.39 \%$ & $0.24 \%$ & $0.62 \% *$ \\
\hline Production & $0.41 \%$ & $0.97 \%$ & $1.38 \%$ & $0.14 \%$ & $1.53 \% *$ \\
\hline Transportation & $0.27 \%$ & $0.46 \%$ & $0.73 \%$ & $0.33 \%$ & $1.06 \%$ \\
\hline $40-49$ & $4.38 \%$ & $\mathbf{7 . 4 4 \%}$ & $11.83 \%$ & $6.15 \%$ & $17.98 \%$ \\
\hline Management & $0.48 \%$ & $0.65 \%$ & $1.12 \% *$ & $1.46 \%$ & $2.58 \%$ \\
\hline Business \& Finance & $0.04 \%$ & $0.27 \%$ & $0.31 \%$ & $0.36 \%$ & $0.67 \%$ \\
\hline Computer/Math & $0.00 \%$ & $0.07 \%$ & $0.07 \%$ & $0.19 \%$ & $0.26 \%$ \\
\hline Architecture/Engineering & $0.00 \%$ & $0.02 \%$ & $0.02 \%$ & $0.20 \%$ & $0.22 \%$ \\
\hline Sciences & $0.01 \%$ & $0.01 \%$ & $0.01 \% *$ & $0.08 \%$ & $0.09 \%$ \\
\hline Community \& Social Services & $0.07 \%$ & $0.14 \%$ & $0.22 \% *$ & $0.07 \%$ & $0.29 \%$ \\
\hline Legal & $0.00 \%$ & $0.05 \%$ & $0.05 \%$ & $0.07 \%$ & $0.12 \%$ \\
\hline Education & $0.24 \%$ & $0.48 \%$ & $0.73 \% *$ & $0.28 \%$ & $1.01 \%$ \\
\hline Arts/Entertainment & $0.05 \%$ & $0.07 \%$ & $0.12 \%$ & $0.07 \%$ & $0.19 \%$ \\
\hline Healthcare Practitioners & $0.12 \%$ & $0.41 \%$ & $0.53 \%$ & $0.66 \%$ & $1.19 \%$ \\
\hline Healthcare Support & $0.13 \%$ & $0.21 \%$ & $0.34 \%$ & $0.01 \%$ & $0.35 \%$ \\
\hline Protective Services & $0.02 \%$ & $0.10 \%$ & $0.12 \%$ & $0.15 \%$ & $0.27 \%$ \\
\hline Food Services & $0.42 \%$ & $0.14 \%$ & $0.56 \%$ & $0.01 \%$ & $0.57 \%$ \\
\hline Building/Grounds & $0.44 \%$ & $0.16 \%$ & $0.60 \%$ & $0.05 \%$ & $0.65 \%$ \\
\hline Personal Care & $0.24 \%$ & $0.28 \%$ & $0.52 \%$ & $0.06 \%$ & $0.58 \%$ \\
\hline Sales & $0.43 \%$ & $0.60 \%$ & $1.02 \% *$ & $0.76 \%$ & $1.78 \%$ \\
\hline Office/Admin & $0.66 \%$ & $1.55 \%$ & $2.22 \% *$ & $0.45 \%$ & $2.67 \%$ \\
\hline Farming/Fishing & $0.08 \%$ & $0.15 \%$ & $0.23 \%$ & $0.03 \%$ & $0.26 \%$ \\
\hline Construction & $0.20 \%$ & $0.48 \%$ & $0.68 \%$ & $0.28 \%$ & $0.97 \% *$ \\
\hline Install/Maintenance & $0.07 \%$ & $0.32 \%$ & $0.39 \%$ & $0.25 \%$ & $0.65 \% *$ \\
\hline Production & $0.37 \%$ & $0.73 \%$ & $1.10 \%$ & $0.26 \%$ & $1.37 \% *$ \\
\hline Transportation & $0.31 \%$ & $0.55 \%$ & $0.86 \%$ & $0.40 \%$ & $1.26 \%$ \\
\hline
\end{tabular}

(continued) 


\section{APPENDIX F}

Author's summary of South Dakota Workers by Age Groups, Occupations, and Income Levels 2016 (continued)

\begin{tabular}{|c|c|c|c|c|c|}
\hline \multicolumn{6}{|c|}{ Workers by Age, Occupation, and Income Levels - 2016} \\
\hline Percent of All Workers & \multicolumn{3}{|c|}{ Household Level $(<\$ 45,873 / \mathrm{yr})$} & \multirow[b]{2}{*}{$\begin{array}{l}\text { Higher Wage (At } \\
\text { least } \$ 45,873 / y r \text { ) }\end{array}$} & \multirow[b]{2}{*}{ Grand Total } \\
\hline AGE GROUPS & $\begin{array}{c}\text { Low Wage } \\
(<\$ 22,936 / y r)\end{array}$ & $\begin{array}{c}\text { Between Low Wage \& } \\
\text { Household } \quad(\$ 22,936- \\
\$ 45,872 / \mathbf{y r}) \\
\end{array}$ & Total & & \\
\hline $50-64$ & $7.11 \%$ & $10.23 \%$ & $17.33 \% *$ & $8.18 \%$ & $25.52 \% *$ \\
\hline Management & $0.67 \%$ & $0.93 \%$ & $1.60 \%$ & $2.09 \%$ & $3.69 \%$ \\
\hline Business \& Finance & $0.14 \%$ & $0.36 \%$ & $0.50 \%$ & $0.50 \%$ & $1.00 \%$ \\
\hline Computer/Math & $0.02 \%$ & $0.07 \%$ & $0.09 \%$ & $0.24 \%$ & $0.32 \% *$ \\
\hline Architecture/Engineering & $0.00 \%$ & $0.05 \%$ & $0.05 \%$ & $0.17 \%$ & $0.23 \% *$ \\
\hline Sciences & $0.02 \%$ & $0.02 \%$ & $0.04 \%$ & $0.11 \%$ & $0.16 \% *$ \\
\hline Community \& Social Services & $0.07 \%$ & $0.37 \%$ & $0.44 \%$ & $0.11 \%$ & $0.55 \%$ \\
\hline Legal & $0.05 \%$ & $0.05 \%$ & $0.10 \%$ & $0.14 \%$ & $0.24 \%$ \\
\hline Education & $0.38 \%$ & $0.62 \%$ & $1.00 \%$ & $0.49 \%$ & $1.48 \% *$ \\
\hline Arts/Entertainment & $0.15 \%$ & $0.10 \%$ & $0.25 \%$ & $0.08 \%$ & $0.33 \%$ \\
\hline Healthcare Practitioners & $0.17 \%$ & $0.54 \%$ & $0.71 \%$ & $0.83 \%$ & $1.54 \%$ \\
\hline Healthcare Support & $0.21 \%$ & $0.28 \%$ & $0.49 \%$ & $0.01 \%$ & $0.50 \%$ \\
\hline Protective Services & $0.09 \%$ & $0.18 \%$ & $0.27 \%$ & $0.08 \%$ & $0.35 \%$ \\
\hline Food Services & $0.57 \%$ & $0.12 \%$ & $0.70 \% *$ & $0.01 \%$ & $0.71 \%$ \\
\hline Building/Grounds & $0.73 \%$ & $0.33 \%$ & $1.06 \%$ & $0.06 \%$ & $1.12 \%$ \\
\hline Personal Care & $0.45 \%$ & $0.22 \%$ & $0.67 \%$ & $0.07 \%$ & $0.74 \%$ \\
\hline Sales & $0.68 \%$ & $0.89 \%$ & $1.56 \% *$ & $0.95 \%$ & $2.52 \% *$ \\
\hline Office/Admin & $1.27 \%$ & $2.26 \%$ & $3.53 \%$ & $0.62 \%$ & $4.15 \%$ \\
\hline Farming/Fishing & $0.13 \%$ & $0.18 \%$ & $0.31 \%$ & $0.11 \%$ & $0.42 \%$ \\
\hline Construction & $0.37 \%$ & $0.60 \%$ & $0.96 \% *$ & $0.42 \%$ & $1.38 \%$ \\
\hline Install/Maintenance & $0.07 \%$ & $0.38 \%$ & $0.45 \%$ & $0.27 \%$ & $0.73 \% *$ \\
\hline Production & $0.39 \%$ & $0.91 \%$ & $1.29 \% *$ & $0.30 \%$ & $1.60 \% *$ \\
\hline Transportation & $0.48 \%$ & $0.79 \%$ & $1.27 \%$ & $0.51 \%$ & $1.78 \%$ \\
\hline $65-95$ & $2.96 \%$ & $1.28 \%$ & $4.24 \%$ & $0.97 \%$ & $5.21 \%$ \\
\hline Management & $0.25 \%$ & $0.21 \%$ & $0.46 \%$ & $0.34 \%$ & $0.80 \%$ \\
\hline Business \& Finance & $0.04 \%$ & $0.03 \%$ & $0.07 \%$ & $0.09 \%$ & $0.15 \% *$ \\
\hline Computer/Math & $0.00 \%$ & $0.00 \%$ & $0.01 \% *$ & $0.01 \%$ & $0.02 \%$ \\
\hline Architecture/Engineering & $0.01 \%$ & $0.00 \%$ & $0.01 \%$ & $0.02 \%$ & $0.03 \%$ \\
\hline Sciences & $0.00 \%$ & $0.01 \%$ & $0.01 \%$ & $0.01 \%$ & $0.02 \%$ \\
\hline Community \& Social Services & $0.05 \%$ & $0.03 \%$ & $0.08 \%$ & $0.04 \%$ & $0.12 \%$ \\
\hline Legal & $0.00 \%$ & $0.01 \%$ & $0.01 \%$ & $0.02 \%$ & $0.03 \%$ \\
\hline Education & $0.37 \%$ & $0.06 \%$ & $0.43 \%$ & $0.05 \%$ & $0.48 \%$ \\
\hline Arts/Entertainment & $0.04 \%$ & $0.00 \%$ & $0.04 \%$ & $0.00 \%$ & $0.04 \%$ \\
\hline Healthcare Practitioners & $0.07 \%$ & $0.06 \%$ & $0.13 \%$ & $0.05 \%$ & $0.18 \%$ \\
\hline Healthcare Support & $0.09 \%$ & $0.02 \%$ & $0.11 \%$ & $0.00 \%$ & $0.11 \%$ \\
\hline Protective Services & $0.03 \%$ & $0.01 \%$ & $0.04 \%$ & $0.01 \%$ & $0.05 \%$ \\
\hline Food Services & $0.19 \%$ & $0.02 \%$ & $0.21 \%$ & $0.01 \%$ & $0.22 \%$ \\
\hline Building/Grounds & $0.20 \%$ & $0.10 \%$ & $0.29 \% *$ & $0.01 \%$ & $0.31 \% *$ \\
\hline
\end{tabular}

(continued)

DOI: $10.17605 / O S F . I O / Q F 4 H Y$ 


\section{APPENDIX F}

Author's summary of South Dakota Workers by Age Groups, Occupations, and Income Levels 2016 (continued)

\begin{tabular}{|c|c|c|c|c|c|}
\hline \multicolumn{6}{|c|}{ Workers by Age, Occupation, and Income Levels - 2016} \\
\hline Percent of All Workers & \multicolumn{3}{|c|}{ Household Level $(<\$ 45,873 / \mathrm{yr})$} & \multirow[b]{2}{*}{$\begin{array}{l}\text { Higher Wage (At } \\
\text { least } \$ 45,873 / y r \text { ) }\end{array}$} & \multirow[b]{2}{*}{ Grand Total } \\
\hline AGE GROUPS & $\begin{array}{c}\text { Low Wage } \\
(<\$ 22,936 / \mathbf{y r})\end{array}$ & $\begin{array}{cc}\text { Between Low Wage \& } \\
\text { Household } \\
\$ \$ 222,936- \\
\$ 45,872 / \mathbf{y r})\end{array}$ & Total & & \\
\hline \multicolumn{6}{|l|}{ 65-94 (continued) } \\
\hline Personal Care & $0.21 \%$ & $0.05 \%$ & $0.26 \%$ & $0.01 \%$ & $0.28 \% *$ \\
\hline Sales & $0.42 \%$ & $0.10 \%$ & $0.52 \%$ & $0.14 \%$ & $0.65 \% *$ \\
\hline Office/Admin & $0.42 \%$ & $0.25 \%$ & $0.67 \%$ & $0.04 \%$ & $0.71 \%$ \\
\hline Farming/Fishing & $0.05 \%$ & $0.02 \%$ & $0.06 \% *$ & $0.01 \%$ & $0.08 \% *$ \\
\hline Construction & $0.06 \%$ & $0.04 \%$ & $0.10 \%$ & $0.04 \%$ & $0.14 \%$ \\
\hline Install/Maintenance & $0.10 \%$ & $0.05 \%$ & $0.15 \%$ & $0.02 \%$ & $0.17 \%$ \\
\hline Production & $0.11 \%$ & $0.11 \%$ & $0.22 \%$ & $0.01 \%$ & $0.23 \%$ \\
\hline Transportation & $0.27 \%$ & $0.10 \%$ & $0.37 \%$ & $0.05 \%$ & $0.41 \% *$ \\
\hline Grand Total & $41.24 \%$ & $36.08 \%$ & $77.32 \%$ & $22.68 \%$ & $100.00 \%$ \\
\hline
\end{tabular}

Note: * refers to statistical inconsistencies due to rounding.

Source: Ruggles et al. (2015). Integrated Public Use Microdata Series: Version 6.0 [dataset]. Minneapolis:

University of Minnesota. doi:10.18128/D010.V6.0 


\section{APPENDIX G}

\section{Family Economic Self-Sufficiency Weighted Average Summaries for 38 States}

\begin{tabular}{|c|c|c|c|c|c|}
\hline \multicolumn{3}{|c|}{ ALABAMA 2006-2010 ACS DATA } & \multicolumn{3}{|c|}{ ALABAMA 2011-2015 ACS DATA } \\
\hline Labor force & $3,372,243$ & & Labor force & $3,388,448$ & \\
\hline Single earner below $\$ 9.83 / \mathrm{hr}(\$ 20,761)$ & $1,421,821$ & $42.16 \%$ & $\begin{array}{l}\text { Single earner below } \$ 10.62 / \mathrm{hr} \\
(\$ 22,429)\end{array}$ & $1,480,237$ & $43.68 \%$ \\
\hline $\begin{array}{l}\text { Household low wage threshold } \\
(\$ 41,522)\end{array}$ & $2,464,869$ & $73.09 \%$ & $\begin{array}{l}\text { Household low wage threshold } \\
(\$ 44,859)\end{array}$ & $2,473,269$ & $72.99 \%$ \\
\hline \multicolumn{3}{|c|}{ ARIZONA 2006-2010 ACS DATA } & \multicolumn{3}{|c|}{ ARIZONA 2011-2015 ACS DATA } \\
\hline Labor force & $4,699,965$ & & Labor force & $4,964,385$ & \\
\hline $\begin{array}{l}\text { Single earner below } \$ 12.78 / \mathrm{hr} \\
(\$ 26,992)\end{array}$ & $2,326,833$ & $49.51 \%$ & $\begin{array}{l}\text { Single earner below } \$ 13.94 / \mathrm{hr} \\
(\$ 29,442)\end{array}$ & $2,604,804$ & $52.47 \%$ \\
\hline $\begin{array}{l}\text { Household low wage threshold } \\
(\$ 69,189)\end{array}$ & $3,795,015$ & $80.75 \%$ & $\begin{array}{l}\text { Household low wage threshold } \\
(\$ 58,883)\end{array}$ & $4,076,167$ & $82.11 \%$ \\
\hline \multicolumn{3}{|c|}{ CALIFORNIA 2006-2010 ACS DATA } & \multicolumn{3}{|c|}{ CALIFORNIA 2011-2015 ACS DATA } \\
\hline Labor force & $31,466,807$ & & Labor force & $33,559,832$ & \\
\hline $\begin{array}{l}\text { Single earner below } \$ 16.38 / \mathrm{hr} \\
(\$ 34,595)\end{array}$ & $18,565,724$ & $59.00 \%$ & $\begin{array}{l}\text { Single earner below } \$ 16.82 / \mathrm{hr} \\
(\$ 35,524)\end{array}$ & $20,170,957$ & $60.10 \%$ \\
\hline $\begin{array}{l}\text { Household low wage threshold } \\
(\$ 53,983)\end{array}$ & $26,469,738$ & $84.12 \%$ & $\begin{array}{l}\text { Household low wage threshold } \\
(\$ 71,048)\end{array}$ & $28,026,482$ & $83.51 \%$ \\
\hline \multicolumn{3}{|c|}{ COLORADO 2006-2010 ACS DATA } & \multicolumn{3}{|c|}{ COLORADO 2011-2015 ACS DATA } \\
\hline Labor force & $4,034,703$ & & Labor force & $4,478,900$ & \\
\hline $\begin{array}{l}\text { Single earner below } \$ 13.74 / \mathrm{hr} \\
(\$ 29,019)\end{array}$ & $1,981,626$ & $49.11 \%$ & $\begin{array}{l}\text { Single earner below } \$ 15.49 / \mathrm{hr} \\
(\$ 32,715)\end{array}$ & $2,378,485$ & $53.10 \%$ \\
\hline $\begin{array}{l}\text { Household low wage threshold } \\
(\$ 58,038)\end{array}$ & $3,203,259$ & $79.39 \%$ & $\begin{array}{l}\text { Household low wage threshold } \\
(\$ 65,430)\end{array}$ & $3,653,551$ & $81.57 \%$ \\
\hline \multicolumn{3}{|c|}{ CONNECTICUT 2006-2010 ACS DATA } & \multicolumn{3}{|c|}{ CONNECTICUT 2011-2015 ACS DATA } \\
\hline Labor force & $2,965,949$ & & Labor force & $3,091,828$ & \\
\hline $\begin{array}{l}\text { Single earner below } \$ 15.95 / \mathrm{hr} \\
(\$ 33,687)\end{array}$ & $1,462,679$ & $49.32 \%$ & $\begin{array}{l}\text { Single earner below } \$ 25.21 / \mathrm{hr} \\
(\$ 53,244)\end{array}$ & $2,102,627$ & $68.01 \%$ \\
\hline $\begin{array}{l}\text { Household low wage threshold } \\
(\$ 67,373)\end{array}$ & $2,354,532$ & $79.39 \%$ & $\begin{array}{l}\text { Household low wage threshold } \\
(\$ 106,487)\end{array}$ & $2,810,609$ & $90.90 \%$ \\
\hline \multicolumn{3}{|c|}{ DELAWARE 2006-2010 ACS DATA } & \multicolumn{3}{|c|}{ DELAWARE 2011-2015 ACS DATA } \\
\hline Labor force & 725,045 & & Labor force & 767,317 & \\
\hline $\begin{array}{l}\text { Single earner below } \$ 11.36 / \mathrm{hr} \\
(\$ 23,993)\end{array}$ & 292,043 & $40.28 \%$ & $\begin{array}{l}\text { Single earner below } \$ 12.18 / \mathrm{hr} \\
(\$ 25,724)\end{array}$ & 327,189 & $42.64 \%$ \\
\hline $\begin{array}{l}\text { Household low wage threshold } \\
(\$ 47,985)\end{array}$ & 524,846 & $72.39 \%$ & $\begin{array}{l}\text { Household low wage threshold } \\
(\$ 51,448)\end{array}$ & 560,556 & $73.05 \%$ \\
\hline \multicolumn{3}{|c|}{ FLORIDA 2006-2010 ACS DATA } & \multicolumn{3}{|c|}{ FLORIDA 2011-2015 ACS DATA } \\
\hline Labor force & $14,355,083$ & & Labor force & $15,306,282$ & \\
\hline $\begin{array}{l}\text { Single earner below } \$ 12.26 / \mathrm{hr} \\
(\$ 25,893)\end{array}$ & $7,176,382$ & $49.99 \%$ & $\begin{array}{l}\text { Single earner below } \$ 13.19 / \mathrm{hr} \\
(\$ 27,858)\end{array}$ & $8,210,582$ & $53.64 \%$ \\
\hline $\begin{array}{l}\text { Household low wage threshold } \\
(\$ 51,786)\end{array}$ & $11,719,882$ & $81.64 \%$ & $\begin{array}{l}\text { Household low wage threshold } \\
(\$ 55,715)\end{array}$ & $12,739,198$ & $83.23 \%$ \\
\hline \multicolumn{3}{|c|}{ GEORGIA 2006-2010 ACS DATA } & \multicolumn{3}{|c|}{ GEORGIA 2011-2015 ACS DATA } \\
\hline Labor force & $7,235,749$ & & Labor force & $7,576,659$ & \\
\hline $\begin{array}{l}\text { Single earner below } \$ 10.81 / \mathrm{hr} \\
(\$ 22,831)\end{array}$ & $3,093,647$ & $42.76 \%$ & $\begin{array}{l}\text { Single earner below } \$ 11.69 / \mathrm{hr} \\
(\$ 24,690)\end{array}$ & $3,442,855$ & $45.44 \%$ \\
\hline $\begin{array}{l}\text { Household low wage threshold } \\
(\$ 45,661)\end{array}$ & $5,357,630$ & $74.04 \%$ & $\begin{array}{l}\text { Household low wage threshold } \\
(\$ 49,379)\end{array}$ & $5,700,198$ & $75.23 \%$ \\
\hline \multicolumn{3}{|c|}{ HAWAII 2006-2010 ACS DATA } & \multicolumn{3}{|c|}{ HAWAII 2011-2015 ACS DATA } \\
\hline Labor force & $1,394,885$ & & Labor force & $1,486,906$ & \\
\hline $\begin{array}{l}\text { Single earner below } \$ 13.47 / \mathrm{hr} \\
(\$ 28,449)\end{array}$ & 672,399 & $48.20 \%$ & $\begin{array}{l}\text { Single earner below } \$ 14.51 / \mathrm{hr} \\
(\$ 30,645)\end{array}$ & 754,986 & $50.78 \%$ \\
\hline $\begin{array}{l}\text { Household low wage threshold } \\
(\$ 56,897)\end{array}$ & $1,156,337$ & $82.90 \%$ & $\begin{array}{l}\text { Household low wage threshold } \\
(\$ 61,290)\end{array}$ & $1,234,971$ & $83.06 \%$ \\
\hline
\end{tabular}

(continued) 


\section{APPENDIX G}

Family Economic Self-Sufficiency Weighted Average Summaries for 38 States (continued)

\begin{tabular}{|c|c|c|c|c|c|}
\hline \multicolumn{3}{|c|}{ ILLINOIS 2006-2010 ACS DATA } & \multicolumn{3}{|c|}{ ILLINOIS 2011-2015 ACS DATA } \\
\hline Labor force & $10,387,342$ & & Labor force & $10,633,297$ & \\
\hline $\begin{array}{l}\text { Single earner below } \$ 13.51 / \mathrm{hr} \\
(\$ 28,533)\end{array}$ & $5,207,045$ & $50.13 \%$ & $\begin{array}{l}\text { Single earner below } \$ 14.55 / \mathrm{hr} \\
(\$ 30,730)\end{array}$ & $5,529,805$ & $52.00 \%$ \\
\hline $\begin{array}{l}\text { Household low wage threshold } \\
(\$ 57,066)\end{array}$ & $8,262,734$ & $79.55 \%$ & $\begin{array}{l}\text { Household low wage threshold } \\
(\$ 61,459)\end{array}$ & $8,472,202$ & $79.68 \%$ \\
\hline \multicolumn{3}{|c|}{ INDIANA 2006-2010 ACS DATA } & \multicolumn{3}{|c|}{ INDIANA 2011-2015 ACS DATA } \\
\hline Labor force & $4,921,614$ & & Labor force & $5,076,409$ & \\
\hline $\begin{array}{l}\text { Single earner below } \$ 10.84 / \mathrm{hr} \\
(\$ 22,894)\end{array}$ & $2,166,973$ & $44.03 \%$ & $\begin{array}{l}\text { Single earner below } \$ 12.54 / \mathrm{hr} \\
(\$ 26,485)\end{array}$ & $2,502,039$ & $49.29 \%$ \\
\hline $\begin{array}{l}\text { Household low wage threshold } \\
(\$ 45,788)\end{array}$ & $3,760,113$ & $76.40 \%$ & $\begin{array}{l}\text { Household low wage threshold } \\
(\$ 52,969)\end{array}$ & $4,069,851$ & $80.17 \%$ \\
\hline \multicolumn{3}{|c|}{ KENTUCKY 2006-2010 ACS DATA } & \multicolumn{3}{|c|}{ KENTUCKY 2011-2015 ACS DATA } \\
\hline Labor force & $3,091,839$ & & Labor force & $3,175,454$ & \\
\hline $\begin{array}{l}\text { Single earner below } \$ 11.30 / \mathrm{hr} \\
(\$ 23,866)\end{array}$ & $1,473,756$ & $47.67 \%$ & $\begin{array}{l}\text { Single earner below } \$ 12.04 / \mathrm{hr} \\
(\$ 25,429)\end{array}$ & $1,543,279$ & $48.60 \%$ \\
\hline $\begin{array}{l}\text { Household low wage threshold } \\
(\$ 47,731)\end{array}$ & $2,456,429$ & $79.45 \%$ & $\begin{array}{l}\text { Household low wage threshold } \\
(\$ 50,857)\end{array}$ & $2,509,092$ & $79.02 \%$ \\
\hline \multicolumn{3}{|c|}{ LOUISIANA 2006-2010 ACS DATA } & \multicolumn{3}{|c|}{ LOUISIANA 2011-2015 ACS DATA } \\
\hline Labor force & $3,339,915$ & & Labor force & $3,439,671$ & \\
\hline Single earner below $\$ 9.30 / \mathrm{hr}(\$ 19,663)$ & $1,309,858$ & $39.22 \%$ & Single earner below $\$ 9.80 / \mathrm{hr}(\$ 20,698)$ & $1,349,936$ & $39.25 \%$ \\
\hline $\begin{array}{l}\text { Household low wage threshold } \\
(\$ 39,325)\end{array}$ & $2,294,964$ & $68.71 \%$ & $\begin{array}{l}\text { Household low wage threshold } \\
(\$ 41,395)\end{array}$ & $2,332,515$ & $67.81 \%$ \\
\hline \multicolumn{3}{|c|}{ MARYLAND 2006-2010 ACS DATA } & \multicolumn{3}{|c|}{ MARYLAND 2010-2015 ACS DATA } \\
\hline Labor force & $5,049,789$ & & Labor force & $5,368,869$ & \\
\hline $\begin{array}{l}\text { Single earner below } \$ 15.42 / \mathrm{hr} \\
(\$ 32,567)\end{array}$ & $2,438,783$ & $48.29 \%$ & $\begin{array}{l}\text { Single earner below } \$ 17.54 / \mathrm{hr} \\
(\$ 37,045)\end{array}$ & $2,804,505$ & $52.24 \%$ \\
\hline $\begin{array}{l}\text { Household low wage threshold } \\
(\$ 65,134)\end{array}$ & $3,964,469$ & $78.51 \%$ & $\begin{array}{l}\text { Household low wage threshold } \\
(\$ 74,089)\end{array}$ & $4,302,867$ & $80.14 \%$ \\
\hline \multicolumn{3}{|c|}{ MASSACHUSETTS 2006-2010 ACS DATA } & \multicolumn{3}{|c|}{ MASSACHUSETTS 2011-2015 ACS DATA } \\
\hline Labor force & $5,558,946$ & & Labor force & $5,948,484$ & \\
\hline $\begin{array}{l}\text { Single earner below } \$ 15.93 / \mathrm{hr} \\
(\$ 33,644)\end{array}$ & $2,763,952$ & $49.72 \%$ & $\begin{array}{l}\text { Single earner below } \$ 17.15 / \mathrm{hr} \\
(\$ 36,221)\end{array}$ & $3,081,546$ & $51.80 \%$ \\
\hline $\begin{array}{l}\text { Household low wage threshold } \\
(\$ 67,288)\end{array}$ & $4,494,582$ & $80.85 \%$ & $\begin{array}{l}\text { Household low wage threshold } \\
(\$ 72,442)\end{array}$ & $4,765,665$ & $80.12 \%$ \\
\hline \multicolumn{3}{|c|}{ MISSISSIPPI 2006-2010 ACS DATA } & \multicolumn{3}{|c|}{ MISSISSIPPI 2011-2015 ACS DATA } \\
\hline Labor force & $2,051,924$ & & Labor force & $2,050,660$ & \\
\hline Single earner below $\$ 9.03 / \mathrm{hr}(\$ 19,072)$ & 822,894 & $40.10 \%$ & Single earner below $\$ 9.64 / \mathrm{hr}(\$ 20,360)$ & 841,997 & $41.06 \%$ \\
\hline $\begin{array}{l}\text { Household low wage threshold } \\
(\$ 38,143)\end{array}$ & $1,489,423$ & $72.59 \%$ & $\begin{array}{l}\text { Household low wage threshold } \\
(\$ 40,719)\end{array}$ & $1,508,825$ & $73.58 \%$ \\
\hline \multicolumn{3}{|c|}{ MISSOURI 2006-2010 ACS DATA } & \multicolumn{3}{|c|}{ MISSOURI 2011-2015 ACS DATA } \\
\hline Labor force & $4,564,815$ & & Labor force & $4,717,536$ & \\
\hline Single earner below $\$ 9.98 / \mathrm{hr}(\$ 21,078)$ & $1,899,542$ & $41.61 \%$ & $\begin{array}{l}\text { Single earner below } \$ 10.79 / \mathrm{hr} \\
(\$ 22,789)\end{array}$ & $2,027,292$ & $42.97 \%$ \\
\hline $\begin{array}{l}\text { Household low wage threshold } \\
(\$ 42,156)\end{array}$ & $3,350,450$ & $73.40 \%$ & $\begin{array}{l}\text { Household low wage threshold } \\
(\$ 45,577)\end{array}$ & $3,484,263$ & $73.86 \%$ \\
\hline \multicolumn{3}{|c|}{ MONTANA 2006-2010 ACS DATA } & \multicolumn{3}{|c|}{ MONTANA 2011-2015 ACS DATA } \\
\hline Labor force & 761,541 & & Labor force & 795,134 & \\
\hline $\begin{array}{l}\text { Single earner below } \$ 10.84 / \mathrm{hr} \\
(\$ 22,894)\end{array}$ & 365,218 & $47.96 \%$ & $\begin{array}{l}\text { Single earner below } \$ 11.68 / \mathrm{hr} \\
(\$ 24,668)\end{array}$ & 379,828 & $47.77 \%$ \\
\hline $\begin{array}{l}\text { Household low wage threshold } \\
(\$ 45,788)\end{array}$ & 607,438 & $79.76 \%$ & $\begin{array}{l}\text { Household low wage threshold } \\
(\$ 49,336)\end{array}$ & 617,918 & $77.71 \%$ \\
\hline
\end{tabular}

(continued) 


\section{APPENDIX G}

Family Economic Self-Sufficiency Weighted Average Summaries for 38 States (continued)

\begin{tabular}{|c|c|c|c|c|c|}
\hline \multicolumn{3}{|c|}{ NEBRASKA 2006-2010 ACS DATA } & \multicolumn{3}{|c|}{ NEBRASKA 2011-2015 ACS DATA } \\
\hline Labor force & $1,527,689$ & & Labor force & $1,597,705$ & \\
\hline $\begin{array}{l}\text { Single earner below } \$ 10.91 / \mathrm{hr} \\
(\$ 23,042)\end{array}$ & 696,198 & $45.57 \%$ & $\begin{array}{l}\text { Single earner below } \$ 11.66 / \mathrm{hr} \\
(\$ 24,626)\end{array}$ & 698,294 & $43.71 \%$ \\
\hline $\begin{array}{l}\text { Household low wage threshold } \\
(\$ 46,084)\end{array}$ & $1,210,848$ & $79.26 \%$ & $\begin{array}{l}\text { Household low wage threshold } \\
(\$ 49,252)\end{array}$ & $1,214,518$ & $76.02 \%$ \\
\hline \multicolumn{3}{|c|}{ NEVADA 2006-2010 ACS DATA } & \multicolumn{3}{|c|}{ NEVADA 2011-2015 ACS DATA } \\
\hline Labor force & $2,207,108$ & & Labor force & $2,290,971$ & \\
\hline $\begin{array}{l}\text { Single earner below } \$ 11.63 / \mathrm{hr} \\
(\$ 24,563)\end{array}$ & 899,166 & $40.74 \%$ & $\begin{array}{l}\text { Single earner below } \$ 12.52 / \mathrm{hr} \\
(\$ 26,442)\end{array}$ & $1,098,285$ & $47.94 \%$ \\
\hline $\begin{array}{l}\text { Household low wage threshold } \\
(\$ 49,125)\end{array}$ & $1,682,868$ & $76.25 \%$ & $\begin{array}{l}\text { Household low wage threshold } \\
(\$ 52,884)\end{array}$ & $1,844,381$ & $80.51 \%$ \\
\hline \multicolumn{3}{|c|}{ NEW JERSEY 2006-2010 ACS DATA } & \multicolumn{3}{|c|}{ NEW JERSEY 2011-2015 ACS DATA } \\
\hline Labor force & $7,488,276$ & & Labor force & $7,770,670$ & \\
\hline $\begin{array}{l}\text { Single earner below } \$ 16.30 / \mathrm{hr} \\
(\$ 34,426)\end{array}$ & $3,743,420$ & $49.99 \%$ & $\begin{array}{l}\text { Single earner below } \$ 17.54 / \mathrm{hr} \\
(\$ 37,045)\end{array}$ & $4,142,334$ & $53.31 \%$ \\
\hline $\begin{array}{l}\text { Household low wage threshold } \\
(\$ 68,851)\end{array}$ & $5,934,077$ & $79.24 \%$ & $\begin{array}{l}\text { Household low wage threshold } \\
(\$ 74,089)\end{array}$ & $6,199,288$ & $79.78 \%$ \\
\hline \multicolumn{3}{|c|}{ NEW YORK 2006-2010 ACS DATA } & \multicolumn{3}{|c|}{ NEW YORK 2011-2015 ACS DATA } \\
\hline Labor force & $15,919,727$ & & Labor force & $16,772,277$ & \\
\hline $\begin{array}{l}\text { Single earner below } \$ 15.88 / \mathrm{hr} \\
(\$ 33,539)\end{array}$ & $8,575,960$ & $53.87 \%$ & $\begin{array}{l}\text { Single earner below } \$ 17.10 / \mathrm{hr} \\
(\$ 36,115)\end{array}$ & $9,301,652$ & $55.46 \%$ \\
\hline $\begin{array}{l}\text { Household low wage threshold } \\
(\$ 67,077)\end{array}$ & $13,206,271$ & $82.96 \%$ & $\begin{array}{l}\text { Household low wage threshold } \\
(\$ 72,230)\end{array}$ & $13,835,250$ & $82.49 \%$ \\
\hline \multicolumn{3}{|c|}{ NORTH CAROLINA 2006-2010 ACS DATA } & \multicolumn{3}{|c|}{ NORTH CAROLINA 2011-2015 ACS DATA } \\
\hline Labor force & $6,986,893$ & & Labor force & $7,393,650$ & \\
\hline $\begin{array}{l}\text { Single earner below } \$ 10.01 / \mathrm{hr} \\
(\$ 21,141)\end{array}$ & $2,860,792$ & $40.95 \%$ & $\begin{array}{l}\text { Single earner below } \$ 13.58 / \mathrm{hr} \\
(\$ 28,681)\end{array}$ & $3,899,009$ & $52.73 \%$ \\
\hline $\begin{array}{l}\text { Household low wage threshold } \\
(\$ 42,282)\end{array}$ & $5,146,867$ & $73.66 \%$ & $\begin{array}{l}\text { Household low wage threshold } \\
(\$ 57,362)\end{array}$ & $6,138,678$ & $83.03 \%$ \\
\hline \multicolumn{3}{|c|}{ OHIO 2006-2010 ACS DATA } & \multicolumn{3}{|c|}{ OHIO 2011-2015 ACS DATA } \\
\hline Labor force & $8,788,559$ & & Labor force & $8,955,224$ & \\
\hline $\begin{array}{l}\text { Single earner below } \$ 11.79 / \mathrm{hr} \\
(\$ 24,901)\end{array}$ & $4,107,431$ & $46.74 \%$ & $\begin{array}{l}\text { Single earner below } \$ 12.93 / \mathrm{hr} \\
(\$ 27,308)\end{array}$ & $4,403,145$ & $49.17 \%$ \\
\hline $\begin{array}{l}\text { Household low wage threshold } \\
(\$ 49,801)\end{array}$ & $6,830,389$ & $77.72 \%$ & $\begin{array}{l}\text { Household low wage threshold } \\
(\$ 54,616)\end{array}$ & $7,084,357$ & $79.11 \%$ \\
\hline \multicolumn{3}{|c|}{ OKLAHOMA 2006-2010 ACS DATA } & \multicolumn{3}{|c|}{ OKLAHOMA 2011-2015 ACS DATA } \\
\hline Labor force & $2,780,228$ & & Labor force & $2,919,793$ & \\
\hline $\begin{array}{l}\text { Single earner below } \$ 10.04 / \mathrm{hr} \\
(\$ 21,205)\end{array}$ & $1,251,063$ & $45.00 \%$ & $\begin{array}{l}\text { Single earner below } \$ 10.83 / \mathrm{hr} \\
(\$ 22,873)\end{array}$ & $1,258,883$ & $43.12 \%$ \\
\hline $\begin{array}{l}\text { Household low wage threshold } \\
(\$ 42,409)\end{array}$ & $2,120,169$ & $76.26 \%$ & $\begin{array}{l}\text { Household low wage threshold } \\
(\$ 45,746)\end{array}$ & $2,190,306$ & $75.02 \%$ \\
\hline \multicolumn{3}{|c|}{ OREGON 2006-2010 ACS DATA } & \multicolumn{3}{|c|}{ OREGON 2011-2015 ACS DATA } \\
\hline Labor force & $2,928,935$ & & Labor force & $3,073,393$ & \\
\hline $\begin{array}{l}\text { Single earner below } \$ 11.47 / \mathrm{hr} \\
(\$ 24,225)\end{array}$ & $1,371,527$ & $46.83 \%$ & $\begin{array}{l}\text { Single earner below } \$ 13.36 / \mathrm{hr} \\
(\$ 28,217)\end{array}$ & $1,612,372$ & $52.46 \%$ \\
\hline $\begin{array}{l}\text { Household low wage threshold } \\
(\$ 48,449)\end{array}$ & $2,245,982$ & $76.68 \%$ & $\begin{array}{l}\text { Household low wage threshold } \\
(\$ 56,433)\end{array}$ & $2,469,934$ & $80.37 \%$ \\
\hline \multicolumn{3}{|c|}{ PENNSYLVANIA 2006-2010 ACS DATA } & \multicolumn{3}{|c|}{ PENNSYLVANIA 2011-2015 ACS DATA } \\
\hline Labor force & $9,918,850$ & & Labor force & $10,217,142$ & \\
\hline $\begin{array}{l}\text { Single earner below } \$ 13.46 / \mathrm{hr} \\
(\$ 28,428)\end{array}$ & $5,015,402$ & $50.56 \%$ & $\begin{array}{l}\text { Single earner below } \$ 14.20 / \mathrm{hr} \\
(\$ 29,991)\end{array}$ & $5,029,855$ & $49.23 \%$ \\
\hline $\begin{array}{l}\text { Household low wage threshold } \\
(\$ 56,855)\end{array}$ & $8,137,190$ & $82.04 \%$ & $\begin{array}{l}\text { Household low wage threshold } \\
(\$ 59,981)\end{array}$ & $8,185,789$ & $80.12 \%$ \\
\hline
\end{tabular}

(continued) 


\section{APPENDIX G}

Family Economic Self-Sufficiency Weighted Average Summaries for 38 States (continued)

\begin{tabular}{|c|c|c|c|c|c|}
\hline \multicolumn{3}{|c|}{ SOUTH CAROLINA 2006-2010 ACS DATA } & \multicolumn{3}{|c|}{ SOUTH CAROLINA 2011-2015 ACS DATA } \\
\hline Labor force & $3,338,961$ & & Labor force & $3,22,462$ & \\
\hline $\begin{array}{l}\text { Single earner below } \$ 10.21 / \mathrm{hr} \\
(\$ 21,564)\end{array}$ & $1,422,421$ & $42.60 \%$ & $\begin{array}{l}\text { Single earner below } \$ 11.03 / \mathrm{hr} \\
(\$ 23,296)\end{array}$ & $1,587,128$ & $45.06 \%$ \\
\hline $\begin{array}{l}\text { Household low wage threshold } \\
(\$ 43,127)\end{array}$ & $2,522,054$ & $75.53 \%$ & $\begin{array}{l}\text { Household low wage threshold } \\
(\$ 46,591)\end{array}$ & $2,704,159$ & $76.77 \%$ \\
\hline \multicolumn{3}{|c|}{ SOUTH DAKOTA 2006-2010 ACS DATA } & \multicolumn{3}{|c|}{ SOUTH DAKOTA 2011-2015 ACS DATA } \\
\hline Labor force & 655,173 & & Labor force & 695,195 & \\
\hline Single earner below $\$ 9.91 / \mathrm{hr}(\$ 20,930)$ & 273,461 & $41.74 \%$ & $\begin{array}{l}\text { Single earner below } \$ 10.86 / \mathrm{hr} \\
(\$ 22,936)\end{array}$ & 286,716 & $41.24 \%$ \\
\hline $\begin{array}{l}\text { Household low wage threshold } \\
(\$ 41,860)\end{array}$ & 514,606 & $78.55 \%$ & $\begin{array}{l}\text { Household low wage threshold } \\
(\$ 45,873)\end{array}$ & 537,527 & $77.32 \%$ \\
\hline \multicolumn{3}{|c|}{ TENNESSEE 2006-2010 ACS DATA } & \multicolumn{3}{|c|}{ TENNESSEE 2011-2015 ACS DATA } \\
\hline Labor force & $4,620,027$ & & Labor force & $4,846,678$ & \\
\hline $\begin{array}{l}\text { Single earner below } \$ 10.25 / \mathrm{hr} \\
(\$ 21,648)\end{array}$ & $2,013,938$ & $43.59 \%$ & $\begin{array}{l}\text { Single earner below } \$ 11.05 / \mathrm{hr} \\
(\$ 23,338)\end{array}$ & $2,157,813$ & $44.52 \%$ \\
\hline $\begin{array}{l}\text { Household low wage threshold } \\
(\$ 43,296)\end{array}$ & $3,514,032$ & $76.06 \%$ & $\begin{array}{l}\text { Household low wage threshold } \\
(\$ 46,675)\end{array}$ & $3,699,114$ & $76.32 \%$ \\
\hline \multicolumn{3}{|c|}{ TEXAS 2006-2010 ACS DATA } & \multicolumn{3}{|c|}{ TEXAS 2011-2015 ACS DATA } \\
\hline Labor force & $19,292,613$ & & Labor force & $21,520,779$ & \\
\hline $\begin{array}{l}\text { Single earner below } \$ 10.48 / \mathrm{hr} \\
(\$ 22,134)\end{array}$ & $8,647,215$ & $44.82 \%$ & $\begin{array}{l}\text { Single earner below } \$ 11.15 / \mathrm{hr} \\
(\$ 24,549)\end{array}$ & $9,795,780$ & $45.52 \%$ \\
\hline $\begin{array}{l}\text { Household low wage threshold } \\
(\$ 44,268)\end{array}$ & $14,237,258$ & $73.80 \%$ & $\begin{array}{l}\text { Household low wage threshold } \\
(\$ 47,098)\end{array}$ & $15,641,165$ & $72.68 \%$ \\
\hline \multicolumn{3}{|c|}{ UTAH 2006-2010 ACS DATA } & \multicolumn{3}{|c|}{ UTAH 2011-2015 ACS DATA } \\
\hline Labor force & $2,245,118$ & & Labor force & $2,518,783$ & \\
\hline $\begin{array}{l}\text { Single earner below } \$ 13.43 / \mathrm{hr} \\
(\$ 28,364)\end{array}$ & $1,295,028$ & $57.68 \%$ & $\begin{array}{l}\text { Single earner below } \$ 14.28 / \mathrm{hr} \\
(\$ 30,160)\end{array}$ & $1,471,312$ & $58.41 \%$ \\
\hline $\begin{array}{l}\text { Household low wage threshold } \\
(\$ 56,728)\end{array}$ & $1,912,441$ & $85.18 \%$ & $\begin{array}{l}\text { Household low wage threshold } \\
(\$ 60,319)\end{array}$ & $2,121,129$ & $84.21 \%$ \\
\hline \multicolumn{3}{|c|}{ VIRGINIA 2006-2010 ACS DATA } & \multicolumn{3}{|c|}{ VIRGINIA 2011-2015 ACS DATA } \\
\hline Labor force & $6,599,846$ & & Labor force & $7,052,544$ & \\
\hline $\begin{array}{l}\text { Single earner below } \$ 12.32 / \mathrm{hr} \\
(\$ 26,020)\end{array}$ & $2,828,597$ & $42.86 \%$ & $\begin{array}{l}\text { Single earner below } \$ 16.08 / \mathrm{hr} \\
(\$ 33,961)\end{array}$ & $3,714,126$ & $52.66 \%$ \\
\hline $\begin{array}{l}\text { Household low wage threshold } \\
(\$ 52,040)\end{array}$ & $4,843,038$ & $73.38 \%$ & $\begin{array}{l}\text { Household low wage threshold } \\
(\$ 67,922)\end{array}$ & $5,617,479$ & $79.65 \%$ \\
\hline \multicolumn{3}{|c|}{ WASHINGTON 2006-2010 ACS DATA } & \multicolumn{3}{|c|}{ WASHINGTON 2011-2015 ACS DATA } \\
\hline Labor force & $5,234,932$ & & Labor force & $5,661,230$ & \\
\hline $\begin{array}{l}\text { Single earner below } \$ 13.36 / \mathrm{hr} \\
(\$ 28,217)\end{array}$ & $2,459,891$ & $46.99 \%$ & $\begin{array}{l}\text { Single earner below } \$ 14.99 / \mathrm{hr} \\
(\$ 31,659)\end{array}$ & $2,896,078$ & $51.16 \%$ \\
\hline $\begin{array}{l}\text { Household low wage threshold } \\
(\$ 56,433)\end{array}$ & $4,064,871$ & $77.65 \%$ & $\begin{array}{l}\text { Household low wage threshold } \\
(\$ 63,318)\end{array}$ & $4,471,160$ & $78.98 \%$ \\
\hline \multicolumn{3}{|c|}{ WASHINGTON D. C. 2006-2010 ACS DATA } & \multicolumn{3}{|c|}{ WASHINGTON D. C. 2011-2015 ACS DATA } \\
\hline Labor force & 472,035 & & Labor force & 560,220 & \\
\hline $\begin{array}{l}\text { Single earner below } \$ 16.11 / \mathrm{hr} \\
(\$ 34,025)\end{array}$ & 195,424 & $41.40 \%$ & $\begin{array}{l}\text { Single earner below } \$ 17.32 / \mathrm{hr} \\
(\$ 36,580)\end{array}$ & 219,531 & $39.19 \%$ \\
\hline $\begin{array}{l}\text { Household low wage threshold } \\
(\$ 68,049)\end{array}$ & 342,024 & $72.46 \%$ & $\begin{array}{l}\text { Household low wage threshold } \\
(\$ 73,160)\end{array}$ & 392,590 & $70.08 \%$ \\
\hline \multicolumn{3}{|c|}{ WEST VIRGINIA 2006-2010 ACS DATA } & \multicolumn{3}{|c|}{ WEST VIRGINIA 2011-2015 ACS DATA } \\
\hline Labor force & $1,264,605$ & & Labor force & $1,266,845$ & \\
\hline Single earner below $\$ 9.36 / \mathrm{hr}(\$ 19,769)$ & 509,345 & $40.28 \%$ & $\begin{array}{l}\text { Single earner below } \$ 10.08 / \mathrm{hr} \\
(\$ 21,289)\end{array}$ & 515,030 & $40.65 \%$ \\
\hline $\begin{array}{l}\text { Household low wage threshold } \\
(\$ 39,537)\end{array}$ & 904,740 & $71.54 \%$ & $\begin{array}{l}\text { Household low wage threshold } \\
(\$ 42,578)\end{array}$ & 904,926 & $71.43 \%$ \\
\hline
\end{tabular}

(continued)

DOI: 10.17605/OSF.IO/QF4HY 


\section{APPENDIX G}

Family Economic Self-Sufficiency Weighted Average Summaries for 38 States (continued)

\begin{tabular}{|c|c|c|c|c|c|}
\hline \multicolumn{3}{|c|}{ WISCONSIN 2006-2010 ACS DATA } & \multicolumn{3}{|c|}{ WISCONSIN 2011-2015 ACS DATA } \\
\hline Labor force & $4,664,423$ & & Labor force & $4,778,868$ & \\
\hline $\begin{array}{l}\text { Single earner below } \$ 11.73 / \mathrm{hr} \\
(\$ 24,774)\end{array}$ & $2,110,224$ & $45.24 \%$ & $\begin{array}{l}\text { Single earner below } \$ 13.20 / \mathrm{hr} \\
(\$ 27,879)\end{array}$ & $2,336,429$ & $48.89 \%$ \\
\hline $\begin{array}{l}\text { Household low wage threshold } \\
(\$ 49,548)\end{array}$ & $3,625,123$ & $77.72 \%$ & $\begin{array}{l}\text { Household low wage threshold } \\
(\$ 55,757)\end{array}$ & $3,853,645$ & $80.64 \%$ \\
\hline \multicolumn{3}{|c|}{ WYOMING 2006-2010 ACS DATA } & \multicolumn{3}{|c|}{ WYOMING 2011-2015 ACS DATA } \\
\hline Labor force & 467,635 & & Labor force & 487,316 & \\
\hline $\begin{array}{l}\text { Single earner below } \$ 10.46 / \mathrm{hr} \\
(\$ 22,092)\end{array}$ & 188,348 & $40.28 \%$ & $\begin{array}{l}\text { Single earner below } \$ 12.01 / \mathrm{hr} \\
(\$ 25,365)\end{array}$ & 208,668 & $42.82 \%$ \\
\hline $\begin{array}{l}\text { Household low wage threshold } \\
(\$ 44,183)\end{array}$ & 330,263 & $70.62 \%$ & $\begin{array}{l}\text { Household low wage threshold } \\
(\$ 50,730)\end{array}$ & 345,222 & $70.84 \%$ \\
\hline
\end{tabular}

Source: FESS values retrieved from reports published by Center for Women's Welfare at the University of Washington, at http://www.selfsufficiencystandard.org/state-data. Where needed, values were updated using the CPI Inflation calculator at https://data.bls.gov/cgi-bin/cpicalc.pl. Income data extracted from IPUMS USA database (https://usa.ipums.org). 


\section{APPENDIX H}

\section{Longitudinal Employer-Household Dynamics}

\begin{tabular}{|c|c|c|c|c|}
\hline INDUSTRY & INDUSTRY LABEL VALUE & YEAR & QUARTER & EMPLOYEES \\
\hline 11 & Agriculture, Forestry, Fishing and Hunting & 2001 & 2 & 2,442 \\
\hline 21 & Mining, Quarrying, and Oil and Gas Extraction & 2001 & 2 & 1,071 \\
\hline 22 & Utilities & 2001 & 2 & 2,476 \\
\hline 23 & Construction & 2001 & 2 & 15,982 \\
\hline $31-33$ & Manufacturing & 2001 & 2 & 42,513 \\
\hline 42 & Wholesale Trade & 2001 & 2 & 17,831 \\
\hline $44-45$ & Retail Trade & 2001 & 2 & 48,549 \\
\hline $48-49$ & Transportation and Warehousing & 2001 & 2 & 9,525 \\
\hline 51 & Information & 2001 & 2 & 7,050 \\
\hline 52 & Finance and Insurance & 2001 & 2 & 23,990 \\
\hline 53 & Real Estate and Rental and Leasing & 2001 & 2 & 3,913 \\
\hline 54 & Professional, Scientific, and Technical Services & 2001 & 2 & 8,642 \\
\hline 55 & Management of Companies and Enterprises & 2001 & 2 & 4,392 \\
\hline 56 & $\begin{array}{l}\text { Administrative and Support and Waste } \\
\text { Management and Remediation Services }\end{array}$ & 2001 & 2 & 12,977 \\
\hline 61 & Educational Services & 2001 & 2 & 35,296 \\
\hline 62 & Health Care and Social Assistance & 2001 & 2 & 48,973 \\
\hline 71 & Arts, Entertainment, and Recreation & 2001 & 2 & 6,640 \\
\hline 72 & Accommodation and Food Services & 2001 & 2 & 30,835 \\
\hline 81 & Other Services (except Public Administration) & 2001 & 2 & 10,469 \\
\hline \multirow[t]{2}{*}{92} & Public Administration & 2001 & 2 & 19,033 \\
\hline & & $\begin{array}{l}2001 \\
\text { Total }\end{array}$ & & 352,599 \\
\hline 11 & Agriculture, Forestry, Fishing and Hunting & 2006 & 2 & 3,271 \\
\hline 21 & Mining, Quarrying, and Oil and Gas Extraction & 2006 & 2 & 666 \\
\hline 22 & Utilities & 2006 & 2 & 2,391 \\
\hline 23 & Construction & 2006 & 2 & 20,579 \\
\hline $31-33$ & Manufacturing & 2006 & 2 & 41,101 \\
\hline 42 & Wholesale Trade & 2006 & 2 & 18,633 \\
\hline $44-45$ & Retail Trade & 2006 & 2 & 48,624 \\
\hline $48-49$ & Transportation and Warehousing & 2006 & 2 & 9,615 \\
\hline 51 & Information & 2006 & 2 & 7,074 \\
\hline 52 & Finance and Insurance & 2006 & 2 & 24,457 \\
\hline 53 & Real Estate and Rental and Leasing & 2006 & 2 & 4,075 \\
\hline
\end{tabular}

(continued) 


\section{APPENDIX $\mathrm{H}$}

Longitudinal Employer-Household Dynamics (continued)

\begin{tabular}{|c|c|c|c|c|}
\hline INDUSTRY & INDUSTRY LABEL VALUE & YEAR & QUARTER & EMPLOYEES \\
\hline 54 & Professional, Scientific, and Technical Services & 2006 & 2 & 10,357 \\
\hline 55 & Management of Companies and Enterprises & 2006 & 2 & 2,929 \\
\hline 56 & $\begin{array}{l}\text { Administrative and Support and Waste } \\
\text { Management and Remediation Services }\end{array}$ & 2006 & 2 & 13,125 \\
\hline 61 & Educational Services & 2006 & 2 & 34,294 \\
\hline 62 & Health Care and Social Assistance & 2006 & 2 & 53,010 \\
\hline 71 & Arts, Entertainment, and Recreation & 2006 & 2 & 7,434 \\
\hline 72 & Accommodation and Food Services & 2006 & 2 & 33,705 \\
\hline 81 & Other Services (except Public Administration) & 2006 & 2 & 10,142 \\
\hline \multirow[t]{2}{*}{92} & Public Administration & 2006 & 2 & 19,463 \\
\hline & & 2006 Total & & 364,945 \\
\hline 11 & Agriculture, Forestry, Fishing and Hunting & 2011 & 2 & 4,246 \\
\hline 21 & Mining, Quarrying, and Oil and Gas Extraction & 2011 & 2 & 752 \\
\hline 22 & Utilities & 2011 & 2 & 2,429 \\
\hline 23 & Construction & 2011 & 2 & 18,742 \\
\hline $31-33$ & Manufacturing & 2011 & 2 & 38,668 \\
\hline 42 & Wholesale Trade & 2011 & 2 & 19,058 \\
\hline $44-45$ & Retail Trade & 2011 & 2 & 49,944 \\
\hline $48-49$ & Transportation and Warehousing & 2011 & 2 & 9,513 \\
\hline 51 & Information & 2011 & 2 & 6,528 \\
\hline 52 & Finance and Insurance & 2011 & 2 & 23,664 \\
\hline 53 & Real Estate and Rental and Leasing & 2011 & 2 & 3,725 \\
\hline 54 & Professional, Scientific, and Technical Services & 2011 & 2 & 11,908 \\
\hline 55 & Management of Companies and Enterprises & 2011 & 2 & 4,133 \\
\hline 56 & $\begin{array}{l}\text { Administrative and Support and Waste } \\
\text { Management and Remediation Services }\end{array}$ & 2011 & 2 & 13,095 \\
\hline 61 & Educational Services & 2011 & 2 & 37,475 \\
\hline 62 & Health Care and Social Assistance & 2011 & 2 & 59,287 \\
\hline 71 & Arts, Entertainment, and Recreation & 2011 & 2 & 7,229 \\
\hline 72 & Accommodation and Food Services & 2011 & 2 & 35,486 \\
\hline 81 & Other Services (except Public Administration) & 2011 & 2 & 9,969 \\
\hline \multirow[t]{2}{*}{92} & Public Administration & 2011 & 2 & 20,549 \\
\hline & & 2011 Total & & 376,400 \\
\hline
\end{tabular}

DOI: 10.17605/OSF.IO/QF4HY 


\section{APPENDIX H}

Longitudinal Employer-Household Dynamics (continued)

\begin{tabular}{|c|l|c|r|r|}
\hline INDUSTRY & INDUSTRY LABEL VALUE & YEAR & QUARTER & EMPLOYEES \\
\hline 11 & Agriculture, Forestry, Fishing and Hunting & 2016 & 2 & 5,501 \\
\hline 21 & Mining, Quarrying, and Oil and Gas Extraction & 2016 & 2 & 805 \\
\hline 22 & Utilities & 2016 & 2 & 2,331 \\
\hline 23 & Construction & 2016 & 2 & 21,677 \\
\hline $31-33$ & Manufacturing & 2016 & 2 & 42,105 \\
\hline 42 & Wholesale Trade & 2016 & 2 & 21,444 \\
\hline $44-45$ & Retail Trade & 2016 & 2 & 53,467 \\
\hline $48-49$ & Transportation and Warehousing & 2016 & 2 & 10,566 \\
\hline 51 & Information & 2016 & 2 & 5,893 \\
\hline 52 & Finance and Insurance & 2016 & 2 & 24,286 \\
\hline 53 & Real Estate and Rental and Leasing & 2016 & 2 & 4,137 \\
\hline 54 & Professional, Scientific, and Technical Services & 2016 & 2 & 13,582 \\
\hline 55 & Management of Companies and Enterprises & 2016 & 2 & 5,452 \\
\hline 56 & Administrative and Support and Waste & 2016 & 2 & 13,089 \\
\hline 61 & Management and Remediation Services & 2016 & 2 & 38,277 \\
\hline 62 & Educational Services & 2016 & 2 & 63,593 \\
\hline 71 & Health Care and Social Assistance & 2016 & 2 & 7,436 \\
\hline 72 & Arts, Entertainment, and Recreation & 2016 & 2 & 38,943 \\
\hline 81 & Accommodation and Food Services & 2016 & 2 & 10,747 \\
\hline 92 & Other Services (except Public Administration) & 2016 & 2 & 21,458 \\
\hline & Public Administration & $\mathbf{2 0 1 6}$ Total & & $\mathbf{4 0 4 , 7 8 9}$ \\
\hline & & Grand Total & & $1,498,733$ \\
\hline
\end{tabular}

Source: Longitudinal Employer-Household Dynamics (LEHD, 2017). Quarterly workforce indicators (QWI) data extraction, limited to quarter 2 in years 2001, 2006, 2011, and 2016. U. S. Census Bureau. Retrieved from https://lehd.ces.census.gov. Reformatted for confined area. 